Florida International University FIU Digital Commons

$3-2-2012$

\title{
Microteaching Lesson Study: Mentor Interaction Structure and its Relation to Elementary Preservice Mathematics Teacher Knowledge Development
}

Roxanne Molina

Florida International University, rmoli002@fiu.edu

DOI: $10.25148 /$ etd.FI12050222

Follow this and additional works at: https://digitalcommons.fiu.edu/etd

\section{Recommended Citation}

Molina, Roxanne, "Microteaching Lesson Study: Mentor Interaction Structure and its Relation to Elementary Preservice Mathematics Teacher Knowledge Development" (2012). FIU Electronic Theses and Dissertations. 616.

https://digitalcommons.fiu.edu/etd/616 


\title{
FLORIDA INTERNATIONAL UNIVERSITY
}

Miami, Florida

\section{MICROTEACHING LESSON STUDY: MENTOR INTERACTION STRUCTURE AND ITS RELATION TO ELEMENTARY PRESERVICE MATHEMATICS TEACHER KNOWLEDGE DEVELOPMENT}

\author{
A dissertation submitted in partial fulfillment of the \\ requirements for the degree of \\ DOCTOR OF PHILOSOPHY \\ in \\ CURRICULUM AND INSTRUCTION \\ by \\ Roxanne V. Molina
}


To: Dean Delia C. Garcia

College of Education

This dissertation, written by Roxanne V. Molina, and entitled Microteaching Lesson Study: Mentor Interaction Structure and its Relation to Elementary Preservice Mathematics Teacher Knowledge Development, having been approved in respect to style and intellectual content, is referred to you for judgment.

We have read this dissertation and recommend that it be approved.

Laura H. Dinehart

George E. O’Brien

Joan Wynne

Maria L. Fernandez, Major Professor

Date of Defense: March 2, 2012

The dissertation of Roxanne V. Molina is approved.

Dean Delia C. Garcia

College of Education

Dean Lakshmi N. Reddi

University Graduate School

Florida International University, 2012 
(C) Copyright 2012 by Roxanne V. Molina All rights reserved. 


\section{DEDICATION}

I dedicate this dissertation to my husband, whose love and support was a key component in my success and to my daughter, who was patient and understanding during this lengthy process. 


\section{ACKNOWLEDGMENTS}

I wish to thank the members of my committee, Dr. Fernandez, Dr. Dinehart, Dr. O’Brien, and Dr. Wynne, for their support and guidance through this process. In particular, Dr.

Fernandez, who spent a substantial amount of time helping me to attain a doctoral degree. In addition, I would like to thank my friends and family members who helped in different ways, so that I could complete this dissertation. 


\author{
ABSTRACT OF THE DISSERTATION \\ MICROTEACHING LESSON STUDY: MENTOR INTERACTION STRUCTURE \\ AND ITS RELATION TO ELEMENTARY PRESERVICE \\ MATHEMATICS TEACHER KNOWLEDGE DEVELOPMENT
}

by

Roxanne V. Molina

Florida International University, 2012

Miami, Florida

\title{
Professor Maria L. Fernandez, Major Professor
}

This study investigated Microteaching Lesson Study (MLS) and three possible MLS mentor interaction structures during the debriefing sessions in relation to elementary preservice teacher development of knowledge for teaching. One hundred three elementary preservice teachers enrolled in five different sections of a mathematics methods course at a southern urban university were part of the study. This included 72 participants who completed MLS across three different mentor interaction structures as part of their course requirements and 31 elementary preservice teachers who did not complete MLS as part of their methods course and served as a comparison group for a portion of the study. A sequential mixed-methods research design was used to analyze the relationship between MLS mentor interaction structure and growth in preservice teachers' mathematics teacher knowledge. Data sources included pre and post assessments, group developed lesson plans and final reports, a feedback survey with Likerttype and open-ended questions, and transcripts of audio-recorded debriefing sessions. The pre and post assessments were analyzed using Analysis of Variance (ANOVA) and descriptive statistics were used to analyze the Likert-type feedback survey questions. Group MLS lesson plans, final reports, and transcripts of debriefing sessions along with the open-ended questions 
from the feedback survey were coded in a three-step process as described by Miles and Huberman (1994).

In alignment with findings from M. Fernandez (2005, 2010), elementary presrvice teachers participating in MLS grew in content knowledge related to MLS topics taught by one another. Results from the analysis of pre and post content knowledge assessments revealed that participants grew in their understanding of the mathematics topics taught during MLS irrespective of their mentor interaction structure and when compared to the participants who did not complete MLS in their methods course. Findings from the analysis of lesson plans for growth in pedagogical content knowledge revealed the most growth in this area occurred for participants assigned to the interaction structure in which the MLS mentor participated in the first two debriefing sessions. Analysis of the transcripts of the discourse during the debriefing sessions and the feedback surveys support the finding that the elementary preservice teachers assigned to the interaction structure in which the MLS mentor participated in the first and second debriefing sessions benefited more from the MLS experience when compared to elementary preservice teachers assigned to the other two interaction structures (MLS mentor participated in only the first debriefing session and MLS mentor participated in only the last debriefing session). 


\section{TABLE OF CONTENTS}

CHAPTER

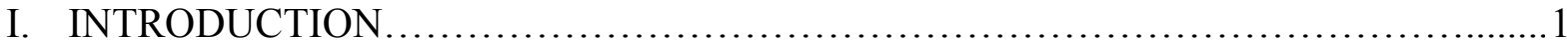

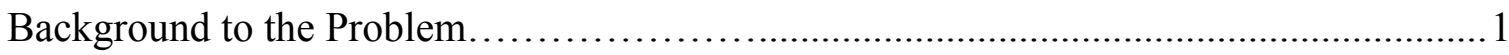

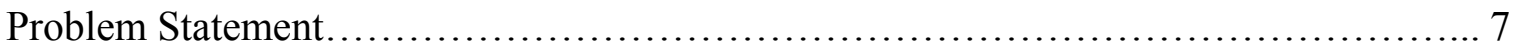

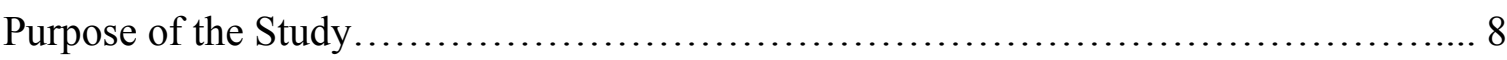

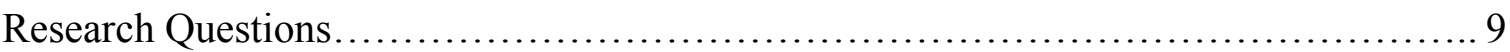

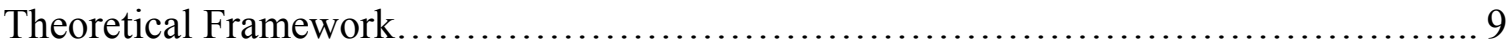

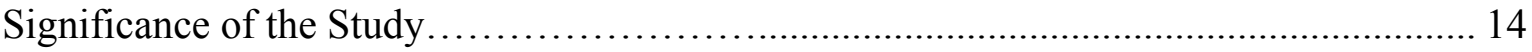

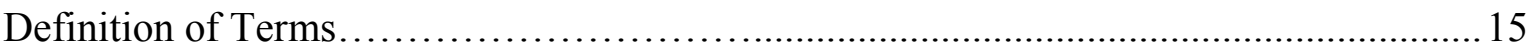

Assumptions and Delimitations of the Study ...................................... 15

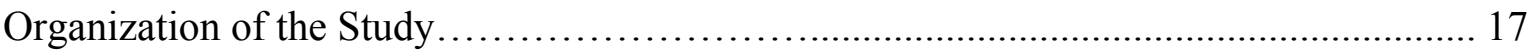

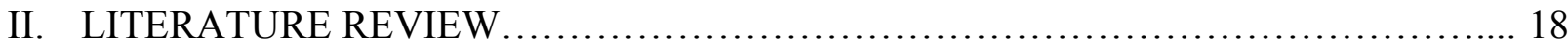

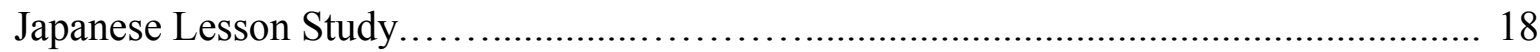

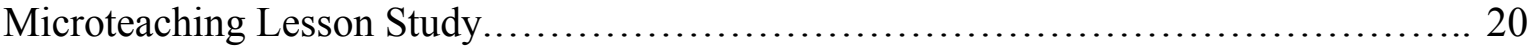

Research Studies Involving Microteaching Lesson Study ............................. 21

Lesson Study with Preservice Teachers........................................... 26

Lesson Study with Practicing Teachers..............................................34

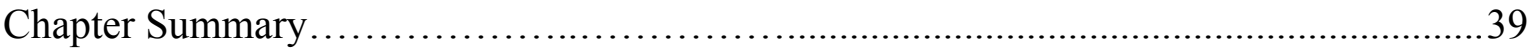

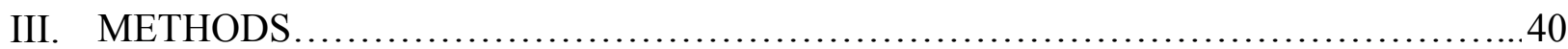

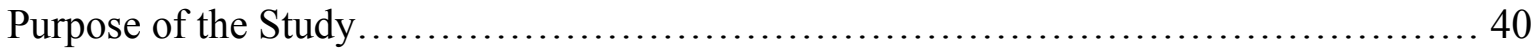

Research Questions and Hypothesis................................................ 40

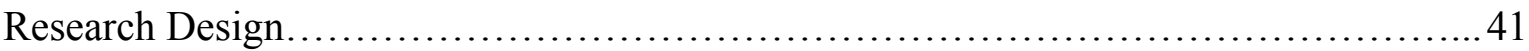

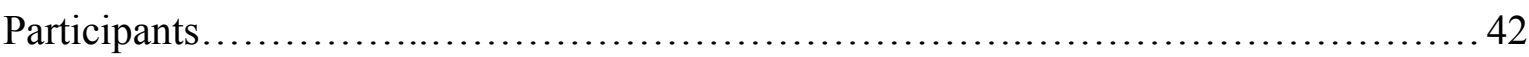

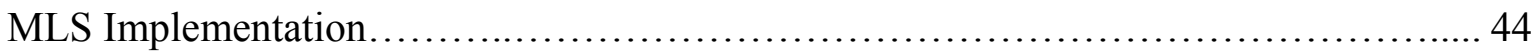

Data Collection and Analysis...................................................... 49

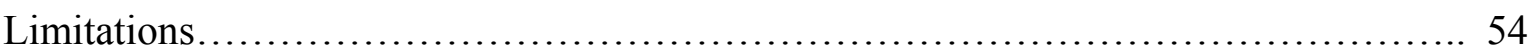

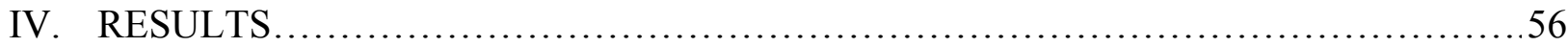

Mathematical Content Knowledge Analysis..........................................56

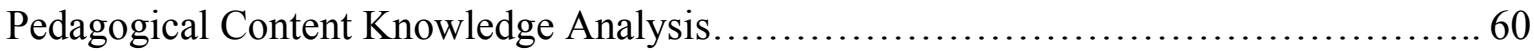

Big Idea \#1: Understanding students' current understanding.........................6 60

Big Idea \#2: Students knowing in one way do not necessarily know in the other(s)... 62

Big Idea \#3: Intuitive knowledge is both an asset and a liability...................... 65

Big idea \#4: Certain instructional characteristics promote retention.................. 67 
Big idea \#5: Alternative representations and the recognition and analysis of alternative methods are important............................................ 70

Discourse Analysis...................................................... 72

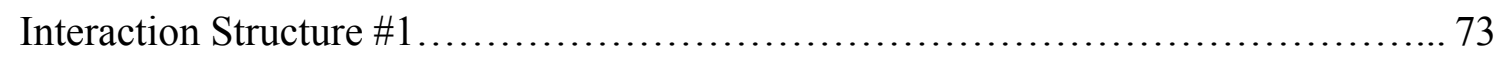

Groups with Highest Gains............................................... 73

Groups with Least Gains................................................. 79

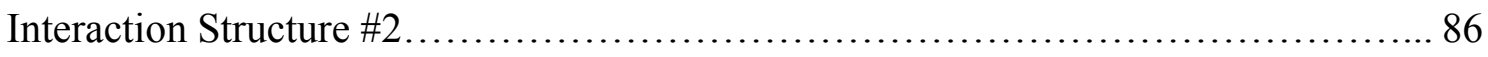

Groups with Highest Gains.............................................. 87

Groups with Least Gains................................................ 93

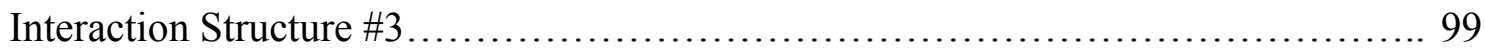

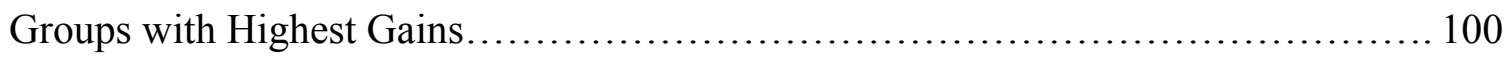

Groups with Least Gains................................................ 109

Feedback Survey ...................................................... 112

V. DISCUSSION, RECOMMENDATIONS AND CONCLUSIONS....................... 118

Summary of Findings....................................................... 118

Discussion of the Findings................................................ 120

Growth in Content Knowledge as a consequence of participation in MLS.............. 120

Richer PCK discussions with MLS mentor ..................................... 122

Value of formative vs. summative interactions with MLS mentor....................... 126

Insights related to Graeber's (1999) five big ideas................................. 127

Recommendations for Future Research........................................ 128

Conclusions.............................................................. 129

REFERENCES .............................................................. 130

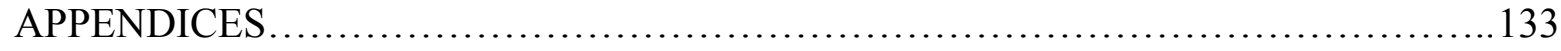

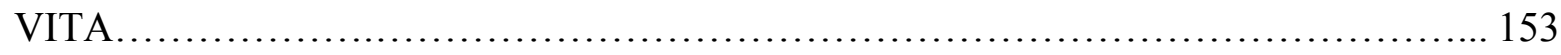




\section{LIST OF TABLES}

TABLE

1. Example Schedule for MLS Implementation................................ 47

2. Groups per Interaction Structure ........................................ 48

3. Average Weighted Scores for Initial Pre and Post Assessment for MLS and nonMLS groups........................................................ 58

4. Average Weighted Scores as Percents for Second Pre and Post Assessment......... 59

5. Percentage of Low, Medium, and High Lessons per Interaction Structure for Big Idea \#1: Understanding students' current understanding ....................... 61

6. Percentage of Low, Medium, and High Lessons per Interaction Structure for Big Idea \#2: Students knowing in one way do not necessarily know in the other(s).....66

7. Percentage of Low, Medium, and High Lessons per Interaction Structure for Big Idea \#3: Intuitive knowledge is both an asset and a liability.

8. Percentage of Low, Medium, and High Lessons per Interaction Structure for Big Idea \#4: Certain instructional characteristics appear to promote retention........... 68

9. Percentage of Low, Medium, and High Lessons per Interaction Structure for Big Idea \#5: Alternative representations and the recognition and analysis of alternative methods are important.

10. Percentage of words per theme for debriefing sessions with Properties of Quadrilaterals (Course Section \#2) group.

11. Percentage of words per Graeber's (1999) Big Ideas for Pedagogical Content Knowledge Discussions theme with Properties of Quadrilaterals (Course Section

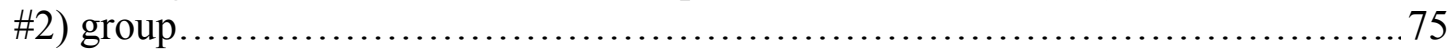

12. Percentage of words per theme for debriefing sessions with Properties of Quadrilaterals (Course Section \#1) group.

13. Percentage of words per Graeber's (1999) Big Ideas for Pedagogical Content Knowledge Discussions theme with Properties of Quadrilaterals (Course Section

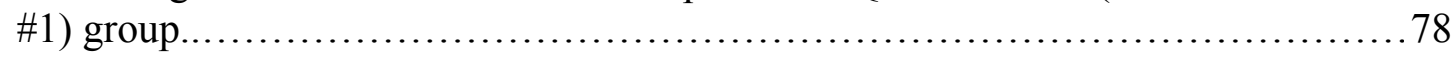

14. Percentage of words per theme for debriefing sessions with Adding Fractions (Course Section \#1) group.

15. Percentage of words per Graeber's (1999) Big Ideas for Pedagogical Content 
Knowledge Discussions theme for Adding Fractions (Course Section \#1) group...... 81

16. Percentage of words per theme for debriefing sessions with Adding Fractions (Course Section \#2) group............................................. 83

17. Percentage of words per Graeber's (1999) Big Ideas for Pedagogical Content Knowledge Discussions theme for Adding Fractions (Course Section \#2) group...... 84

18. Percentage of words per theme for debriefing sessions with Multiplying Fractions (Course Section \#2) group.

19. Percentage of words per Graeber's (1999) Big Ideas for Pedagogical Content Knowledge Discussions theme for Multiplying Fractions (Course Section \#2) group.

20. Percentage of words per theme for debriefing sessions with Fractions, Decimals, and Percents (Course Section \#3) group.................................... 90

21. Percentage of words per Graeber's (1999) Big Ideas for Pedagogical Content Knowledge Discussions theme for Fractions, Decimals, and Percents (Course Section \#3) group. 92

22. Percentage of words per theme for debriefing sessions with Multiplying Fractions (Course Section \#1) group.

23. Percentage of words per Graeber's (1999) Big Ideas for Pedagogical Content Knowledge Discussions theme for Fractions, Decimals, and Percents (Course Section \#1) group.

24. Percentage of words per theme for debriefing sessions with Addition of two-digit numbers (Course Section \#1) group..................................... 96

25. Percentage of words per Graeber's (1999) Big Ideas for Pedagogical Content Knowledge Discussions theme for Addition of two-digit numbers (Course Section

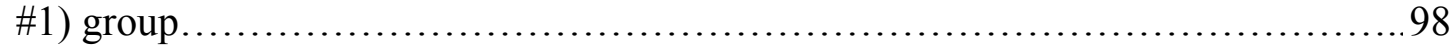

26. Percentage of words per theme for debriefing sessions with Properties of Quadrilaterals (Course Section \#2) group.

27. Percentage of words per Graeber's (1999) Big Ideas for Pedagogical Content Knowledge Discussions theme with Area Formulas (Course Section \#2) group...... 102

28. Percentage of words per theme for debriefing sessions with Area Formulas (Course Section \#3) group. 103

29. Percentage of words per Graeber's (1999) Big Ideas for Pedagogical Content 
Knowledge Discussions theme for Area Formulas (Course Section \#3) group

30. Percentage of words per theme for debriefing sessions with Comparing Fractions (Course Section \#1) group................................................. 106

31. Percentage of words per Graeber's (1999) Big Ideas for Pedagogical Content Knowledge Discussions theme for Adding Fractions (Course Section \#1) group...... 108

32. Percentage of words per theme for debriefing sessions for Developing Equations (Course Section \#1) group.................................................. 109

33. Percentage of words per Graeber's (1999) Big Ideas for Pedagogical Content Knowledge Discussions theme for Developing Equations (Course Section \#1)

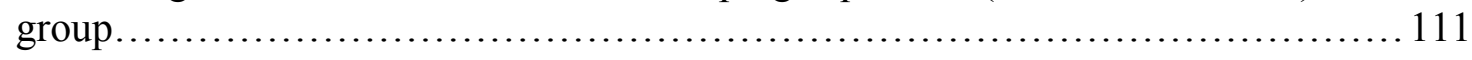

34. Average Response and Standard Deviations to Likert-type Statements on Feedback

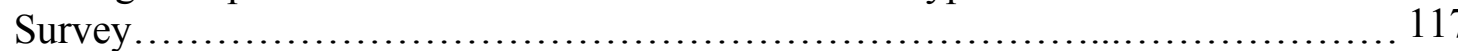




\section{LIST OF FIGURES}

FIGURE

1. Graph of Growth in Graeber's Big Idea \#1: Understanding students' current understanding per interaction structure.

2. Graph of Growth in Graeber's Big Idea \#2: Students knowing in one way do not necessarily know in the other(s).

3. Graph of Growth in Graeber's Big Idea \#3: Intuitive knowledge is both an asset and a liability.....

4. Graph of Growth in Graeber's Big Idea \#4: Certain instructional characteristics appear to promote retention.

5. Graph of Growth in Graeber's Big Idea \#5: Alternative representations and the recognition and analysis of alternative methods are important.

6. Bar graph of percent of words per theme for each debriefing session for Properties of Quadrilaterals (Course Section \#2) group.

7. Bar graph of percent of words per Graeber's (1999) Big Idea for Pedagogical Content Knowledge Discussions theme for Properties of Quadrilaterals (Course Section \#2) group.

8. Bar graph of percent of words per theme for each debriefing session for Properties of Quadrilaterals (Course Section \#1) group.

9. Bar graph of percent of words per Graeber's (1999) Big Ideas for Pedagogical Content Knowledge Discussions theme for Properties of Quadrilaterals (Course Section \#1) group.

10. Bar graph of percent of words per theme for each debriefing session for Adding Fractions (Course Section \#1) group.

11. Bar graph of percent of words per Graeber's (1999) Big Ideas for Pedagogical Content Knowledge Discussions theme for Adding Fractions (Course Section \#1) group.

12. Bar graph of percent of words per theme for each debriefing session for Adding Fractions (Course Section \#2) group

13. Bar graph of percent of words per Graeber's (1999) Big Ideas for Pedagogical Content Knowledge Discussions theme for Adding Fractions (Course Section \#2) group. 
14. Bar graph of percent of words per theme for each debriefing session for Multiplying Fractions (Course Section \#2) group.

15. Bar graph of percent of words per Graeber's (1999) Big Ideas for Pedagogical Content Knowledge Discussions theme for Multiplying Fractions (Course Section \#2) group.

16. Bar graph of percent of words per theme for each debriefing session for Fractions, Decimals, and Percents (Course Section \#3) group.

17. Bar graph of percent of words per Graeber's (1999) Big Ideas for Pedagogical Content Knowledge Discussions theme for Fractions, Decimals, and Percents (Course Section \#3) group.

18. Bar graph of percent of words per theme for each debriefing session for Multiplying Fractions (Course Section \#1) group.

19. Bar graph of percent of words per Graeber's (1999) Big Ideas for Pedagogical Content Knowledge Discussions theme for Fractions, Decimals, and Percents (Course Section \#3) group.

20. Bar graph of percent of words per theme for each debriefing session for Addition of two-digit numbers (Course Section \#1) group.................................... 97

21. Bar graph of percent of words per Graeber's (1999) Big Ideas for Pedagogical Content Knowledge Discussions theme for Addition of two-digit numbers (Course Section \#3) group ............................................................. 98

22. Bar graph of percent of words per theme for each debriefing session for Properties of Quadrilaterals (Course Section \#2) group.

23. Bar graph of percent of words per Graeber's (1999) Big Idea for Pedagogical Content Knowledge Discussions theme for Area Formulas (Course Section \#2) group. 102

24. Bar graph of percent of words per theme for each debriefing session for Area Formulas (Course Section \#3) group.

25. Bar graph of percent of words per Graeber's (1999) Big Ideas for Pedagogical Content Knowledge Discussions theme for Area Formulas (Course Section \#3) group.

26. Bar graph of percent of words per theme for each debriefing session for Adding Fractions (Course Section \#1) group. 
27. Bar graph of percent of words per Graeber's (1999) Big Ideas for Pedagogical Content Knowledge Discussions theme for Comparing Fractions (Course Section \#1) group ..................................................................... 108

28. Bar graph of percent of words per theme for each debriefing session for Developing Equations (Course Section \#1) group...................................... 110

29. Bar graph of percent of words per Graeber's (1999) Big Ideas for Pedagogical Content Knowledge Discussions theme for Developing Equations (Course Section \#1) group 


\section{Chapter I}

\section{Introduction}

\section{Background to the Problem}

When compared internationally to their peers, United States' students do not come close to the level of achievement reached by their peers (National Mathematics Advisory Panel (NMAP), 2008). The 2007 Trends in International Mathematics and Science Study (TIMSS) found for fourth grade, United States students ranked eleventh for mathematics achievement when compared with students from other countries. The top performing countries for fourth grade were in rank order: Hong Kong, Singapore, China, Japan, and Kazakhstan. For eighth grade, United States students ranked ninth for mathematics achievement with China, Korea, Singapore, Hong Kong, and Japan performing the best.

Results from national comparisons of student mathematics achievement are equally startling. The National Mathematics Advisory Panel (NMAP) report (2008) indicated that $32 \%$ of U.S. students are at or above the "proficient" level in Grade 8 and 23\% at Grade 12. One of the panel's major conclusions was that the "delivery system in mathematics education- the system that translates mathematics knowledge into value and ability for the next generation- is broken and must be fixed" (NMAP, 2008, p. xii). As part of its recommendations for improving K-12 mathematics education, the NMAP report, suggests recognizing mathematically knowledgeable classroom teachers as having a central role in mathematics education

Teacher education is cited as an area in need of reform by the TIMSS National Center for Education Statistics (NCES) 1999 report, which is based on an international assessment of fourth and eighth graders' performance in mathematics (Menon, 2000). The TIMSS (NCES,1999) report is only one of numerous reports in the last two decades acknowledging that 
the lack of teacher preparation in the United States, particularly in the areas of mathematics and science, has contributed to below average student performance in these subjects when compared internationally (Darling-Hammond, 2001). The answer to improving student mathematics achievement, therefore, "lies in envisioning a more systematic change that takes into consideration both the mores of the culture and the preparation of mathematics teachers" (Menon, 2000). This suggests a necessary component in improving student mathematics achievement is appropriately prepared teachers.

While multiple factors influence teacher preparation, one important component is the quality of the courses required for completion of a teaching degree. Several studies have noted the importance of engaging preservice teachers in authentic practice oriented experiences to help develop their mathematics knowledge (C. Fernandez et al., 2003; Graeber, 1999; Parks, 2007). For example, Graeber notes, "I have found that only by combining reading about common misconceptions and limited conceptions with interviewing or tutoring experiences are preservice teachers convinced of the need to understand students' understanding" (Graeber, 1999, p. 194). This suggests a need to design courses that engage preservice teachers in practice-based experiences that help deepen their mathematics knowledge and knowledge of others' mathematics understanding to better prepare them to work with students.

Given the success of students in mathematics in other countries, one possibility to improve the quality of mathematics methods courses offered at the university level is to investigate approaches to teacher preparation and professional development of countries with high-achieving mathematics students. One strategy that stands out as an example of a best practice amongst countries with high-achieving mathematics students is the use of lesson study as a professional development process for teachers in Japan (Stigler \& Hiebert, 1999). Lesson study, typically, 
involves a team of three to five teachers who work in conjunction with an outside specialist to develop a "research lesson," a lesson that is used to test teacher ideas about a particular topic (Stigler \& Hiebert, 1999; Takahashi \& Yoshida, 2004). Group members work collaboratively to create a lesson based on an overarching student learning goal that may be assigned at the administrative level or developed by the group. Then, one of the group members teaches the lesson as the other members along with the outside specialist observe. Afterwards all group members and the outside specialist meet in what is known as a debriefing session to discuss the lesson. Based on the debriefing session, the group will then revise the lesson and another member teaches the new lesson. This cycle of lesson planning, presentation, debriefing, and revision will continue until the group is satisfied with the results of the lesson. Through the collaborative revision of the research lesson, teachers develop subject matter and pedagogical knowledge (M. Fernandez, 2010; C. Fernandez et al., 2003; Lewis et al., 2006; Lewis et al., 2004; McMahon \& Hines, 2008.).

Lesson study began to emerge in the United States following its introduction in The Teaching Gap (Stigler \& Hiebert, 1999). Within four years of its introduction, lesson study had spread to more than 335 United States schools spanning 32 states (Lewis et al., 2006; Takahasi \& Yoshida, 2004). Adaptations of the process have also emerged. For example, at the university level instructors have modified the process for use with preservice teachers. While various modifications have arisen, of particular interest because of its success with secondary mathematics preservice teachers, is Microteaching Lesson Study (MLS) (M. Fernandez, 2005, 2010).

Similar to lesson study, MLS engages preservice teachers in cycles of lesson development, implementation and revision of an assigned topic while teaching to small groups of 
their peers in their university classroom or small groups of K-12 students (M. Fernandez, 2005, 2006, 2010). Preservice teachers work in groups of at most three in conjunction with a MLS mentor. The MLS mentor is similar to the outside specialist in lesson study in that the individual is knowledgeable in the lesson content, content area teaching, and lesson study. The role may be assumed by the course instructor, curriculum specialist or field placement supervisor. As in lesson study, the topic is purposefully selected with an overarching student learning goal in mind. Aspects of MLS taken from microteaching include teaching small groups of peers or small groups of K-12 students, teaching a somewhat shortened lesson (approximately 25 to 30 minutes) and the use of video to capture teacher lessons for later analysis (which is optional in microteaching but requisite in MLS).

MLS research, research involving other variations of lesson study with preservice teachers, and research with practicing teachers over the last decade all indicate the effectiveness of U.S. lesson study variations for teacher growth and development in both content and pedagogical knowledge (C. Fernandez et al., 2003; M. Fernandez, 2005, 2006, \& 2010; Lewis et al., 2006; Lewis et al., 2004; McMahon \& Hines, 2008). Researchers report positive findings indicating the benefits associated with the professional development process are substantial. These benefits include improved content knowledge for teachers, enhancement of teacher pedagogy, higher teacher self-efficacy and motivation, development of teacher ability to observe and focus on student learning, improved teacher reflection ability, and creation of collaborative networks for teachers (C. Fernandez et al., 2003; M. Fernandez, 2005, 2006, 2010; Lewis et al., 2004; Lewis et al., 2006; Parks, 2007; Puchner \& Taylor, 2006; Taylor et al., 2005).

However, no study reviewed has investigated to what extent the outside specialist or MLS mentor is a factor in ensuring that participants are able to benefit from the experience of 
lesson study or its variations. Current lesson study and MLS research has focused on examining the outcome of the technique without a thorough examination of the mechanisms that led to the results (C. Fernandez et al., 2003; Lewis et al., 2006). This is a concern as many U.S. educational practices that initially show promise gradually disappear and are replaced with the more current reform model of the time, a phenomenon that has become known as "faddism" (Burkhardt \& Schoenfeld, 2003; Paige, 2002). One explanation for faddism is a lack of sufficient research that effectively examines the mechanisms of the practice, knowledge necessary if positive results are to be attained by any school or district interested in implementing lesson study.

A primary responsibility of the MLS mentor is to engage participants in debriefing sessions. In its current form, the MLS mentor may interact with individual groups and engage them in reflecting and revising the research lesson during each debriefing session. This type of interaction structure allows the mentor to provide support to individual MLS groups throughout the development, analysis, and revision stages of the lesson. It is possible that this type of formative analysis and feedback is a key element in participants' learning through MLS (M. Fernandez, 2010; M. Fernandez \& Zilliox, 2011) and the outcome of the process. However, in the case in which MLS is used in a semester course, the debriefing sessions can occupy the same amount of time required of the lesson implementations. Thus, mentor participation in every debriefing session may become an issue for course instructors who will then need to balance allocating sufficient time for MLS with covering the remainder of their curriculum. Equivalently, if MLS is used with practicing teachers, the role of the mentor may be filled by a curriculum specialist or a university educator who would also experience issues related to time commitment. The number of groups for which a curriculum specialist acts as a mentor would 
need to be balanced by other job responsibilities. In the case of a university educator acting as a mentor for practicing teachers, the issue of meeting time is compounded by driving time to and from the designated meeting locations.

No study reviewed reported how much time the outside specialist in lesson study or the mentor in MLS spent working with a group during the lesson study or MLS process. The amount of time required of the outside specialist or a MLS mentor is a concern for both schools and universities interested in implementing the technique in their respective settings. Interested schools would benefit from this knowledge as they plan for lesson study implementation. Universities may be particularly interested in the time required of a mentor as the course instructor will often assume this role and will likely have other time consuming responsibilities already established.

It is also possible that the discourse that takes place during the debriefing sessions is an important factor in participant learning and the outcomes of the process. The MLS mentor, who is typically either a mathematics educator or curriculum specialist, may be involved in some or all of the debriefing sessions. Differences may exist between the discourse that takes place during the debriefing sessions in which the MLS mentor is present and not present. The MLS mentor may engage the preservice teachers in more mathematically valuable conversations, which may be more helpful early in the process as the MLS mentor would be able to help resolve mathematical misconceptions before students are affected by such errors. It may also be that the MLS mentor's participation in the first debriefing session may be useful in resolving group collaboration issues.

Another possibility is differences may exist amongst the discourse of different MLS mentors. For example, a MLS mentor who engages groups in discussions that challenge their 
ideas through skillfully posed questions may be more helpful to the development of elementary preservice teachers' content and pedagogical content knowledge than a mentor who directly states lesson feedback. This knowledge would help in the development of a protocol for beginning MLS mentors. No study reviewed investigated the discourse that took place during the debriefing sessions with the MLS mentor present and not present. Interested schools or universities would benefit from this knowledge as the creation of a protocol for MLS mentors may help to maximize the time available during debriefing sessions. Additionally, analyzing the discourse during the debriefing sessions may be useful to understanding whether the MLS mentor is more valuable during early debriefing sessions or in the final session.

\section{Problem Statement}

As lesson study and variations such as MLS are implemented in the United States, it is important to understand key elements of the process to prevent ineffective implementation that could potentially lead educators to discard the technique completely. One element of the process that had not been investigated prior to the present study was the role the outside specialist or MLS mentor plays in relation to growth in participants' mathematical teacher knowledge upon completion of the process. Although the work of M. Fernandez and Zilliox (2011) accentuates the importance of the mentor in providing formative feedback during the lesson study cycles, to date no study reviewed had examined the relation between the type of mentor interaction structure and the outcome of MLS in terms of preservice teacher development of mathematics teacher knowledge. The research supporting the potential of MLS as a process for improving mathematics teacher knowledge, particularly for secondary school teachers, was clear (M. Fernandez, 2005, 2006, 2010), but little was known about the role the MLS mentor plays in facilitating the process. This knowledge gap warranted further study so that mathematics teacher 
educators interested in implementing the process may be equipped with the appropriate knowledge to guide their students through a MLS experience that leads to growth in preservice teacher mathematics teacher knowledge.

\section{Purpose of the Study}

The purpose of the present study was to examine the relation between mentor interaction structure used in MLS and elementary preservice teachers' development of mathematics teacher knowledge. Additionally, this study examined the discourse that takes place during the debriefing sessions when the interaction structure is varied. During previous semesters, elementary preservice teachers have engaged in debriefing sessions with the MLS mentor after both the first and second lesson presentations. In a semester course, this requires allocating a substantial amount of time to the debriefing sessions that could potentially be used for other experiences. Two other interaction structures that require less time and may be equally effective in developing preservice teachers' knowledge for teaching mathematics are possible and were investigated in this study.

A second alternate mentor interaction structure was developed to reduce the number of debriefing sessions involving the mentor to one session following the teaching of the first version of the MLS lesson. This type of interaction structure continues to call on the mentor to provide formative feedback and possibly at the most critical time in the process- following the first lesson teach, but decreases the amount of time required of the MLS mentor. This first debriefing session could serve as a model for the subsequent debriefing sessions to be completed by the MLS groups independently. This type of interaction structure allows group members to autonomously negotiate and refine the MLS lesson, which could potentially strengthen the current process as it allows the participants the opportunity to resolve issues independently. 
A third alternate mentor interaction structure involved the MLS mentor in only the final debriefing session. This type of mentor interaction is more summative in nature and gives individual groups the opportunity to self-direct prior to receiving support from the MLS mentor for analyzing and revising the lesson. Similar to the second interaction structure presented, this alternative interaction structure requires less time from the mentor and could potentially deepen participants' ability to analyze and refine lessons independently as they work to revise their lessons. However, this type of debriefing structure includes the mentor at the end of the process to clarify any unresolved issues that remain after the group has had the opportunity to refine their lesson independently. It is possible that these remaining issues may be more specific and less noticeable to group members and therefore require the guidance of the MLS mentor.

\section{Research Questions}

The following research question guided this study: Is there a difference between three mentor interaction structures used in Microteaching Lesson Study as it relates to the development of elementary preservice teachers' mathematics teacher knowledge?

Two secondary questions investigated in this study were as follows: (1) Is there a relationship between elementary preservice teachers' gains in mathematical content knowledge after participation in one of three mentor interaction structures used in Microteaching Lesson Study and the gains of a comparison group in which the MLS process was not used? (2) What aspects of the discourse in debriefing sessions support the growth of elementary preservice teachers' mathematics teacher knowledge during MLS?

\section{Theoretical Framework}

In order to examine the development of elementary preservice teachers' mathematics teacher knowledge through MLS, gains in content knowledge and pedagogical content 
knowledge in mathematics were investigated. Shulman's (1986) ideas related to subject matter content knowledge were used to analyze growth in mathematics content knowledge. Graeber's (1999) framework for pedagogical content knowledge related to mathematics was used to analyze preservice teacher growth in pedagogical content knowledge for mathematics. This section begins with a description of Shulman's (1986) ideas related to content knowledge and pedagogical content knowledge. Although Shulman's (1986) framework for pedagogical content knowledge was not directly used in the analysis of elementary preservice teacher growth in pedagogical content knowledge for mathematics, a discussion of his ideas is presented as Graeber's (1999) ideas are rooted in his more broad framework. The section concludes with a discussion as to how the work of Shulman (1986) and Graeber (1999) guided the analysis of elementary preservice teacher content and pedagogical content knowledge development.

Subject matter content knowledge, according to Shulman (1986), requires that teachers not only possess an understanding of the facts and concepts related to their respective disciplines, but also be able to explain why a particular topic or concept is worth knowing and how it relates to other topics or concepts not only within their respective discipline, but within other disciplines as well. For mathematics, this would mean possessing both procedural knowledge and conceptual knowledge. Procedural knowledge is an understanding of how to carry out a specific algorithm or calculation. This is distinct from conceptual knowledge in which the student understands why an algorithm works and can explain the meaning behind the procedures.

Pedagogical content knowledge as explained by Shulman (1986) includes two main points. First, teachers should have knowledge of the most useful forms of representation, examples, analogies, illustrations or explanations for the most regularly taught topics in a subject area. Second, Shulman (1986) advocates teachers understand what makes the learning of 
specific topics easy or difficult. This includes knowledge of preconceptions, which may be misconceptions, that students of different ages and backgrounds bring with them into the classroom. Teachers should also have knowledge of strategies that will be helpful in reorganizing student thinking with regard to such preconceptions.

Graeber's (1999) idea of pedagogical content knowledge is encapsulated in terms of five "big ideas", which describe the attributes of a teacher who possesses pedagogical content knowledge in mathematics. While Graeber's (1999) ideas can be linked to Shulman's (1986) framework for pedagogical content knowledge, her perspective is unique in that it offers ideas for fostering the development of each big idea with preservice teachers. The framework outlines five aspects of mathematics pedagogical content knowledge that teacher education programs might consider as they design courses for preservice teachers. Graeber's (1999) five big ideas include the following: (a) "Understanding students' current understanding” (p.192), (b) "Students knowing in one way do not necessarily know in the other(s)" (p.195), (c) "Intuitive knowledge is both an asset and a liability" (p.198), (d) "Certain instructional characteristics appear to promote retention" (p.200), (e) "Alternative representations and the recognition and analysis of alternative methods are important" (p.202).

The first three big ideas are connected to one portion of Shulman's construct for pedagogical content knowledge in which he advocates teachers should have knowledge of the preconceptions or misconceptions related to the most commonly taught topics in their subject area. The last two big ideas are related to the other portion of pedagogical content knowledge; knowing how to make the subject understandable to others.

“Understanding students' current understanding” (p.192), Graeber’s first big idea (1999), means a teacher should possess knowledge of research related to stages involved in the 
development of particular mathematics topics like van Hiele's (van Hiele, 1959) levels of geometric thinking or identified stages in the path to understanding place value. Through knowledge of the stages associated with particular topics, teachers can assess their students' understanding within such hierarchies and create lessons that will help to move their students forward. She also suggests teachers should learn to assess understanding during instruction. Graeber advises that preservice teachers often are not convinced of the need to assess student understanding during a lesson until given the opportunity to work closely with a student who does not understand a particular topic. She suggests preservice teachers be given the opportunity to engage in experiences in which they work with students during the methods course.

Graeber's (1999) second big idea, "students knowing in one way do not necessarily know in the other(s)" (p.195), refers to the idea that students may possess procedural knowledge related to a specific topic, but not necessarily conceptual knowledge. She advocates mathematics teachers engage students in tasks that evoke both procedural and conceptual understanding. Graeber (1999) recommends providing opportunities for preservice teachers to engage students in dialogue aimed at eliciting responses that demonstrate student understanding of both procedural and conceptual knowledge.

The third big idea, "intuitive knowledge is both an asset and a liability" (Graeber, 1999, p.198), is related to Shulman's (1986) assertion that students often bring to the classroom their own preconceptions, which may be misconceptions. For mathematics, Graeber (1999) explains tasks that are designed to develop conceptual knowledge, often evoke intuitive knowledge. For example, a student may intuitively believe multiplication always results in a greater number. Mathematics teachers need to be cognizant of such intuitions as they design their lessons and foster a classroom environment in which students feel comfortable to engage in dialogue that 
may challenge their ideas. Preservice teachers often incorrectly believe student intuitions can be corrected by telling the student the correct idea. To help preservice teachers understand telling students the correct way of thinking will not suffice, Graeber (1999) suggests engaging preservice teachers in actual teaching or tutoring experiences. She also recommends designing tasks or posing problems that will lead preservice teachers to consider the misconception for themselves.

“Certain instructional characteristics appear to promote retention” is Graeber's (1999, p.200) fourth big idea. For this idea, Graeber (1999) suggests mathematics teachers have knowledge as to which instructional strategies will best lead to student retention. Mathematics teachers should recognize that tasks in which students can reflect on their own thinking through writing or speaking are more likely to be meaningful and remembered by students then a teacherled discussion. Graeber (1999) recommends preservice teachers be given time to experience activities that model this approach to instruction as learners and to design lessons that incorporate such strategies.

The last big idea described by Graeber (1999) is "alternative representations and the recognition and analysis of alternative methods are important” (p.202). Within this big idea, Graeber (1999) asserts mathematics teachers need to understand different models or representations will be useful in constructing ideas for different students. Consequently, mathematics teachers need to have knowledge of multiple representations and also design tasks that include different models for the same concept. Graeber (1999) recommends preservice teachers be required to develop lesson plans that include different models for the same concept.

For the purposes of this study, growth in content knowledge was measured using an instrument, see Appendix A, that included questions that capture both conceptual and procedural 
knowledge growth based on Shulman's (1986) ideas related to subject matter content knowledge. To investigate growth in pedagogical content knowledge, lesson plans developed by preservice teachers during the MLS cycle were analyzed according to Graeber's (1999) framework. Findings from the discourse analysis with respect to content knowledge and pedagogical content knowledge were used to triangulate these results.

\section{Significance of the Study}

This study provides knowledge as to which of three possible MLS mentor interaction structures described earlier led to more elementary preservice teacher growth in content knowledge and pedagogical content knowledge in mathematics. This knowledge helped to inform the amount of time required of the MLS mentor when implementing the process. It was possible that the interaction structure used in previous semesters of the mathematics methods course, in which the MLS mentor participated in each debriefing session, was not necessary to preserve sufficient growth in elementary preservice teacher mathematics knowledge for teaching. University instructors and school districts may also benefit from this investigation as it provided knowledge that is useful when making decisions about implementing the process in their respective institutions.

Through analysis of discourse between the MLS mentor and elementary preservice teacher groups during debriefing sessions, it was possible to investigate ways the MLS mentor was a factor in participant learning and outcomes of the process. The discourse analysis was an important piece to investigate as it was possible that aspects of the discourse between the MLS mentor and the MLS groups may have been more effective than others. Knowledge of the existence of effective discourse was helpful in identifying whether a need for developing a training protocol for individuals prior to serving as a MLS mentor was needed. It was also 
important to examine the results of the discourse analysis in order to determine whether further research into what training a MLS mentor would require to be effective in the role is necessary.

\section{Definitions of Key Terms}

Interaction structure: The structure of the participation (or no participation) of an MLS mentor across the three debriefing sessions with individual MLS groups.

Mathematical content knowledge: Possessing an understanding of both the conceptual and procedural knowledge related to a topic.

Mathematical teacher knowledge: For the purposes of this study, will include mathematical content knowledge and pedagogical content knowledge for mathematics.

MLS group: A group of at most three participants who are ideally mixed in ability and pedagogical disposition that engage in cycles of planning, teaching, and revision of a research lesson.

MLS mentor: The person, usually a course instructor or individual considered an expert in their field, who facilitates Microteaching Lesson Study and provides guidance for debriefing sessions, as needed.

Research lesson: A lesson designed to answer a research question aimed at achieving an overarching goal for student learning.

\section{Assumptions and Delimitations of the Study}

This study's assumptions included the following: (a) every preservice teacher can develop their mathematics teacher knowledge; (b) the major topics covered in each of the courses in which MLS was implemented was equivalent, but lessons were delivered in different ways. 
The first delimitation of the study involves the choice to investigate the MLS mentor interaction structure to examine the role of the MLS mentor in producing an experience in which elementary preservice teachers' mathematics teacher knowledge is developed. Other types of involvement of the MLS mentor could have been examined. For example, the MLS mentor could potentially watch each lesson as it is enacted, strictly through video or combinations of both methods rather than the current method of watching pieces of each lesson as it is taught. However, the amount of time invested in the MLS process by the MLS mentor may be significantly reduced through a change in the current interaction structure involved in the implementation. A second benefit is a small reduction in class time allocated for completion of the MLS process. For example, if the MLS mentor is only required to debrief after the first lesson or after the final lesson as described in the alternate interaction structures, then all groups could debrief simultaneously rather than rely on the MLS mentor to participate. While this does not drastically reduce the class time required of the MLS process, it does decrease the class time associated with debriefing.

A second delimination of the study involves the choice of framework to analyze mathematics teacher knowledge based on Shulman's (1986) subject matter content knowledge and Graeber's (1999) version of pedagogical content knowledge for mathematics. While other frameworks for content knowledge that were more specific to mathematics than that of Shulman were examined, it was determined that the current work available divided content knowledge more specifically than needed for this study. Other versions of pedagogical content knowledge were examined as well, but Graeber's (1999) ideas were found to be the best fit as they were based on work with preservice teachers in a methods course as in the case of this study. 
A third delimitation of the study involves the choice not to vary the topics across the interaction structures. While this alternative possibility would have eliminated the possibility that the findings from each interaction structure were a consequence of the difficulty level of the topic, the decision was made to hold the topics constant in order to help control for the effect the different MLS mentors might have on the development of the lessons.

\section{Organization of the Study}

This chapter included the background to the problem, problem statement, purpose statement, and theoretical framework. Also discussed were the significance of the study, definitions of key terms, and assumptions and delimitations of the study. Chapter 2 presents a review of the literature that supported this study. Chapter 3 describes the methods that were used to investigate the research questions. Chapter 4 presents both the quantitative and qualitative results of the study. In Chapter 5, a discussion of the findings of the study, limitations of the research, and recommendations for further research are presented. 


\section{Chapter II}

\section{Literature Review}

This chapter begins with a discussion of the process associated with lesson study as it is typically implemented in Japan followed by a similar discussion describing the process associated with Microteaching Lesson Study (MLS). Next, a review of the studies in which MLS is implemented is presented. As MLS is a relatively new variation of lesson study, it was necessary to review the literature related to lesson study to better understand the development and possible benefits associated with MLS. A review of literature related to lesson study practices with preservice teachers followed by research studies involving lesson study with practicing teachers is discussed. The chapter concludes with a summary of the literature reviewed.

\section{Japanese Lesson Study}

One of the primary sources of information on Japanese lesson study is Stigler and Hiebert's (1999) The Teaching Gap. In the book, a Japanese approach to lesson study involving eight steps is described. An important aspect of Japanese lesson study is the idea that the process is a way for teachers to develop and test hypotheses related to their chosen student learning goal. The hypotheses are predictions about how student weaknesses can be improved through the lesson (C. Fernandez et al., 2003). Consequently, Japanese teachers refer to the lesson as the "research lesson."

The first step in the lesson study process is to define the problem. The problem is usually based on an overarching goal such as fostering students' problem solving skills and responsibility for learning. The source of the problem often originates from a teacher's own experience with students, but can also be designed to address educational policymaker's 
concerns. In one study by C. Fernandez et al. (2003), one of the Japanese advisors who acted as an outside expert for a group of American teachers involved in lesson study, explained that the overarching goal for a lesson study is often developed from teacher conversations about the type of student they want to develop followed by a discussion of student weaknesses in the area of interest. The teachers then talk about ways they or the school can help to improve their student's weaknesses in terms of the overarching goal. The goal may also develop from the lesson study group's examination of teacher's guides from different textbook companies (Takahashi \& Yoshida, 2004). The group will then work to refine the goal so that it can be addressed within a research lesson. The goal becomes the focus of the group meetings and the evaluation of the resulting lesson.

In the second step, the group plans the research lesson. During the planning stage, the group considers potential student solutions as it develops a first version of the lesson. In the third step, one of the group members teaches the lesson while the other members act as observers who record notes with an emphasis on making observations related to student learning. The fourth step begins the reflection portion of lesson study in which the group discusses ways to improve student learning based on their observations. The lesson is then refined in step five and another group member teaches the revised lesson with the non-teaching members again acting as observers in step six. In step seven the group members gather again to evaluate the refined lesson. Steps five, six, and seven may be repeated several times until the group is satisfied with the research lesson. In step eight, a final report is submitted for publication to be shared with the broader community. 


\section{Microteaching Lesson Study}

Microteaching Lesson Study (MLS) is a variation of lesson study that incorporates aspects of microteaching with central elements of lesson study to form an experience that is designed to challenge preservice teachers' conceptions regarding teaching and learning while simultaneously encouraging their connection between theory and practice (M. Fernandez, 2006). Similar to lesson study, the emphasis of MLS is on developing a lesson that is focused on an overarching student learning goal. However, the lesson study goal is chosen purposely by the course instructor to enhance student mathematical teacher knowledge. When the MLS groups teach their peers rather than K-12 students, specific content topics are chosen based on the course instructor's understanding of the preservice teachers' prerequisite knowledge and instructor developed pre-assessment. Topics used in the pre-assessment are selected with two purposes in mind. First, to ensure the experience of teaching their peers is authentic and second, to enhance content knowledge (M. Fernandez, 2005, 2010).

Preservice teachers are assigned a group topic based on the results of a pre-assessment administered prior to engaging in the process. The pre-assessment contains questions designed to gauge preservice teacher content knowledge and pedagogical content knowledge. Heterogeneous groups of at most three are created so that group members are mixed in terms of content knowledge strength and pedagogical disposition (M. Fernandez, 2010). Ideally, a group of three would contain a member with a high, middle, and low ability for the topic as well as a mix of traditional disposition and constructivist disposition toward teaching.

MLS groups are instructed to develop a research lesson based on their assigned topic that may be restricted in length to approximately 30 minutes. The research lesson is subjected to traditional lesson study recursive cycles of plan, implement, analyze and refine. The instructor 
acts as the outside expert or MLS mentor providing feedback and support as needed. As with lesson study, the preservice teachers develop a final product of the process in the form of a written reflective report. Different from typical lesson study, the lesson is videotaped to facilitate the needs of the instructor who may need to observe more than one lesson at the same time, to provide the teacher of the lesson in any given cycle an opportunity to view and better reflect on their own lesson, and to provide a video record of the lesson for the MLS group members to use as needed in analyzing the lesson (M. Fernandez, 2006). Additionally, the lesson is taught in a reduced class size of approximately five to ten students and may be taught to peers (M. Fernandez, 2010).

\section{Research Studies Involving Microteaching Lesson Study}

In an initial investigation into MLS, M. Fernandez (2005) introduced and studied the implementation of MLS with 36 total prospective secondary mathematics teachers enrolled in a mathematics methods course in one of two semesters. In this study, heterogeneous groups of three preservice teachers engaged in a three cycle lesson revision process of MLS. MLS groups were assigned a topic based on their performance on an initial questionnaire. Topics included in the questionnaire were purposefully selected by the MLS mentor to capture secondary preservice teachers' content knowledge weaknesses and pedagogical disposition. During each cycle of MLS, the research lesson was taught by a different MLS group member to a distinct small group of their peers while being videotaped. Following the teaching of the first two lessons, each MLS group engaged in a debriefing session with the MLS mentor. After the final lesson, the MLS mentor provided written feedback to each MLS group. Each MLS group was required to submit a six section final report upon completion of the experience. 
Data sources included MLS group projects, videotapes of lessons, lesson topics questionnaire, a feedback survey, and notes from informal observations. Videotapes of the lessons and written lesson plans, submitted as part of the final report, were coded with respect to pedagogy used and preservice teachers' knowledge of subject matter. To analyze pedagogy, lesson activities were coded based on level of student engagement in developing concepts without preservice teacher 'telling' the relationships. The knowledge of subject matter demonstrated in lesson plan activities for MLS group members was compared with results from the initial questionnaire. The MLS final report and informal observations were coded with respect to preservice teacher growth in content and pedagogy and used to triangulate findings from videotaped lessons and lesson plans. Finally, the feedback survey was used to analyze preservice teachers' perspectives of the MLS experience.

For more than $80 \%$ of the initial MLS lessons, preservice teachers tended to "tell" their student's the relationships related to their topic without providing tasks or activities for their students to develop the ideas themselves. In contrast, after receiving feedback from the MLS mentor during debriefing sessions, less than $20 \%$ of the final lessons involved preservice teachers "telling" their students ideas. Instead, final lessons involved activities in which students could develop or construct relationships through experimentation, exploration of patterns, and use of mathematical reasoning (M. Fernandez, 2005). M. Fernandez (2005) reported the debriefing sessions with the MLS mentor were a key element in this shift. The MLS mentor provided feedback and challenged aspects of the MLS groups' lessons that either group members did not consider or were unwilling to discuss, which pushed the preservice teachers to move toward a more student-centered lesson design. M. Fernandez also noted preservice teachers were often unwilling to substantively critique their peers' lessons. This would indicate the MLS 
mentor may be essential to the process. This study was conducted with secondary preservice teachers; thus, it is unknown whether similar results would be found with elementary preservice teachers. The present study intends to investigate whether the MLS Mentor is an essential element in debriefing sessions with elementary preservice teachers. It will also clarify whether the MLS Mentor is needed at both the first and second debriefing sessions or whether participation in the first session would suffice for fostering the preservice teachers' development.

M. Fernandez and Robinson (2006) captured the perceptions of 74 preservice teachers enrolled in four different sections of an initial course on learning to teach secondary mathematics after participating in MLS. The MLS implementation followed the structure used in the M. Fernandez (2005) study in which preservice teachers were assigned a topic and group based on their performance on an initial questionnaire. Each MLS group then engaged in a three cycle revision of their lesson requiring each member to teach at least one version of the lesson to their peers. Following the teaching of the first or second lesson, the MLS mentor engaged each MLS group in a debriefing session. Written feedback was given to the groups after some of the lessons. A final report was submitted by each MLS group.

Upon completion of the MLS cycles, preservice teachers completed a feedback survey that included both Likert-type items and open-ended questions. For the Likert-type items, preservice teachers were asked to provide an explanation for their ratings. For each Likert-type question, values for the mean and standard deviation were calculated. Explanations for each Likert-type item were analyzed qualitatively to understand the reasons supporting the corresponding mean value. Themes that arose from the explanations of the Likert-type questions were triangulated with findings from the qualitative analysis of the open-ended questions. 
M. Fernandez and Robinson (2006) reported preservice teachers felt the collaboration, analyses, and reflection on shared experiences were beneficial to their learning to teach. Findings also indicated preservice teachers valued the MLS process as an opportunity to put into practice ideas they were learning in their coursework (M. Fernandez \& Robinson, 2006). Mean values for statements supporting these ideas as well as questions related to preservice teachers' perception of their growth in terms of content knowledge from participation in the process were reported. For example, M. Fernandez and Robinson (2006) reported a mean value of 1.65 out of a possible 5 ( 1 indicates strongly agree) for the reverse response item, "Participation in others' lessons did not help me learn math," indicating preservice teachers felt participation in the process helped deepen their understanding of mathematics.

While this study provided support for why MLS is beneficial for preservice teachers, no findings were reported describing preservice teachers' perceptions of the role of MLS mentor in their MLS experience. The feedback survey did not appear to include questions intended to capture preservice teachers' perceptions of the MLS mentor or the specific value of debriefing sessions in contributing to their growth in content or pedagogical content knowledge. This study also investigated the use of MLS with secondary preservice teachers and not elementary preservice teachers as the present study intends to do.

More recently M. Fernandez (2010) investigated the use of MLS with 18 prospective teachers enrolled in a course introducing them to the teaching of mathematics at the secondary school level. For the 2010 study by M. Fernandez, MLS was implemented in a manner congruent with the 2005 and 2006 studies described earlier. Only two differences to the MLS implementation were noted. First, a five section final report was required instead of the previous 
six sections. Second, preservice teachers created individual pre-MLS and post-MLS lesson plans on a designated topic.

Similar to the positive findings associated with lesson study and previous studies with MLS, M. Fernandez (2010) reported preservice teachers' engagement in MLS deepened their understanding of the content and enhanced their ability to teach. According to M. Fernandez, "MLS sufficiently maintained important and authentic aspects of the complexity of typical classroom practice in order to help focus the prospective teachers' attention on content pedagogy and the related student learning while engaging in managing classroom processes" (M. Fernandez, 2010, p.9). Additional benefits reported by M. Fernandez (2010) included the development of preservice teachers' ability to recognize teaching a lesson as a learning process not building a skill, preservice teachers began to recognize textbooks are not the authority on curriculum, and preservice teachers began to integrate classroom management strategies into their lesson design. However, the benefits associated with MLS in this case are pertinent to secondary preservice teachers. The present study seeks to determine whether MLS with elementary preservice teachers will lead to similar results.

In another study by Matthews, Hlas, and Finken (2009), researchers engaged preservice teachers in MLS according to M. Fernandez (2005) with the addition of a four-column lesson plan template as a crucial part of the lesson study. Findings are based on student journal entries made while enrolled in their practicum and methods specific courses as well as comments from the preservice teachers' student teaching supervisor. Student surveys were analyzed at the end of the course and six months later to gain insight into preservice teachers' perceptions of the fourcolumn lesson plan's usefulness in developing lessons. The four-column lesson plan template was reported to be an integral part of the participants' success as it provided a means for 
focusing preservice teachers on student learning as they engaged in the lesson study process. Both M. Fernandez (2005) and Matthews, Hlas, and Finken's (2009) work with preservice teachers engaged in MLS inform how to successfully implement lesson study with preservice teachers. However, these studies were all qualitative in nature and involved prospective secondary mathematics teachers. The present study will involve prospective elementary teachers enrolled in a mathematics methods course and will consist of both quantitative and qualitative approaches to investigate the gains in mathematical teacher knowledge when the MLS mentor interaction structure is varied.

\section{Lesson Study with Preservice Teachers}

At the university level, instructors of methods courses have adapted lesson study for use with preservice teachers. These studies have involved students enrolled in general methods courses as well as subject specific methods courses. Various aspects of lesson study have been examined including its effectiveness for improving teacher pedagogy, content knowledge, efficacy and collaborative ability.

Sims and Walsh (2008) examined the effectiveness of two adaptations of lesson study developed within a two-year study involving preservice teachers enrolled in Foundations of Early Childhood Education. Data acquired during the implementation of the first year adaptation of lesson study was used to inform the implementation structure of the second year version. The first year involved 32 preservice teachers ( 30 undergraduate juniors and 2 graduate students) and the second year involved 25 preservice teachers (24 undergraduate juniors and 1 graduate student). In both cases, one of the researchers acted as the course instructor.

For the initial version of lesson study, the class was divided into three groups by practicum grade level (kindergarten, first, and second/third groups) and groups were instructed to 
choose a lead teacher to develop a research lesson on Venn Diagrams. The lead teacher videotaped their lesson while completing their field experience hours for later group analysis. The other group members adapted and taught the research lesson to meet the needs of their own field experience students.

The researchers reported the initial implementation of lesson study was problematic and made several changes to the implementation of the lesson study during year two to address these issues. First, preservice teachers conducted their lessons with their peers in their university classroom during the first six weeks of the semester. This was done to allow the preservice teachers to view the lesson as it was enacted rather than rely on a videotaped version of the lesson. Second, in contrast to the first implementation, in which the researchers intentionally limited their role, the researchers took a more active role in working with the groups. For example, groups were assigned based on the researcher's understanding of individual strengths and personalities. A third change was made so that the lesson would be based on an assigned chapter of the text associated with the course. Lastly, researchers developed and required the use of an Observation and Evidence Worksheet to help the preservice teachers as they planned and evaluated their lessons.

Three goals were developed to determine the effectiveness of the lesson study variations. These goals included teaching preservice teachers to do the following: 1) Analyze lessons in light of lesson goals; 2) Engage in detailed discussions about instructional strategies (such as questioning techniques, anticipating student responses, and how the lesson flow affects student understanding; 3) Critique the lesson plan, not the teacher. A fourth goal was added during the second year of the study to investigate preservice teachers' ability to observe and gather actual 
evidence about learning. Written notes and video/audiotapes of classroom discussion, planning sessions, and lesson debriefing sessions were analyzed to evaluate the goals of the study.

Findings from this study indicate that the structure of year two was more effective in helping preservice teachers to meet these goals. Researchers reported preservice teachers were able to analyze their lessons in terms of lesson goals and focus on features like questioning and anticipating student thinking when supported during planning and discussion with probing questions from the mentor. This is an important finding as it indicates the importance of the role of the mentor in facilitating an effective lesson study experience for their participants. However, this finding came out of a comparison of the two lesson study versions used in this study. The types of support provided by the mentor that may have contributed to the preservice teachers' development of the skills outlined in the researchers' goals were not investigated. This study will investigate one aspect of mentor support through the investigation of three possible interaction structures during the debriefing sessions and examine the type of discourse used in debriefing sessions that may also be an integral part of the support preservice teachers' need to benefit from their participation in lesson study.

In another study, Parks (2007) engaged 27 preservice teachers enrolled in a 12-week graduate level elementary mathematics methods course in an adaptation of lesson study. The preservice teachers earned their bachelor's degree during the previous spring and were in the process of completing the yearlong teaching internship required by the university in order to earn their teacher certificate. Preservice teachers chose to group themselves according to their elementary field experience school. This resulted in three groups of six and three groups of three. Each group chose from a list of four research goals (adapted from Cochran-Smith 1999) provided by Parks, who acted as the mentor during the lesson study process. 
Parks investigated whether lesson study was an effective tool for the development of preservice teacher thinking about mathematics and equity while designing lesson plans. Three 3hour class periods were devoted to the planning of the research lessons and the mentor provided an agenda with a list of items to consider each day. Groups were given a week and a half to teach their lesson. After teaching the lesson, a class period was devoted to a debriefing session that included either the mentor or field supervisor. Following the debriefing session, each group made a presentation to the class and wrote a final report.

The findings in this study are based on analysis of only four of the six groups, which were selected to represent a range of group sizes, school districts, and grade levels. Although other data sources were available, Parks focused her analysis on the two events with the longest and most focused conversations: the third planning session and the interns' in-class analysis of their lesson after it had been taught. It was not noted whether the mentor or field supervisor participated in the two events.

Both events were audiotaped and transcribed. Codes were developed for content of discussions and participation structure. Each transcript was separated into conversations related to mathematics, pedagogy, students, lesson logistics, lesson study assignment requirements and grading policies, and unrelated topics. This resulted in 181 conversational episodes that were then coded as described earlier. The fewest conversational episodes (21) were spent on mathematics and the majority were about students (38) and pedagogy (39). Sixteen of the 21 mathematics related conversations were from only two groups. In terms of equity, Parks reported only one of the four focal groups engaged in a discussion about teaching to all students while planning their lessons. This group had 18 conversational episodes out of 42 focused on responding to differences among students in ways that accommodate all learners. 
Noting the limited number of conversations related to both mathematics and equity, Parks (2007) suggested that teacher educators may need to think about ways to become more explicit in guiding beginning teachers' work. She also noted that the collaborative nature of lesson study may actually reinforce incorrect ideas or concepts. For example, during one of the planning sessions a group discussion related to estimating and rounding resulted in the reinforcement of incorrect understanding of the differences between the two concepts. Interestingly, this incident happened at a point in the planning session in which Parks had moved to work with another group. This suggests the mentor may need to be present during the entire planning session to ensure preservice teachers develop ideas that are correct. However, no study has investigated whether this is the case. This study will help to fill this gap through an examination of the three interaction structures described earlier.

In another study with preservice teachers, McMahon and Hines (2008) described a lesson study adaptation with eight preservice secondary mathematics teachers as they implemented their lesson with ninth graders in two geometry classrooms. The lesson study adaptation took place within regular school hours on the same day at one large suburban high school. To prepare preservice teachers for the lesson study experience, the authors gave an overview of the steps involved in the process during one of the regular mathematics methods class meetings. Preservice teachers watched a video of a lesson study in progress and were encouraged to think about and ask questions about how lesson study could be used in their clinical setting. Additionally, they read and discussed an article about the implementation of lesson study and its value to improving classroom instruction.

The lesson study topic chosen by the preservice teachers was the Pythagorean Theorem. McMahon and Hines (2008) indicated all eight teachers participated in the same lesson study, 
although they were aware four or five group members is typically recommended in lesson study. The preservice teachers planned their initial lesson over several class sessions. Through their class discussions, they agreed on a lesson sequence that included six parts. During the implementation of the initial lesson, the preservice teacher implementing the lesson modified the order of the intended lesson. This is similar to some of the findings of M. Fernandez (2005); however, upon reflection, lesson revision and re-teaching the preservice teachers still learned from the process.

Although the lesson was observed by the remaining preservice teachers, the regular classroom teacher, the mathematics department head, and the researchers, the debriefing group included only the preservice teachers and researchers. The debriefing session began with the preservice teacher who had implemented the lesson followed by the remaining preservice teachers, and concluded with the researcher's comments. McMahon and Hines (2008) reported the discussion during the debriefing session focused on how well students were grasping concepts. However, the reported changes made to the lesson were related to the order of the lesson activities. Although examples of preservice teacher comments made during the debriefing session were provided, no examples or discussion of the researchers' comments were provided. The second version of the lesson that included the logistical changes discussed in the debriefing session was enacted by a different preservice teacher and took place later the same day during the afternoon geometry class. Only a brief description of this lesson is described in the study, but it was noted that student responses to questions related to the content of the lesson after both lesson versions did not provide evidence that either lesson was more effective in terms of student understanding of the Pythagorean Theorem (McMahon \& Hines, 2008). 
A brief description of the lesson's effectiveness along with one example of the preservice teacher challenging student thinking was described in the study. Further examples or discussion from the researchers would have been helpful to determine whether the first debriefing session was useful in developing preservice teacher's ability to challenge student thinking during the second lesson. This lack of analysis is made apparent by comparison of an attitude survey administered before and after the lesson study process which contained two questions designed to capture preservice teacher ideas related to student thinking. The comparison revealed preservice teachers ideas were unchanged for one question, but notably different in responding to a second question regarding student learning.

During the second lesson debriefing, McMahon and Hines (2008) used several comments made by the preservice teachers to conclude the session had contributed to growth in their understanding of the Pythagorean Theorem. Again comments made by the researchers during the debriefing session were not included, so it is not clear to what extent if at all the researchers contributed to this growth. The present study will help to fill this gap by examining the discourse between the MLS Mentor and preservice teachers.

Post and Varoz (2008), a university assistant professor and district mathematics teacher specialist respectively, engaged preservice teachers enrolled in an elementary mathematics methods course along with practicing teachers enrolled in a district-sponsored professional development course in lesson study. Groups consisted of at least one practicing teacher and between three and six preservice teachers. Both the preservice teachers and practicing teachers met separately in their respective classes for the first 8 weeks of the semester. Then, following initial separation the preservice teachers and practicing teachers worked collaboratively on one cycle of lesson study over a 6-day period. 
Each group consisted of at least one practicing teacher and three to six preservice teachers. The study specifically reported on the first-grade group and specified that the experiences of this group were representative of the other groups. Also, it is noted that the first grade group was the largest group, consisting of three practicing teachers and six preservice teachers, which is large for lesson study. Each group developed their own goals, concepts, and lesson plan. During the planning sessions, Post and Varoz (2008) noted the preservice teachers seemed to allow the practicing teachers to lead the discussions related to choice of activities. However, the preservice teachers participated more actively in developing the actual lesson content such as in choice of questions.

One of the practicing teachers taught the initial group lesson, while the other group members observed. Following the initial lesson, the group discussed the lesson and agreed on revisions. Initially, the discussion seemed to focus on logistics, but in later revisions the group began to focus on student solutions. Researchers reported that the preservice teachers, who initially struggled with anticipating student responses, began to contribute more ideas during the second planning session (Post \& Varoz, 2008).

At the end of the lesson study cycle, participants resumed regular class and engaged in activities that were intended to build on the lesson study experience. Post and Varoz (2008) reported that preservice teachers found the group collaboration was beneficial in seeing a lesson evolve and improve. Findings from this study also indicate that preservice teachers valued the peer collaboration aspect of lesson study and that the practicing teachers benefited from working with the preservice teachers who brought new ideas to the group. However, both the practicing and preservice teachers indicated that the group size made it challenging to collaborate. Findings from this study seem to indicate that attention to group size in lesson study is an important factor 
in facilitating a lesson study that supports equal member participation. The present study will limit MLS groups to at most three members.

While all of these studies aimed to examine the effectiveness of lesson study with preservice teachers, the different researchers reported on the unique ways in which they dealt with issues encountered as they implemented their versions of lesson study. All of these studies will help to inform the methodology of the present study.

\section{Lesson study with Practicing Teachers}

A portion of the research available on lesson study investigates the use of lesson study as it is typically done in Japanese schools with veteran teachers. All studies reviewed employed only qualitative methods. The qualitative nature of the available studies has allowed researchers to explore lesson study from a variety of angles. For example, in one study conducted by $\mathrm{C}$. Fernandez et al. (2003), researchers engaged veteran U.S. and Japanese teachers in an authentic lesson study experience. Their findings centered more around critical lenses the participants developed for examining lesson study effectiveness rather than on the mechanism behind the lesson study experienced by the participating teachers. However, the study does note the importance of outside specialists or mentors in providing support for lesson study participants' implementation of these lenses.

In another study written by the actual teachers who participated in what was intended be an authentic lesson study experience, Taylor, Anderson, Meyer, Wagner and West (2005), reported on the improvements they made to their teaching as a consequence of their participation in lesson study. While both studies provide useful information for those considering implementing lesson study, neither study provides a sufficient model for lesson study use in the U.S. in which the role of the Mentor is explored. 
In the C. Fernandez et al. (2003) study, 16 teachers and administrators from an urban public school in New Jersey worked in conjunction with 12 Japanese teachers from the Greenwich Japanese School in Connecticut. The Japanese teachers acted as the mentors and served as lesson study coaches for the American teachers. The data used to support the findings in this study are based on the fifth and sixth grade teachers who collaborated on a lesson in which students examined ways to calculate the area of triangles. It is not clear from the study how many teachers were part of this group.

Japanese teachers first taught the American teachers basic elements of lesson study practice and then advised and assisted them as they worked on an actual lesson study. C. Fernandez et al. (2003) watched videos of the American and Japanese interactions in real time and created a log of major events. Written artifacts were categorized according to their content and relationship to the video. The research team then reviewed the data and identified instances of Japanese teachers explicitly or implicitly giving American teachers instructions. Advice clusters were created and then coded according to the principles about lesson study practice that the American teachers seem to emphasize. The research team concluded that this data could be grouped into three lenses: the researcher lens, the curriculum developer lens, and the student lens.

Findings suggest that the American teachers did not develop the researcher lens that the Japanese teachers possessed. Researchers based this conclusion on several observations. The American teachers did not develop a hypothesis for their lesson study. They were focused on the logistics of their lesson while Japanese teachers emphasized the importance of the research process. During their planning sessions, American teachers would often engage in debates over how to teach the lesson. The Japanese teachers suggested the classroom could be used to 
experimentally settle their disagreements. However, in all but one group, the American teachers did not use their classroom to test any of their ideas. In contrast to the Japanese teachers who prioritized data collection, American teachers took few notes during the lesson delivery. As a final concern, American teachers were unable to detail what they learned from the lesson study experience.

American teachers also failed to develop the curriculum lens to the extent of the Japanese teachers. Researchers based their findings regarding the curriculum lens on their conclusion that American teachers did not see the importance of the sequence of lessons that the Japanese teachers so greatly valued. The Japanese teachers insisted on visiting the school for an entire day before beginning the planning sessions with the American teachers. They observed lessons and spent time talking to teachers and students. The Japanese teachers explained the purpose of the visit was to learn what was being taught and how it was being taught across all the subjects and grades. Essentially, the Japanese teachers were focused on developing the curriculum of the school whereas the American teachers were concerned primarily with the single lesson they were creating. Additionally, during the planning sessions American teachers did not discuss how the lesson they were developing was connected to the overall mathematics curriculum. In one discussion regarding the sequencing of the lesson, the American teachers explained they had allowed the order of the text to guide their curriculum decisions indicating they did not possess the curriculum lens that the Japanese teachers clearly demonstrated.

The American teachers also did not display characteristics that indicated they had adopted the student lens. An important aspect of lesson study is the focus on student solutions. While the American teachers did consider possible student solutions prior to the lesson implementation, they did not discuss what these solutions conveyed with regard to students' 
understanding and how it was facilitated by the teachers. In contrast the Japanese teachers addressed these issues in the debriefing session that followed the first lesson implementation. The second lesson debriefed provided further evidence that the American teachers had failed to realize the importance of examining student solutions as they had failed to realize the new issues that students had while solving the revised problems. Essentially, the American teachers had not shifted their thinking to view their lesson from a student lens even though the Japanese teachers had tried to emulate it for them in the first planning session.

The American teacher's inability to view their lesson through the three lenses indicates that having teachers engage in lesson study is not enough to improve their teaching. The data in this study strongly suggest that the implementation of lesson study in the U.S. will present substantial challenges for teachers (C. Fernandez et al., 2003). While the evidence in this study suggests that the American teachers did benefit from the interactions with the Japanese teachers, a recommendation that the U.S. develop its own coaches who are familiar with the issues and practices of U.S. teachers is also reported as being important to the future of U.S. lesson study use (C. Fernandez et al., 2003).

In the Taylor et al. (2005) study, the authors described their own experiences as they engaged in a lesson study that was designed to model the traditional Japanese version. The group consisted of four experienced teachers each with 10 to 25 years of teaching experience and one teacher educator, who was a teacher education faculty member at the nearest state university in a rural town in Illinois. The main focus of their paper was to report on six findings that support the goals of what they considered reform mathematics. The teachers involved in the study had been trying to shift their focus from a teaching focus to a learning focus. 
The six findings reported in the Taylor et al. (2005) study include the following: (a) Meeting regularly to plan and teach a research lesson resulted in an effective detailed lesson plan that led to improved student learning; (b) The lesson study model utilized provided a structure that facilitated collaborative planning; (c) The time provided allowed for sharing and reassessment of common practices; (d) Observation of a lesson facilitated the shift from the teaching focus to the learning focus; (e) The focus on student thinking supported the goals of reform mathematics; and (f) Lesson study transformed the participating teachers' working relationship and conversations. Overall, the authors reported the lesson study process empowered and motivated them since they were not being told by mentors about a new method and were able to shape their own professional development to meet their needs.

Despite their reported success, the authors cited four areas of concern. First, the more the teachers collaborated during the lesson study, the more they became frustrated with external mandates that they believed acted counter to the best interest of students and may have been the reason they had not focused on student learning previously. Next, they noted the process of shifting from traditional practice to reform was challenging. Third, in their view, understanding the goals of lesson study took time and experience and lastly, administrative support is a necessary component for successful implementation of lesson study.

While the findings reported by Taylor et al. (2005) provide evidence that lesson study can be effective in the U.S. as well as point out areas of concern that should be addressed, the role of the teacher educator is not sufficiently examined. This is similar to the C. Fernandez et al. (2003) study that also does not examine the role of the mentor. Both studies do report on the respective mentors' involvement, but neither study explores how their participation influenced the reported results of each study. 


\section{Chapter Summary}

MLS research, research involving other variations of lesson study with preservice teachers, and research with practicing teachers over the last decade indicate the effectiveness of United States lesson study variations. Researchers report positive findings indicating that the benefits associated with the professional development technique are substantial. These benefits include improved content knowledge for teachers, enhancement of teacher pedagogical content knowledge, higher teacher self-efficacy and motivation, development of teacher ability to observe and focus on student learning, improved teacher reflection ability, and creation of collaborative networks for teachers (C. Fernandez, Cannon, \& Chokshi, 2003; Lewis et al., 2004; M. Fernandez, 2005, 2006, 2010; Taylor et al., 2005; Lewis et al., 2006; Puchner \& Taylor, 2006; Parks, 2007).

Current lesson study research has focused on examining the outcome of the technique without a thorough examination of the mechanism that led to the results (C. Fernandez et al., 2003; Lewis et al., 2006). One aspect of the mechanisms involved in the lesson study process that has not been sufficiently examined is the role the mentor plays in ensuring a positive outcome. More specifically, current research has not sufficiently explored the role of the mentor in achieving a positive lesson study experience for the teachers involved. This study will help to fill this gap by investigating one aspect of the role of the MLS mentor, the interaction with participants during the debriefing sessions, and its relation to gains in mathematical teacher knowledge. 


\section{Chapter III}

\section{Method}

\section{Purpose of the Study}

The purpose of this study was to investigate the relation between MLS mentor interaction structure employed and the development of elementary preservice teachers' mathematics teacher knowledge during Microteaching Lesson Study. More specifically, three possible MLS mentor interaction structures were examined. These interaction structures were as follows: (a) MLS mentor participates in every debriefing session; (b) MLS mentor participates in only the first debriefing session; and (c) MLS mentor participates in only the last debriefing session. A secondary purpose of this study was to investigate aspects of the discourse that fostered preservice teacher development of content and pedagogical content knowledge. Discourse between the MLS mentor and group members during debriefing sessions as well as between group members in the absence of the MLS mentor was examined for differences and similarities.

\section{Research Questions and Hypothesis}

The following research question guided this study: Is there a difference between three mentor interaction structures used in Microteaching Lesson Study as it relates to the development of elementary preservice teachers' mathematics teacher knowledge?

Two secondary questions investigated in this study were as follows: (1) Is there a relationship between elementary preservice teachers' gains in mathematical content knowledge after participation in one of three mentor interaction structures used in Microteaching Lesson Study and the gains of a comparison group in which the MLS process was not used? (2) What aspects of the discourse in debriefing sessions support the growth of elementary preservice teachers' mathematics teacher knowledge during MLS? 
As the first question was partially examined using quantitative methods, the null hypothesis was as follows:

$H_{0}$ : After participation in lessons taught by groups in one of three MLS mentor interaction structures, there is no difference in elementary preservice teachers' gain in mathematical content knowledge.

The first secondary question was also examined using quantitative methods and the null hypothesis was as follows:

$H_{0}$ : After participation in one of three MLS mentor interaction structures or a comparison group in which the MLS process was not used, there is no difference in elementary preservice teachers' gain in mathematical content knowledge.

\section{Research Design}

To investigate the relation between each of the three variations of MLS mentor interaction structures and mathematical teacher knowledge, a sequential mixed-methods research design approach was employed. As described in Hesse-Biber (2010), Greene, Carachelli, and Graham offer five reasons for employing a mixed-methods research design that include triangulation, complementarity, development, initiation, and expansion. Triangulation, which is the use of more than one method to examine a research question, adds to the credibility of research findings when a convergence of all the data from distinct sources is found. Complementarity also serves to support the study's findings as the blend of both qualitative and quantitative data sources provides the researcher an opportunity to capture a more complete picture of their research data. Development refers to the idea that data from one source may inform or guide the next phase in a research study. As an example, statistical data gained from a quantitative aspect of a mixed-methods research approach may be used to generate questions for 
a later interview in a research study. Initiation is the idea that through analysis of multiple data sources in a research study new questions or areas in need of clarification may arise prompting the need for another study. Analysis of multiple data sources may also lead to a need for expansion of an idea uncovered in the investigation. Both initiation and expansion help inform future studies.

In terms of this study, a mixed methods research design was best suited to understand the relationship between mentor involvement and elementary preservice mathematical teacher knowledge for the triangulation, complementarity and development reasons. Triangulation was accomplished through use of the feedback survey and discourse analysis to support findings from the content and pedagogical content knowledge pieces of the study. The use of both quantitative and qualitative analysis at various stages in the study achieves complementarity. As the results of the quantitative analysis of mathematical content knowledge growth were used to inform the choice of MLS groups selected for the qualitative analysis, development was accomplished.

\section{Participants}

The participants in this research study were 103 elementary preservice teachers, including 72 participating in MLS and 31 in a comparison group not participating in MLS. The 72 participants, 1 man and 71 women, participating in MLS were enrolled in three different sections of a mathematics methods course entitled Content and Methods of Teaching Elementary Mathematics for Grades 1 to 6 (MAE 4310) at an urban, Hispanic-serving university in the south during the Spring 2011 semester. Twenty-eight participants, all female, were enrolled in the first section of the course, which was taught by the researcher and met on Monday, Wednesday, and Friday from 10:00 to 10:50 a.m. Twenty-one participants, one male and twenty female, were enrolled in the second section of this course, which was taught by a male doctoral candidate and 
met on Monday, Wednesday, and Friday from 11:00 to 11:50 a.m. Twenty-three participants, all female, were enrolled in the third section of the course, which was taught by a female doctoral candidate and met on Tuesday and Thursday from 2:00 to 3:15 p.m.

The thirty-one elementary preservice teachers, all female, not participating in MLS were enrolled in two different sections of the same mathematics methods course taught by two adjunct instructors. As part of their course requirements, members of these sections did not participate in MLS and as such were used as a comparison group for a portion of this study. Both sections met once a week in the evening for 2 hours and 40 minutes during the same semester as the participants who completed MLS in their courses.

At the time of the study, the course instructors for those sections in which MLS was a requirement were all in the later stage of completing a Ph.D. in Curriculum and Instruction with a focus in mathematics education at the same university. Each of the instructors had taught the course prior to engaging in this study and had served as the MLS mentor in prior semesters. Each of the course instructors acted as the MLS mentor for the groups in their respective sections.

As one of the female course instructors, I have taught various mathematics and mathematics methods courses ranging from grade 5 to university level for eleven years. I have a Bachelor's degree in Mathematics and a Master's of Science in Mathematics Education. The other female doctoral student has 11 years teaching experience in elementary, middle, and university level courses. She has a Bachelor's degree in Mathematics and English Education and a Master's of Education in Educational Leadership. The male doctoral student has taught mathematics education courses at the university level at times during his 4 years enrolled in the doctoral program. He has a Bachelor's degree in Secondary Mathematics Education and 
completed his student teaching at the high school level. He is enrolled in a program that will yield a doctoral degree without a formal Master's degree.

\section{MLS Implementation}

During the first week of the semester prior to the MLS experience, elementary preservice teachers completed an initial pre-assessment, see Appendix A, which contained questions designed to assess elementary preservice teachers' content knowledge and pedagogical content knowledge. For the initial part of the semester, elementary preservice teachers watched videos, read case studies, and engaged in class discussions and activities all modeling a reform-oriented approach to mathematics teaching and at the same time intended to deepen their understanding of mathematics content taught in elementary school.

The initial pre-assessment contained 10 total questions related to the 9 topics that were available for assignment in each section. Of the 10 questions, two questions were related to the same topic and the other eight were each related to a single topic, see Appendix A. The instrument had been used in previous semesters and had undergone several revisions so that each question(s) pertaining to a mathematical idea would gauge participants' understanding of both the conceptual and procedural knowledge when possible for that topic. However, it should be noted that for the topics corresponding to questions 7 and 10, only procedural understanding was measured as the question used in the instrument did not sufficiently capture conceptual understanding. To establish validity of the instrument, two other mathematics educators reviewed the questions for content knowledge as defined in this study. Both mathematics educators agreed the instrument appropriately measured mathematical content knowledge for each of the 9 chosen topics. 
Based on elementary preservice teacher responses to the initial pre-assessment and the MLS mentor's understanding of each participant's pedagogical disposition or orientation toward teacher-directed or constructivist approach instruction through class interactions and assignments, groups were created of at most three so that members were mixed in ability and pedagogical approach. The goal was to group elementary preservice teachers so that each MLS group would contain members whose understanding of the topic were varied and whose pedagogical approach ranged between teacher-directed and constructivist instruction. In this manner, a total of 26 groups were formed. This included ten groups from Section 1, seven groups from Section 2, and nine groups from Section 3.

For each course section, the MLS mentor assigned a topic along with an overarching student learning goal to each MLS group. Topics chosen were based on the researcher and instructors' experience with gaps in elementary preservice teachers' knowledge of mathematics during previous semesters, as well as information from the initial pre-assessment. Within each course, the topics assigned were the same. Examples of topics included multiplying fractions without the use of an algorithm or developing the connection between the area formulas for parallelogram, triangle, and trapezoid. In connection to their assigned topic, each MLS group, for all three sections, were also assigned one of the two following overarching goals: 1) To develop students' ability to build new mathematical knowledge through problem solving by applying or adapting a variety of appropriate strategies, or 2) To develop students' mathematical reasoning and ability to study patterns in constructing relationships or concepts through experimenting, analyzing, conjecturing, and defending or justifying mathematical ideas.

It should be noted that, elementary preservice teachers engaged in MLS at the end of the semester they enrolled in MAE 4310, after completing the course curriculum. Topics assigned to 
MLS groups in each interaction structure were chosen so that each interaction structure had a topic connected to ideas presented early in the semester, during the semester, and later in the semester prior to engaging in MLS. While assigned topics were not directly covered during the course, they were connected to ideas that had been presented.

At the start of the MLS experience, time was allocated in class for MLS groups to develop the first versions of their research lesson for their assigned topics and goals. During this time, the respective MLS mentor consulted with each group and posed questions to help guide participant thinking as they created their research lesson. Each MLS group developed, taught to their peers, and revised three versions of their research lesson on their assigned topic. Each group member taught one of the lessons, while the other two members acted as either observer or video recorder-observer. Group members decided the order in which they taught in the three teaching cycles. All lessons were video recorded and given to the respective groups for viewing and analysis.

Two groups taught their lessons simultaneously during class sessions. For example, in Table 1, teachers from Group 1 and Group 2 taught their lessons simultaneously. The students for Teacher 1a consisted of all members in Groups 3, 5, 7, and 9 and the students for Teacher 2a consisted of all members in Groups 4, 6, 8, and 10. Table 1 is an example of a schedule for a course that meets three times a week for 50 minutes. A similar schedule was used for Section 1 and Section 2 of this study. However, for Section 2, the schedule was shortened to reflect seven groups. The schedule was adjusted for Section 3 as the class met only two times a week for 75 minutes each.

For Table 1, eight class days are devoted to the teaching of the research lessons. Each day, four groups present their lessons. Two groups deliver their lessons simultaneously and the 
MLS mentor views pieces of each lesson as they are enacted. If the corresponding course intstructor deemed it necessary, a video-taped version of the lesson was available for viewing. While no data was collected with respect to how often the MLS mentors in this study viewed videos in this manner, it did occur. This enabled the MLS mentor to provide observation notes or engage in dialogue during debriefing sessions as described in the respective interaction structure detailed in the Purpose section of Chapter 1.

Table 1

Example Schedule for MLS Implementation

\begin{tabular}{|l|l|l|l|l|l|l|l|l|l|l|}
\hline & Teacher & Students & Teacher & Students & & & Teacher & Students & Teacher & Students \\
\hline Day 1 & $1 \mathrm{a}^{*}$ & $3,5,7,9$ & $2 \mathrm{a}$ & $4,6,8,10$ & Day 5 & $8 \mathrm{~b}$ & $1,3,5,9$ & $7 \mathrm{~b}$ & $2,4,6,10$ \\
\hline & $3 \mathrm{a}$ & $1,5,7,9$ & $4 \mathrm{a}$ & $2,6,8,10$ & & $10 \mathrm{~b}$ & $1,3,5,7$ & $9 \mathrm{~b}$ & $2,4,6,8$ \\
\hline Day 2 & $5 \mathrm{a}$ & $1,3,7,9$ & $6 \mathrm{a}$ & $2,4,8,10$ & Day 6 & $1 \mathrm{c}$ & $2, \ldots{ }^{* *}$ & $3 \mathrm{c}$ & $4, \ldots$ \\
\hline & $7 \mathrm{a}$ & $1,3,5,9$ & $8 \mathrm{a}$ & $2,4,6,10$ & & $2 \mathrm{c}$ & $1, \ldots$ & $4 \mathrm{c}$ & $3, \ldots$ \\
\hline Day 3 & $9 \mathrm{a}$ & $1,3,5,7$ & $10 \mathrm{a}$ & $2,4,6,8$ & & Day 7 & $5 \mathrm{c}$ & $6, \ldots$ & $8 \mathrm{c}$ & $7, \ldots$ \\
\hline & $2 \mathrm{~b}$ & $3,5,7,9$ & $1 \mathrm{~b}$ & $4,6,8,10$ & & $6 \mathrm{c}$ & $5, \ldots$ & $9 \mathrm{c}$ & $10, \ldots$ \\
\hline Day 4 & $4 \mathrm{~b}$ & $1,5,7,9$ & $3 \mathrm{~b}$ & $2,6,8,10$ & Day 8 & $7 \mathrm{c}$ & $8, \ldots$ & $10 \mathrm{c}$ & $9, \ldots$ \\
\hline & $6 \mathrm{~b}$ & $1,3,7,9$ & $5 \mathrm{~b}$ & $2,4,8,10$ & & & & & \\
\hline
\end{tabular}

"The number indicates the group and the letter indicates the version of the lesson. So, 1a indicates group 1, lesson version 1.

${ }^{* *}$ Other groups may be assigned to participate in lesson as designated by the MLS mentor.

Following the teaching of each lesson, within each course, each MLS group engaged in a debriefing session with their mentor and MLS group members, see Table 2, according to one of the three interaction structures described earlier. Based on the enrollment in each course, a total of eight groups were assigned to the first interaction structure, including three groups from the first section, two groups from the second section, and three groups from the third section. Similarly, eight groups were assigned to the second interaction structure, including three groups 
from the first section, two groups from the second section, and three groups from the last MLS section. Lastly, ten groups were assigned to the third interaction structure, including four groups from the first section, three groups from the second section, and three groups from the third section.

Table 2

Groups per Interaction Structure

\begin{tabular}{|c|c|c|c|c|}
\hline $\begin{array}{c}\text { Interaction } \\
\text { Structure }\end{array}$ & Section 1 & Section 2 & Section 3 & Number of Groups \\
\hline 1 & 3 & 2 & 3 & 8 \\
\hline 2 & 3 & 2 & 3 & 8 \\
\hline 3 & 4 & 3 & 3 & 10 \\
\hline Total & 10 & 7 & 9 & 26 \\
\hline
\end{tabular}

In the case of the first described interaction structure, each MLS mentor was asked to begin the debriefing session with the MLS group observer, who shared recorded observations related to the effectiveness of the lesson. It should be noted that each group member was required to serve as the group observer during one of the lessons. The choice to have the group observer begin the session was made for two reasons. First, the group observer was required to record their observations and so may present more details than perhaps the other members and second, it sets the tone that the debriefing sessions were to be driven by data. The MLS mentor was instructed to use the observer's ideas to generate a discussion amongst group members so as to address any issues or concerns related to the lesson. In the case that group members were unable to resolve an issue related to content or pedagogical content knowledge, the MLS mentor was instructed to pose questions to help guide the MLS group to a resolution. For the second interaction structure, the MLS mentor was asked to follow a similar method for the debriefing session, but only participated in the first debriefing session. Lastly, the MLS mentor participated in only the final debriefing session with groups assigned to the third interaction structure. 
Participants were required to submit a final report, see Appendix B, based on the structure of the final report used with secondary preservice teachers (M. Fernandez, 2005, 2010) and included the original three versions of the research lesson plan developed by the group along with a group reflection for each debriefing session. Also, included in the final report was a discussion of initial issues the group experienced while developing their first lesson and an analysis of each lesson implementation. Lastly, participants completed the MLS feedback survey, see Appendix C, also based on the structure of the feedback survey used with secondary preservice teachers (M. Fernandez, 2005, 2010) to capture their perceptions of how the MLS process helped develop their content and pedagogical content knowledge based on their assigned interaction structure.

\section{Data Collection and Analysis}

As discussed previously, mathematical teacher knowledge was investigated in terms of content knowledge as described by Shulman (1986) and pedagogical content knowledge as described by Graeber (1999). The analysis began with the investigation of elementary preservice teachers' growth in mathematical content knowledge. First, participants from courses in which MLS was not a requirement completed the same initial pre-assessment that MLS mentors administered in their respective sections and used to guide the MLS group assignments. The instrument, as discussed earlier, contained questions related to the MLS groups' assigned topics, topics that were part of the curriculum of the course, MAE 4310. All participants completed the initial pre-assessment again as a post-assessment. This administration of the post-assessment corresponded to the end of the MLS cycle for those course sections implementing the MLS process. It should be noted that all participants completed the same initial pre and postassessment instrument, although participants within two of the sections where MLS was required 
did not participate in lessons related to all the topics as only one section of the course had sufficient participants to create group lessons for all 10 topics. Also, all sections of the course completed the post-assessment, which contained 11 questions to accommodate the need to add another topic in one of the course sections as a student who had anticipated dropping the class due to medical reasons made the decision to remain in the course. This decision was made in order to ensure each MLS group did not have more than three group members.

As discussed earlier, mathematical content knowledge was investigated based on conceptual and procedural understanding of selected mathematics topics. For consistency in scoring, a rubric was created detailing how to score the pre and post assessment for both aspects of understanding, see Appendix D. Scores from the procedural and conceptual portion of each topic were summed for a total score. It should be noted, for two of the topics, the respective questions used in the pre and post assessment instrument only captured procedural understanding. Also, only participants who completed both the pre and post assessment were used for analysis. Using the rubric, a second MLS mentor involved in the study, independently scored a random sample of 10 total pre and post-assessments and an inter-rater reliability coefficient was calculated.

To determine if elementary preservice teachers' mathematical content knowledge had developed independent of participation in MLS, two separate paired t-tests were completed. First, a paired t-test based on total scores from the initial pre-assessment and the corresponding ten questions on the post-assessment was done for participants enrolled in the courses in which MLS was not a part of their course requirements. Similarly, a second paired t-test based on total scores from the initial pre-assessment and post-assessment was done for participants who did complete MLS as part of their course. A Type 1 error of $\alpha=.05$ was set, so that statistical 
significance will be found at $p<.05$. Descriptive statistics were also calculated for both MLS groups and non-MLS groups.

Next, to understand if there was a difference in mathematical content knowledge between participants in those sections who did complete MLS in their course from those who did not, an Analysis of Covariance (ANCOVA) was done. The choice to use ANCOVA for analysis was made to help control for the compounded Type 1 error rate associated with performing multiple paired t-tests and to increase the power of the test (Hinkle, Wiersma, \& Jurs, 2003). Scores on the initial pre-assessment for all 103 participants were used as the covariate, participation in MLS was the independent variable, and performance on the post-assessment was the dependent variable. A Type 1 error of $\alpha=.05$ was set, so that statistical significance will be found at $p<$ .05 .

Then, to better understand and measure elementary preservice teachers' mathematical content knowledge growth as a result of their participation in the MLS experience, the initial preassessment, which was also used as a post-assessment, was administered to participants enrolled in courses only where MLS was required prior to beginning the MLS process. This was done the class session before participants had begun to teach their MLS lessons. This second preassessment contained eleven questions and was administered as the post-assessment for both the comparison group and MLS group. It should be noted that although the same instrument was used three times during the semester, the time between the administration of each instrument was approximately 12 weeks and 3 weeks respectively.

Gain scores from the second pre-assessment and post-assessment for the MLS participants were calculated for the topics corresponding to each interaction structure. Both descriptive and inferential statistics were used to analyze the results of this second pre- 
assessment and post-assessment. Again, to help control for the compounded Type 1 error rate associated with performing multiple paired t-tests and to increase the power of the test, an Analysis of Variance (ANOVA) was used to analyze the gain scores (Hinkle et al., 2003). A Type 1 error of $\alpha=.05$ was set, so that statistical significance will be found at $p<.05$.

To conclude the analysis of mathematical content knowledge, each MLS group member's percent of change for their corresponding lesson topic from the second pre-assessment to the post-assessment was calculated and then an average group percent of change was found. Then, for each interaction structure, each MLS group was ranked from least average group percent of change to greatest average percent of change for their respective topic. This knowledge was later used to help select groups for analysis of discourse during debriefing sessions.

Next, to determine growth in pedagogical content knowledge, each MLS group's lesson plans and group reflections after each debriefing session (included in the MLS final report) were analyzed using the Graeber (1999) framework. A rubric was created, see Appendix E, in which each of Graeber's big ideas were further defined so as to allow for a rater to assign a score of low, medium, or high to the lesson plan for each big idea. This rubric was used to code the lesson plans and group reflections. Then, an overall code for each of the five big ideas was determined for each lesson plan by noting which code appeared most often in the particular lesson plan and group reflection.

Of the total 26 groups who participated in the study, one group had two members who did not complete the course. This resulted in a total of 25 groups that completed the three lesson cycle and were available for analysis, eight groups from the first interaction structure, seven groups from the second interaction structure, and 10 groups from the third interaction structure. Data from each group were compiled and frequency tables were created illustrating for each big 
idea the total number of low, medium, and high lessons for each interaction structure. Bar graphs of the data were made to provide a visual representation of the frequency tables. Both the frequency tables and bar graphs were used to develop themes for discussion.

Then, to support the findings from the content and pedagogical content knowledge analysis, results from the analysis of a feedback survey, see Appendix C, completed by elementary preservice teachers upon completion of the MLS process were used. The feedback survey was intended to capture elementary preservice teachers' perspectives of how participation in MLS helped develop both their content and pedagogical content knowledge as well as how their MLS mentor interaction structure contributed to their mathematical teacher knowledge.

Included in the feedback survey were a series of Likert-type format questions requiring a numerical rating ranging from 1 to 5 along with an explanation of the rating chosen. A score of 1 corresponded to a rating of Strongly Disagree, a score of 2 corresponded to a rating of Disagree, a score of 3 corresponded to a rating of Neutral, a score of 4 corresponded to a rating of Agree, and a score of 5 corresponded to a rating of Strongly Agree. Items were phrased in both the negative and positive version of the statement. Descriptive statistics were used to analyze the Likert-type question portion of the survey and supporting responses were reviewed to ensure responses corresponded to the chosen numerical value. As all responses coincided with the participants' numerical rating, no adjustments were made to their scores.

Lastly, the transcripts of the debriefing sessions of 12 MLS groups were selected for analysis based on the results from the content analysis. Using total MLS group gains from the pre-assessment administered prior to MLS and the post-assessment for each MLS groups' performance for the questions related to their topic, four groups from each of the three interaction structures were selected. As some participants did not complete either the pre- 
assessment or the post-assessment, only 23 groups were available for selection. For each interaction structure, transcripts from the two MLS groups with the highest overall gain and the two MLS groups with the least overall gain from the pre-assessment to the post-assessment were chosen for coding. It should be noted that in the case when two or more MLS groups had the same total gain, the instructor was used as a factor in deciding which group would be analyzed to ensure all instructors would be represented in the data.

Coding was completed according to the process described by Miles and Huberman (1994) in which first-level coding is descriptive followed by development of second-level or pattern codes and concluding with the derivation of more general themes through a process known as memoing. An a-priori list of codes for first-level coding was developed, but later expanded as needed during the data analysis, see Appendix F, in which the MLS Mentor actions were classified separate from the elementary preservice teachers' actions. The second-level coding involved reviewing the descriptive codes for patterns. Themes were developed for discussion in the third-level of coding. Bar graphs were made to visually represent the patterns.

\section{Limitations}

As this study was based on a convenience sample, results must be interpreted carefully. Also, it could be argued that the individual qualifications of the MLS mentor may play a role in the outcome of the MLS experience. Although, the three MLS mentors in this study were all pursuing a doctoral degree in the same field, no information is provided as to their pedagogical dispositions. It is possible that a mentor with a more traditional or constructivist disposition may interact with elementary preservice teachers in different ways, thereby affecting the development of mathematical teacher knowledge. 
Additionally, no information was provided with regard to MLS mentor content knowledge. A MLS mentor with a stronger background in mathematics may be able to facilitate deeper mathematical learning with their corresponding groups then a mentor with less mathematical knowledge, who might miss opportunities to enhance elementary preservice teacher mathematical knowledge.

Another limitation of the study was the use of the same instrument three times during the semester. Participants' scores may increase because of their experience with previous administrations of the instrument (Gall, Gall, \& Borg, 2007). Also, the instrument only required preservice teachers to complete one question per topic, which may be problematic as a careless error on their parts may erroneously skew the analysis of their results. 


\section{Chapter IV}

\section{Results}

This chapter describes the findings of a parallel mixed-methods study intended to investigate the relation between MLS mentor interaction structure and elementary preservice teacher growth in mathematical teacher knowledge. Data sources included an initial preassessment, a second pre-assessment, and a post-assessment, a MLS final report including group developed lesson plans, transcripts of audio-recorded debriefing sessions, and a feedback survey. The chapter begins with the results of the quantitative analysis of the initial pre-assessment, the second pre-assessment, and the post-assessment followed by findings from the qualitative analysis of the lesson plans, MLS final report, and discourse during the debriefing sessions. The chapter concludes with findings from the analysis of the feedback survey, which included both a quantitative and qualitative piece.

\section{Mathematical Content Knowledge Analysis}

To begin the analysis of elementary preservice teacher mathematical content knowledge growth, a power analysis was done to determine the chance that given a small, medium, and large effect size occurred in the population, a sample size of 72 participants and $\alpha=.05$ would detect it. Given these values, power for a small effect size $\left(f^{2}=.02\right)$ was found to be less than 0.2. For a medium effect size $\left(f^{2}=.15\right)$, power was found to be approximately 0.89 , and for a large effect size $\left(f^{2}=.35\right)$, power was found to be greater than 0.99 . This means given $\mathrm{n}=72$ and $\alpha=.05$, if a medium effect is present in the population, there is approximately an $89 \%$ chance it will be found. 
Then, the initial pre-assessment, the second pre-assessment, and the post assessment were scored by me. Also, a second MLS mentor involved in the research process scored five randomly selected second pre-assessments and five randomly selected post-assessments. An inter-rater reliability coefficient was found to be $r=.973$ and significant for $r<.01$. Both evaluators used a rubric to score the instrument, see Appendix D. The initial pre-assessment contained ten total questions, while the second pre-assessment and post-assessment contained eleven total questions. This included the original ten questions used in the initial pre-assessment and one extra question added, as discussed earlier, to accommodate the need for another group in one of the MLS sections.

As more topics were associated with certain interaction structures, a weighted score was calculated by summing the points associated with topics from each interaction structure and then dividing by three. The three resultants for the initial pre-assessment were then added for a final total score of 7. The same method was used to calculate the total possible points for the second pre-assessment and post-assessment and resulted in a final total score of at most $7 \frac{2}{3}$ or approximately 7.67 .

Table 3, below, details the mean and standard deviation values for the initial preassessment and post-assessment for the weighted total score for the MLS and non-MLS groups. For consistency, only the ten questions on the post-assessment that corresponded to the initial pre-assessment were used to calculate the mean values. For the MLS group, the average weighted total score for the pre-assessment was 2.77 with a standard deviation of 1.28 . The average weighted post-assessment score for the total score was 3.62 with a standard deviation of 1.22. For the non-MLS group, the average weighted pre-assessment score for the total score was 1.27 with a standard deviation of 0.74 . The average weighted post-assessment score for the 
average weighted total score for the post-assessment was 1.72 with a standard deviation of 1.05 . While the average total scores from the pre-assessment to the post-assessment indicate both the MLS and Non-MLS groups did grow in the understanding of the mathematical content measured by the instrument, it is important to note that results for the MLS group presented here represent all interaction structures.

Table 3

Average Weighted Scores for Initial Pre and Post Assessment for MLS and non-MLS groups

\begin{tabular}{|c|c|c|c|c|}
\hline & $\begin{array}{l}\text { Average } \\
\text { Weighted Pre- } \\
\text { Assess Total } \\
\text { Score* }\end{array}$ & $\begin{array}{l}\text { Standard } \\
\text { Deviation Pre- } \\
\text { Assess }\end{array}$ & $\begin{array}{l}\text { Average } \\
\text { Weighted Post- } \\
\text { Assess Total } \\
\text { Score* }\end{array}$ & $\begin{array}{l}\text { Standard } \\
\text { Deviation Post- } \\
\text { Assess }\end{array}$ \\
\hline MLS Group & 2.77 & 1.28 & 3.62 & 1.22 \\
\hline $\begin{array}{c}\text { Non-MLS } \\
\text { Group }\end{array}$ & 1.27 & 0.74 & 1.72 & 1.05 \\
\hline
\end{tabular}

*Highest possible score was 7 .

Next, to determine whether the growth in mathematical content knowledge was significant within each of the groups separately, a paired t-test was done comparing scores from the initial pre-assessment and post-assessment for the MLS group and then, the non-MLS group. The results of the paired t-test for the MLS group was significant at $p<.01$. Similarly, the results of the paired t-test for the non-MLS group was also significant at $p<.01$ indicating both groups' growth in mathematical content knowledge over the semester was significant.

Then, to determine if the growth in mathematical content knowledge for both the MLS group and the non-MLS group over the semester was related to participation in MLS, an ANCOVA was completed. The weighted total score on the initial pre-assessment was the covariate, the weighted total score on the post-assessment was the dependent variable, and the group assignment (MLS or non-MLS) was the independent variable. Results of the ANCOVA 
indicated there was a significant difference between the non-MLS group and the MLS group for growth in mathematical content knowledge at $p<.01$.

Then, to understand growth in mathematical content knowledge when the interaction structure was varied, results of the second pre-assessment and post-assessment were analyzed. A score for all topics corresponding to the respective interaction structure was calculated and converted to a percent. Therefore, each participant had three scores, one for each of the respective interaction structures. Table 4, below, details the average values and standard deviations for the second pre-assessment and post-assessment as percent values per interaction structure. The average second pre-assessment scores expressed as percentages for each interaction structure were (1) 36.81, (2) 51.51, and (3) 38.33 with standard deviations of 19.17, 25.50, and 21.10, respectively. The average post-assessment scores expressed as percentages for each interaction structure were (1) 46.53, (2) 62.27, and (3) 52.78 with standard deviations of $19.07,23.11$, and 22.55, respectively.

Table 4 Average Weighted Scores as Percents for Second-Pre and Post Assessment

\begin{tabular}{|c|l|l|l|c|}
\hline $\begin{array}{c}\text { Interaction } \\
\text { Structure }\end{array}$ & $\begin{array}{l}\text { Average } \\
\text { Weighted Pre- } \\
\text { Assess Total } \\
\text { Score }\end{array}$ & $\begin{array}{l}\text { Standard } \\
\text { Deviation Pre- } \\
\text { Assess Total } \\
\text { Score }\end{array}$ & $\begin{array}{l}\text { Average } \\
\text { Weighted } \\
\text { Post-Assess } \\
\text { Total Score* }\end{array}$ & $\begin{array}{l}\text { Standard } \\
\text { Deviation } \\
\text { Post-Assess } \\
\text { Total Score }\end{array}$ \\
\hline 1 & 36.81 & 19.17 & 46.53 & 19.07 \\
\hline 2 & 51.51 & 25.50 & 62.27 & 23.11 \\
\hline 3 & 38.33 & 21.10 & 52.78 & 22.55 \\
\hline
\end{tabular}

An ANOVA was used to analyze the elementary preservice teacher performance gains from the questions related to each assigned interaction structure on the second pre-assessment to the post-assessment. However, significance was not found $(p=0.175)$. Results of the ANOVA 
suggest that elementary preservice teachers' understanding of the mathematics topics assessed by the second pre and post assessment developed regardless of the interaction structure assigned. However, it is important to note that topics were not varied across interaction structures. Therefore, it is possible that certain topics may have been more challenging than others, which would result in less overall gain for that interaction structure.

\section{Pedagogical Content Knowledge Analysis}

To analyze growth in pedagogical content knowledge, lesson plans and group reflections included in each MLS group's final report were coded according to the rubric, see Appendix E, created based on Graeber's five big ideas. Items appearing in the lesson plans and group reflections related to each big idea were classified as either low, medium, or high as described in the rubric. As discussed earlier, lesson plans for 25 of the 26 groups were coded for analysis as one group had two members who did not complete the course and no final report was submitted. This resulted in eight groups from the first interaction structure, seven groups from the second interaction structure that were coded, and 10 groups from the third interaction structure that were coded for analysis.

\section{Big Idea \#1: Understanding students' current understanding}

For Graeber's first big idea, understanding students' current understanding, the percent of lessons coded as low, medium, and high for each lesson in each interaction structure was

calculated, see Table 5. A graphical representation of these results can also be found in Figure 1. 
Table 5

Percentage of Low, Medium, and High Lessons per Interaction Structure for Big Idea \#1:

Understanding students' current understanding

\begin{tabular}{|l|r|r|r|r|r|r|r|r|r|}
\hline & \multicolumn{3}{|c|}{ Interaction Structure \#1 } & \multicolumn{3}{c|}{ Interaction Structure \#2 } & \multicolumn{3}{c|}{ Interaction Structure \#3 } \\
\hline & $\begin{array}{l}\text { Lesson } \\
\text { Plan } \\
\text { \#1 }\end{array}$ & $\begin{array}{l}\text { Lesson } \\
\text { Plan \#2 }\end{array}$ & $\begin{array}{l}\text { Lesson } \\
\text { Plan \#3 }\end{array}$ & $\begin{array}{l}\text { Lesson } \\
\text { Plan \#1 }\end{array}$ & $\begin{array}{l}\text { Lesson } \\
\text { Plan \#2 }\end{array}$ & $\begin{array}{l}\text { Lesson } \\
\text { Plan \#3 }\end{array}$ & $\begin{array}{l}\text { Lesson } \\
\text { Plan \#1 }\end{array}$ & $\begin{array}{l}\text { Lesson } \\
\text { Plan \#2 }\end{array}$ & $\begin{array}{l}\text { Lesson } \\
\text { Plan \#3 }\end{array}$ \\
\hline Low & 12.5 & 12.5 & 12.50 & 28.57 & 28.57 & 28.57 & 30 & 40 & 30.00 \\
\hline Med & 87.5 & 62.5 & 25.00 & 57.14 & 28.57 & 28.57 & 30 & 20 & 30.00 \\
\hline High & 0 & 25 & 62.50 & 14.29 & 42.86 & 42.86 & 40 & 40 & 40.00 \\
\hline
\end{tabular}

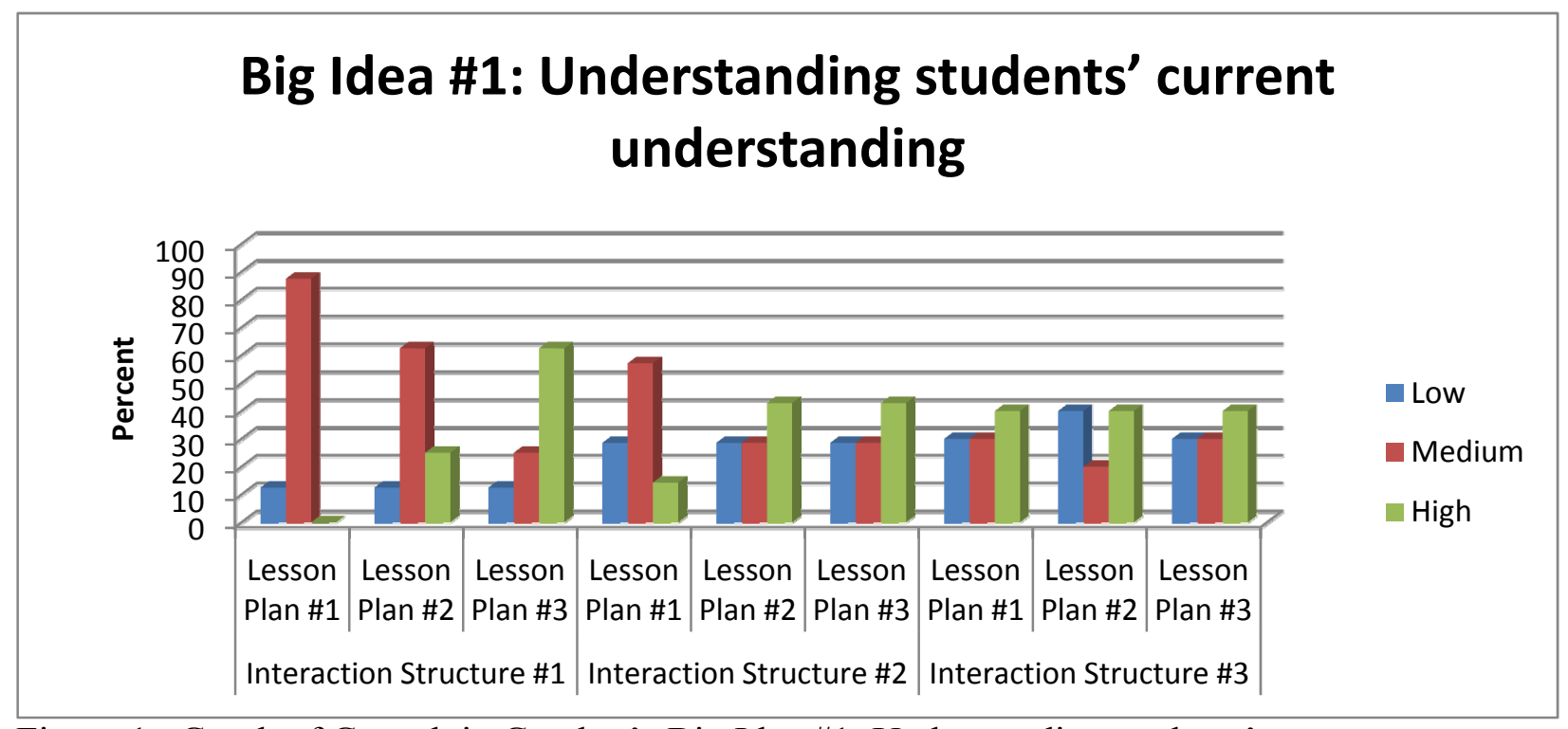

Figure 1. Graph of Growth in Graeber's Big Idea \#1: Understanding students' current understanding per interaction structure.

For the first interaction structure, the percent of low, medium, and high first lesson plans were $12.5 \%, 87.5 \%$, and $0 \%$, respectively. The percent of low, medium, and high for the second lesson plans were $12.5 \%, 62.5 \%$, and $25 \%$, respectively and the percent of low, medium, and high for third lesson plans were $12.5 \%, 25 \%$, and $62.5 \%$, respectively. Comparison of the percent of high lesson plans in the third lesson plan to the first lesson plan indicates participants grew substantially in their ability to plan lessons aimed at understanding students' current understanding from the first lesson. As the first interaction structure, allowed for the first and second debriefing sessions to include the MLS mentor, this growth is important to note. 
For the second interaction structure, the percent of low, medium, and high first lesson plans were $28.57 \%, 57.14 \%$, and $14.29 \%$, respectively. The percent of low, medium, and high second lesson plans were $28.57 \%, 28.57 \%$, and $42.86 \%$, respectively and remained the same for the third lesson plan. As the second interaction structure, allowed for the inclusion of the MLS mentor only after the first debriefing session, it is important to note that no growth occurred between the second and third debriefing sessions. This indicates participants did not grow in their ability to write lesson plans aimed at understanding students' current understanding after the second lesson.

For the third interaction structure, the percent of low, medium, and high first lesson plans were $30 \%, 30 \%$, and $40 \%$, respectively. The percent of low, medium, and high second lesson plans were $40 \%, 20 \%$, and $40 \%$, respectively and the percent of low, medium, and high third lesson plans were $30 \%, 30 \%$, and $40 \%$, respectively. Comparison of the percent of high lesson plans in the third lesson plan to the first lesson plan indicates participants did not grow in their ability to plan lessons aimed at understanding students' current understanding from the first lesson. These findings are important as the third interaction structure did not include the MLS mentor in the debriefing sessions until after all lessons had been taught.

Results of the analysis of growth in pedagogical content knowledge within the different interaction structures revealed the most growth in elementary preservice teachers' ability to plan lessons aimed at understanding students' current understanding in the first interaction structure, the structure that included the MLS mentor in the first and second debriefing sessions.

\section{Big Idea \#2: Students knowing in one way do not necessarily know in the other(s)}

For Graeber's second big idea, students knowing in one way do not necessarily know in the other(s), the percent of lessons coded as low, medium, and high for each lesson in each 
interaction structure was calculated, see Table 6. A graphical representation of these results can also be found in Figure 2.

Table 6

Percentage of Low, Medium, and High Lessons per Interaction Structure for Big Idea \#2:

Students knowing in one way do not necessarily know in the other(s)

\begin{tabular}{|l|r|r|r|r|r|r|r|r|r|}
\hline & \multicolumn{2}{|c|}{ Interaction Structure \#1 } & \multicolumn{2}{c|}{ Interaction Structure \#2 } & \multicolumn{2}{c|}{ Interaction Structure \#3 } \\
\hline & $\begin{array}{l}\text { Lesson } \\
\text { Plan \#1 }\end{array}$ & $\begin{array}{l}\text { Lesson } \\
\text { Plan \#2 }\end{array}$ & $\begin{array}{l}\text { Lesson } \\
\text { Plan \#3 }\end{array}$ & $\begin{array}{l}\text { Lesson } \\
\text { Plan \#1 }\end{array}$ & $\begin{array}{l}\text { Lesson } \\
\text { Plan \#2 }\end{array}$ & $\begin{array}{l}\text { Lesson } \\
\text { Plan \#3 }\end{array}$ & $\begin{array}{l}\text { Lesson } \\
\text { Plan \#1 }\end{array}$ & $\begin{array}{l}\text { Lesson } \\
\text { Plan \#2 }\end{array}$ & $\begin{array}{l}\text { Lesson } \\
\text { Plan \#3 }\end{array}$ \\
\hline Low & 12.5 & 12.5 & 0.00 & 42.86 & 28.57 & 28.57 & 50 & 20 & 30.00 \\
\hline Medium & 87.5 & 62.5 & 62.50 & 42.86 & 71.43 & 42.86 & 30 & 80 & 50.00 \\
\hline High & 0 & 25 & 37.50 & 14.29 & 0.00 & 28.57 & 20 & 0 & 20.00 \\
\hline
\end{tabular}

\section{Big Idea \#2: Students knowing in one way do not necessarily know in the other(s)}

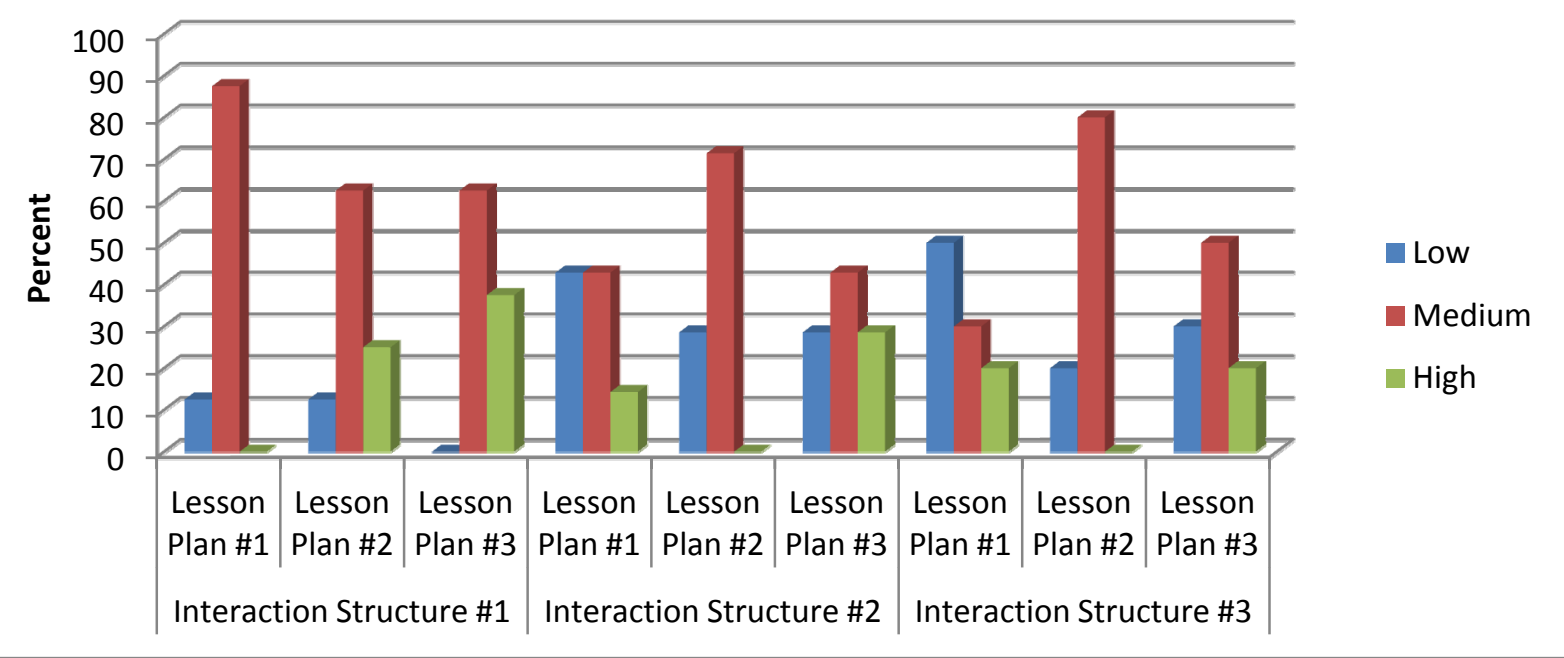

Figure 2. Graph of Growth in Graeber's Big Idea \#2: Students knowing in one way do not necessarily know in the other(s).

For the first interaction structure, the percent of low, medium, and high first lesson plans were $12.5 \%, 87.5 \%$, and $0 \%$, respectively. The percent of low, medium, and high second lesson plans were $12.5 \%, 62.5 \%$, and $25 \%$, respectively and the percent of low, medium, and high third lesson plans were $0 \%, 62.5 \%$, and $37.5 \%$, respectively. Comparison of the percent of high lesson plans in the third lesson plan to the first lesson plan indicates participants grew 
substantially in their ability to plan lessons aimed at developing their students' understanding of not only the procedures relevant to their topic, but also the concept. Additionally, no lesson was considered 'low' by the third lesson plan. As the first interaction structure, allowed for the first and second debriefing sessions to include the MLS mentor, this growth is important to note.

For the second interaction structure, the percent of low, medium, and high first lesson plans were $42.86 \%, 42.86 \%$, and $14.29 \%$, respectively. The percent of low, medium, and high second lesson plans were $28.57 \%, 71.43 \%$, and $0 \%$, respectively and $28.57 \%, 42.86 \%$, and $28.57 \%$, respectively, for the third lesson plan. When compared to the first interaction structure, the number of high lesson plans was less and a substantial amount of lesson plans remained low for the third teach. This means elementary preservice teachers' assigned to the second interaction structure continued to develop lessons aimed at developing primarily procedural understanding of their topic.

For the third interaction structure, the percent of low, medium, and high first lesson plans were $50 \%, 30 \%$, and $20 \%$, respectively. The percent of low, medium, and high second lesson plans were $20 \%, 80 \%$, and $0 \%$, respectively and the percent of low, medium, and high third lesson plans were $30 \%, 50 \%$, and $20 \%$, respectively. Similar to the results presented for the second interaction structure, participants in third interaction structure still developed a substantial amount of lessons aimed at developing only procedural understanding of their topic. Also, when compared to the first interaction structure, the number of third lesson plans that were considered high was less.

Results from the analysis of the lesson plans and group reflections for Graeber's second big idea, students knowing in one way do not necessarily know in the other(s), suggest elementary preservice teachers may need the support of the MLS mentor during the first two 
debriefing sessions in order to maximize their ability to design lessons aimed at developing conceptual understanding in their students. Although, participants assigned to the second and third interaction structure did produce third lesson plans that were considered high, it is important to note that no third lesson in the first interaction structure was considered low. This means elementary preservice teachers in the first interaction structure did not design third lesson plans that were only aimed at developing procedural knowledge in their students.

\section{Big Idea \#3: Intuitive knowledge is both an asset and a liability}

For Graeber's third big idea, intuitive knowledge is both an asset and a liability, the percent of lessons coded as low, medium, and high for each lesson in each interaction structure was calculated, see Table 7. A graphical representation of these results can also be found in

Figure 3.

Table 7

Percentage of Low, Medium, and High Lessons per Interaction Structure for Big Idea \#3: Intuitive knowledge is both an asset and a liability

\begin{tabular}{|l|r|r|r|r|r|r|r|r|r|}
\hline & \multicolumn{3}{|c|}{ Interaction Structure \#1 } & \multicolumn{2}{c|}{ Interaction Structure \#2 } & \multicolumn{3}{c|}{ Interaction Structure \#3 } \\
\hline & $\begin{array}{l}\text { Lesson } \\
\text { Plan \#1 }\end{array}$ & $\begin{array}{l}\text { Lesson } \\
\text { Plan \#2 }\end{array}$ & $\begin{array}{l}\text { Lesson } \\
\text { Plan \#3 }\end{array}$ & $\begin{array}{l}\text { Lesson } \\
\text { Plan \#1 }\end{array}$ & $\begin{array}{l}\text { Lesson } \\
\text { Plan \#2 }\end{array}$ & $\begin{array}{l}\text { Lesson } \\
\text { Plan \#3 }\end{array}$ & $\begin{array}{l}\text { Lesson } \\
\text { Plan \#1 }\end{array}$ & $\begin{array}{l}\text { Lesson } \\
\text { Plan \#2 }\end{array}$ & $\begin{array}{l}\text { Lesson } \\
\text { Plan \#3 }\end{array}$ \\
\hline Low & 50 & 37.5 & 37.50 & 57.14 & 85.71 & 85.71 & 60 & 70 & 60.00 \\
\hline Medium & 50 & 37.5 & 50.00 & 42.86 & 14.29 & 14.29 & 30 & 20 & 20.00 \\
\hline High & 0 & 25 & 12.50 & 0.00 & 0.00 & 0.00 & 10 & 10 & 20.00 \\
\hline
\end{tabular}




\section{Big Idea \#3: Intuitive knowledge is both an asset and a liability}

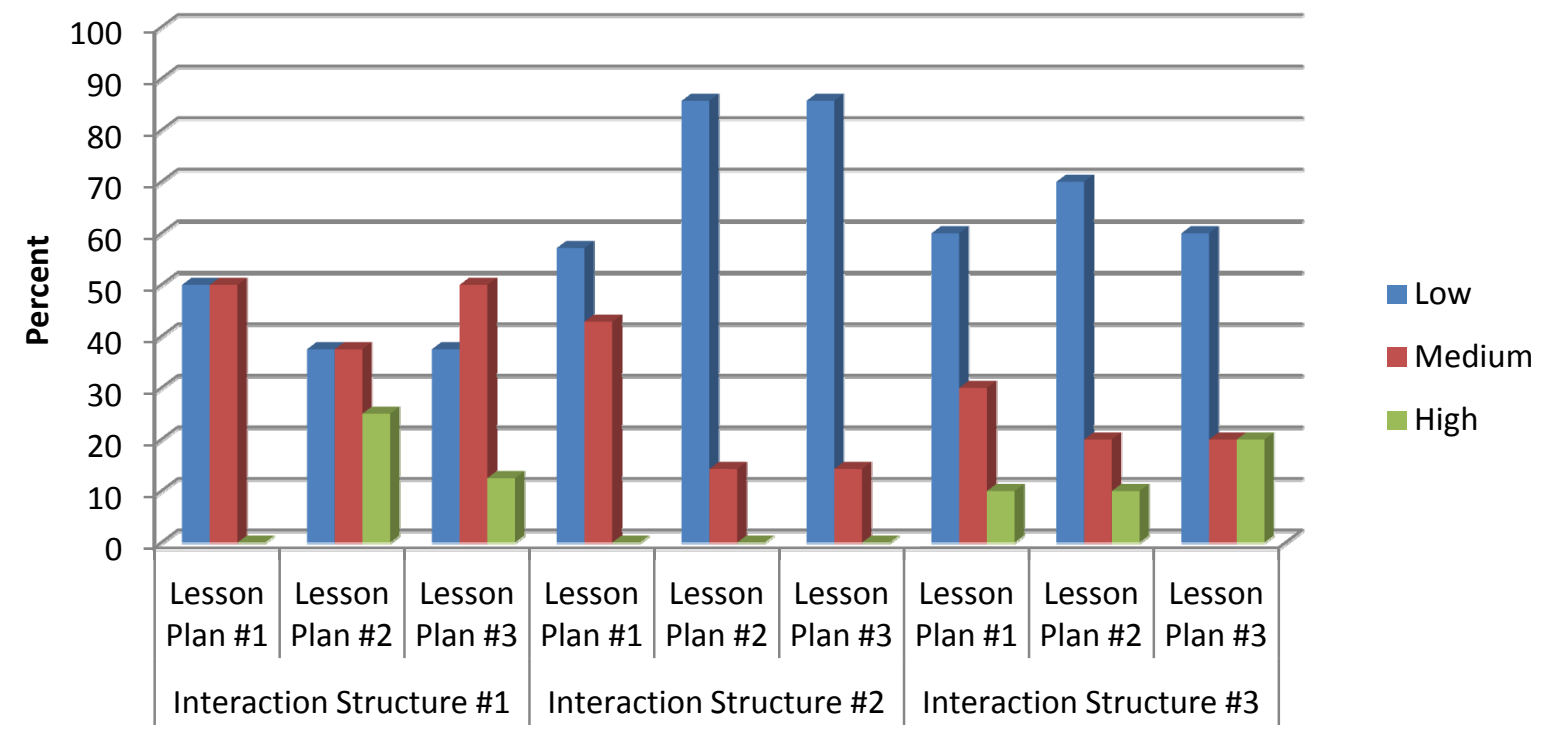

Figure 3. Graph of Growth in Graeber's Big Idea \#3: Intuitive knowledge is both an asset and a liability.

For the first interaction structure, the percentage of first lesson plans classified as low, medium, and high were $50 \%, 50 \%$, and $0 \%$, respectively. The percent of low, medium, and high second lesson plans were $37.5 \%, 37.5 \%$, and $25 \%$, respectively and the percent of low, medium, and high third lesson plans were $37.5 \%, 50 \%$, and $12.5 \%$, respectively. While the results of the analysis of this big idea for the first interaction structure are not as impressive as the findings related to the first two big ideas, it is notable that most third lesson plans were considered medium and high, but had begun as mostly low and medium for the first lesson plan. This would indicate that the MLS mentor was useful in helping most participants assigned to the first interaction structure develop their ability to design lessons in which their students' intuitive knowledge is considered.

For the second interaction structure, the percent of low, medium, and high first lesson plans were $57.14 \%, 42.86 \%$, and $0 \%$, respectively. The percent of low, medium, and high 
second lesson plans were $85.71 \%, 14.29 \%$, and $0 \%$, respectively and $85.71 \%, 14.29 \%$, and $0 \%$, respectively, for the third lesson plan. Results of the analysis of the lesson plans related to this structure are startling, as it would seem in the absence of the MLS mentor not only did participants fail to grow in their ability to plan lessons in which their students' intuitive knowledge is considered, but their ability seemed to regress as the second and third lesson plans contained more low lessons than the first lesson plans.

For the third interaction structure, the percent of low, medium, and high first lesson plans were $60 \%, 30 \%$, and $10 \%$, respectively. The percent of low, medium, and high second lesson plans were $70 \%, 20 \%$, and $10 \%$, respectively and the percent of low, medium, and high third lesson plans were $60 \%, 20 \%$, and $20 \%$, respectively. Similar to the results of the second interaction structure analysis, most participants designed lessons throughout the three teach cycle that did not consider their students' intuitive understanding of the topic. Although, there was growth from the first to the third lesson plans, it was minimal.

Results of the analysis of the lesson plans and group reflections for Graeber's third big idea, intuitive knowledge is both an asset and a liability, suggest that elementary preservice teachers' require substantial support from the MLS mentor in order to develop their ability to design lessons that consider their students' intuitive knowledge. Most participants assigned to interaction structures two and three continued to design lessons throughout the three lesson cycle that did not consider their students' intuitive knowledge at all indicating the need for more support from the MLS mentor.

\section{Big idea \#4: Certain instructional characteristics promote retention}

For Graeber's fourth big idea, certain instructional characteristics promote retention, the percent of lessons coded as low, medium, and high for each lesson in each interaction structure 
was calculated, see Table 8. A graphical representation of these results can also be found in

Figure 4.

Table 8

Percentage of Low, Medium, and High Lessons per Interaction Structure for Big Idea \#4:

Certain instructional characteristics appear to promote retention

\begin{tabular}{|l|r|r|r|r|r|r|r|r|r|}
\hline & \multicolumn{3}{|c|}{ Interaction Structure \#1 } & \multicolumn{2}{c|}{ Interaction Structure \#2 } & \multicolumn{3}{c|}{ Interaction Structure \#3 } \\
\hline & $\begin{array}{l}\text { Lesson } \\
\text { Plan \#1 }\end{array}$ & $\begin{array}{l}\text { Lesson } \\
\text { Plan \#2 }\end{array}$ & $\begin{array}{l}\text { Lesson } \\
\text { Plan \#3 }\end{array}$ & $\begin{array}{l}\text { Lesson } \\
\text { Plan \#1 }\end{array}$ & $\begin{array}{l}\text { Lesson } \\
\text { Plan \#2 }\end{array}$ & $\begin{array}{l}\text { Lesson } \\
\text { Plan \#3 }\end{array}$ & $\begin{array}{l}\text { Lesson } \\
\text { Plan \#1 }\end{array}$ & $\begin{array}{l}\text { Lesson } \\
\text { Plan \#2 }\end{array}$ & $\begin{array}{l}\text { Lesson } \\
\text { Plan \#3 }\end{array}$ \\
\hline Low & 25 & 12.5 & 25.00 & 42.86 & 57.14 & 57.14 & 50 & 50 & 40.00 \\
\hline Medium & 62.5 & 62.5 & 25.00 & 57.14 & 28.57 & 14.29 & 30 & 40 & 40.00 \\
\hline High & 12.5 & 25 & 50.00 & 0.00 & 14.29 & 28.57 & 20 & 10 & 20.00 \\
\hline
\end{tabular}

\section{Big Idea \#4: Certain instructional characteristics appear to promote retention}

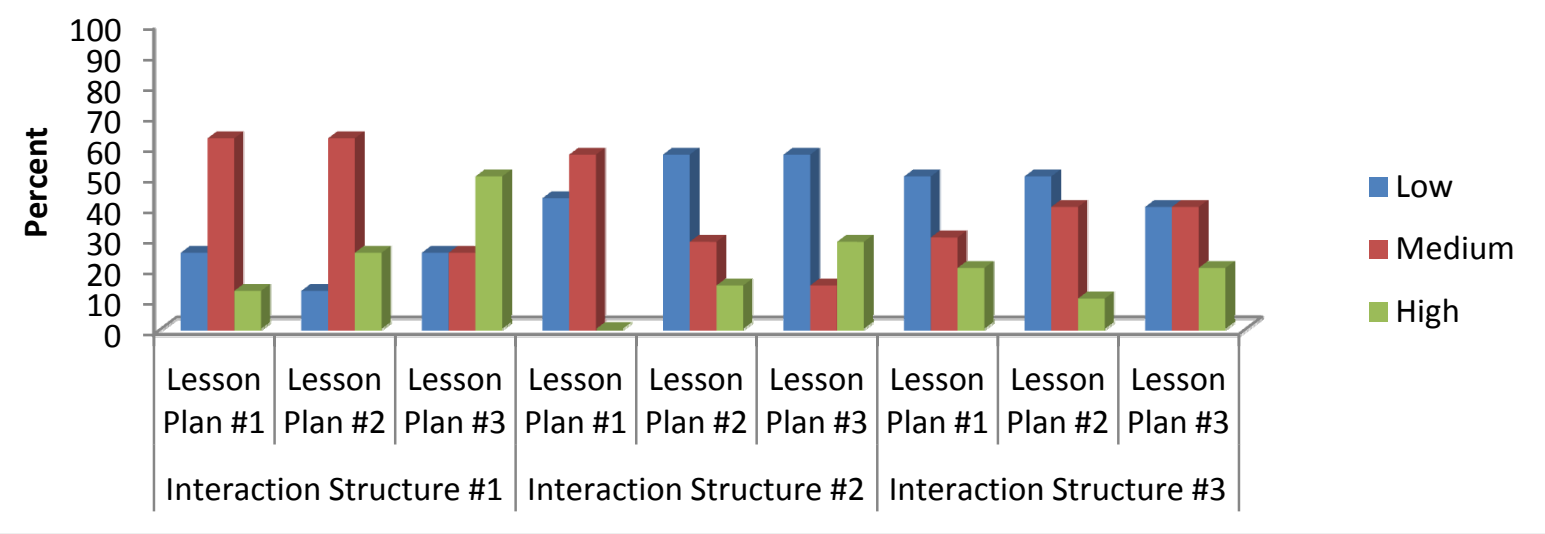

Figure 4. Graph of Growth in Graeber's Big Idea \#4: Certain instructional characteristics appear to promote retention.

The percentage of first lesson plans classified as low, medium, and high for the first interaction structure were $25 \%, 62.5 \%$, and $12.5 \%$, respectively,. The percent of low, medium, and high second lesson plans were $12.5 \%, 62.5 \%$, and $25 \%$, respectively and the percent of low, medium, and high third lesson plans were $25 \%, 25 \%$, and $50 \%$, respectively. Most notable from these results was the increase in percentage of lessons considered high from the first to the third lesson. This would indicate that the MLS mentor was valuable helping participants assigned to 
the first interaction structure develop their ability to design lessons to include instructional strategies that promote retention amongst their students.

For the second interaction structure, the percent of low, medium, and high first lesson plans were $42.86 \%, 57.14 \%$, and $0 \%$, respectively. The percent of low, medium, and high second lesson plans were $57.14 \%, 28.57 \%$, and $14.29 \%$, respectively and $57.14 \%, 14.29 \%$, and $28.57 \%$, respectively, for the third lesson plan. Results of the analysis of the lesson plans related to this structure indicate participants did develop in their ability to design lessons that include instructional strategies that promote retention of ideas in their students. However, the growth was not as substantial as in the first interaction structure where $50 \%$ of the third lesson plans were considered high.

For the third interaction structure, the percent of low, medium, and high first lesson plans were $50 \%, 30 \%$, and $20 \%$, respectively. The percent of low, medium, and high second lesson plans were $50 \%, 40 \%$, and $10 \%$, respectively and the percent of low, medium, and high third lesson plans were $40 \%, 40 \%$, and $20 \%$, respectively. Results of the analysis in this interaction structure indicate participants grew very little in their ability to design lessons that include instructional strategies that promote retention of ideas in their students.

Comparison of the results from all interaction structures for Graeber's fourth big idea suggest that the most growth in elementary preservice teachers' ability to design lessons that include instructional strategies that promote retention amongst their students occurred in the first interaction structure. Although, some growth did occur in the second interaction structure, it would elementary preservice teachers benefit from the added support of the MLS mentor during the second debriefing session. 
Big idea \#5: Alternative representations and the recognition and analysis of alternative methods are important

For Graeber's fifth big idea, alternative representations and the recognition and analysis of alternative methods are important, the percent of lessons coded as low, medium, and high for each lesson in each interaction structure was calculated, see Table 9. A graphical representation of these results can also be found in Figure 5.

Table 9

Percentage of Low, Medium, and High Lessons per Interaction Structure for Big Idea \#5: Alternative representations and the recognition and analysis of alternative methods are important

\begin{tabular}{|l|r|r|r|r|r|r|r|r|r|}
\hline & \multicolumn{3}{|c|}{ Interaction Structure \#1 } & \multicolumn{2}{c|}{ Interaction Structure \#2 } & \multicolumn{3}{c|}{ Interaction Structure \#3 } \\
\hline & $\begin{array}{l}\text { Lesson } \\
\text { Plan \#1 }\end{array}$ & $\begin{array}{l}\text { Lesson } \\
\text { Plan \#2 }\end{array}$ & $\begin{array}{l}\text { Lesson } \\
\text { Plan \#3 }\end{array}$ & $\begin{array}{l}\text { Lesson } \\
\text { Plan \#1 }\end{array}$ & $\begin{array}{l}\text { Lesson } \\
\text { Plan \#2 }\end{array}$ & $\begin{array}{l}\text { Lesson } \\
\text { Plan \#3 }\end{array}$ & $\begin{array}{l}\text { Lesson } \\
\text { Plan \#1 }\end{array}$ & $\begin{array}{l}\text { Lesson } \\
\text { Plan \#2 }\end{array}$ & $\begin{array}{l}\text { Lesson } \\
\text { Plan \#3 }\end{array}$ \\
\hline Low & 0 & 12.5 & 0.00 & 14.29 & 14.29 & 14.29 & 10 & 0 & 0.00 \\
\hline Medium & 37.5 & 37.5 & 25.00 & 42.86 & 28.57 & 57.14 & 40 & 50 & 40.00 \\
\hline High & 62.5 & 50 & 75.00 & 42.86 & 57.14 & 28.57 & 50 & 50 & 60.00 \\
\hline
\end{tabular}

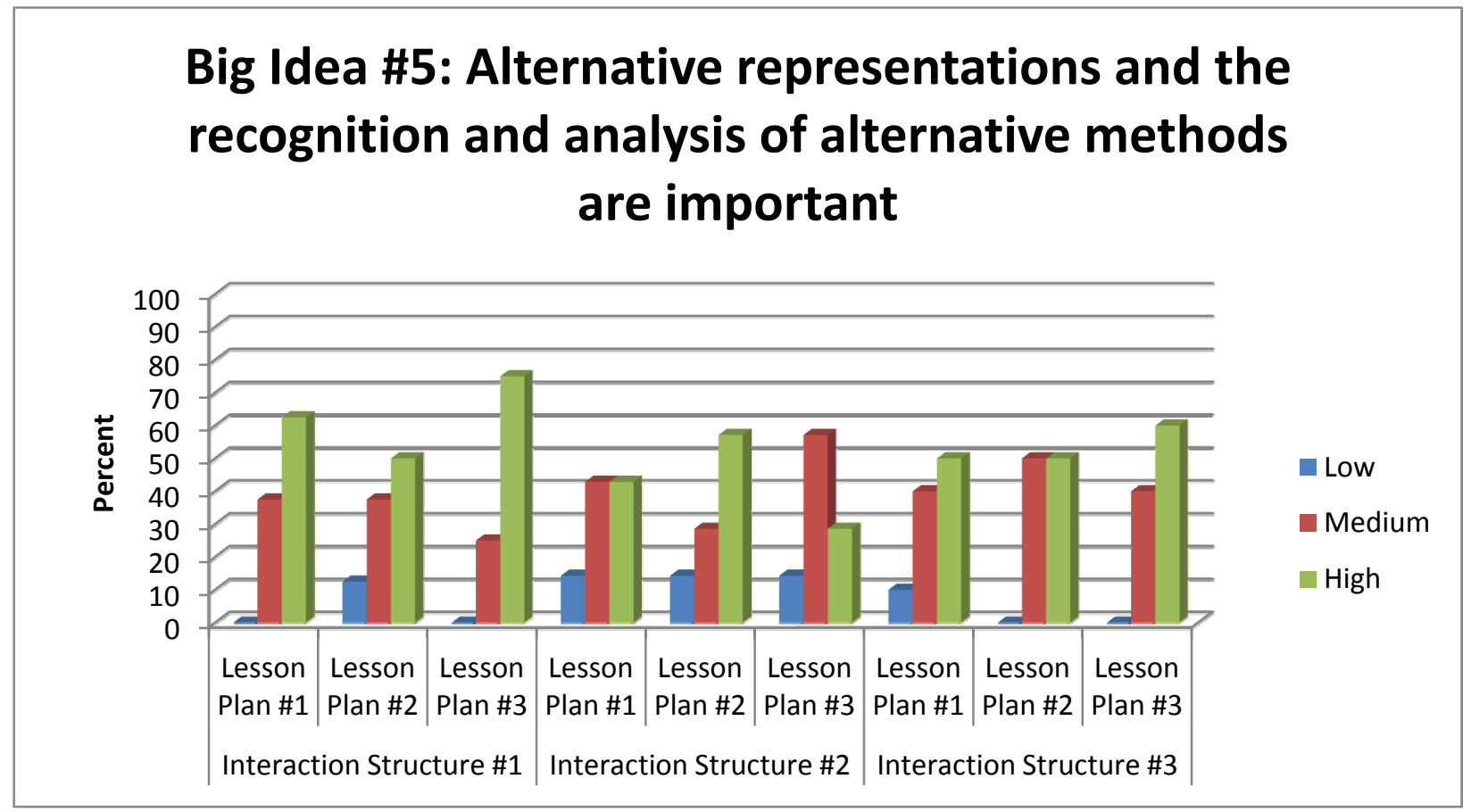

Figure 5. Graph of Growth in Graeber's Big Idea \#5: Alternative representations and the recognition and analysis of alternative methods are important. 
For the first interaction structure, the percentage of first lesson plans classified as low, medium, and high were $0 \%, 37.5 \%$, and $62.5 \%$, respectively. The percent of low, medium, and high second lesson plans were $12.5 \%, 37.5 \%$, and $50 \%$, respectively and the percent of low, medium, and high third lesson plans were $0 \%, 25 \%$, and $75 \%$, respectively. While participants in this interaction structure did grow from the first to the third lesson plan, the growth was not as substantial as in other aspects of Graeber's framework.

For the second interaction structure, the percent of low, medium, and high first lesson plans were $14.29 \%, 42.86 \%$, and $42.86 \%$, respectively. The percent of low, medium, and high second lesson plans were $14.29 \%, 28.57 \%$, and $57.14 \%$, respectively and $14.29 \%, 57.14 \%$, and $28.57 \%$, respectively, for the third lesson plan. Results of the analysis of the lesson plans related to this structure indicate participants actually regressed in their ability to include alternative representations in their lessons as the percentage of high lessons dropped from $42.86 \%$ to $28.57 \%$ in the third lesson. Again, this suggests that elementary preservice teachers may need the added support of the MLS mentor during the second debriefing session in order to prevent regression in aspects of their pedagogical content knowledge.

For the third interaction structure, the percent of low, medium, and high first lesson plans were $10 \%, 40 \%$, and $50 \%$, respectively. The percent of low, medium, and high second lesson plans were $0 \%, 50 \%$, and $50 \%$, respectively and the percent of low, medium, and high third lesson plans were $0 \%, 40 \%$, and $60 \%$, respectively. Results of the analysis in this interaction structure indicate participants grew very little in their ability to design lessons that include alternative representations of a concept. However, it is important to note that participants in this interaction structure did not regress in the ability to design lessons in line with Graeber's fifth big idea. 
Results of the analysis of all three interaction structures for Graeber's fifth big idea suggest that elementary preservice teachers' ability to design lessons that include alternative representations of a concept increased most in the first interaction structure. Interestingly, elementary preservice teachers actually regressed in their ability to include alternative representations in their lesson plans during the second interaction structure. This would indicate that elementary preservice teachers require the added support of the MLS mentor during the second debriefing session in order to prevent regression in aspects of their pedagogical content knowledge.

\section{Discourse Analysis}

A total of 12 MLS groups were selected for discourse analysis. This included four groups from each interaction structure. As discussed previously, groups were selected for analysis based on results of the pre and post assessment used for the content analysis.

Descriptive codes were created for first-level coding, see Appendix F, and analyzed for patterns and themes. Four themes arose from the pattern analysis and included: (1) Content Knowledge Discussions, (2) Pedagogical Content Knowledge Discussions, (3) MLS Group Issues, and (4) Lesson Planning within the MLS Interaction Structure, see Appendix G. The percent of words related to each theme were used to create bar graphs to visually represent the results.

Additionally, bar graphs were created to visually represent the pattern codes for the Pedagogical Content Knowledge Discussions. These codes were Graeber's (1999) five big ideas and included the following: (1) “Understanding students' current understanding” (p.192), (2) "Students knowing in one way do not necessarily know in the other(s)" (p.195), (3) "Intuitive knowledge is both an asset and a liability" (p.198), (4) "Certain instructional characteristics 
appear to promote retention" (p.200), (5) "Alternative representations and the recognition and analysis of alternative methods are important" (p.202).

\section{Interaction Structure \#1}

For the first interaction structure, in which the MLS mentor participated in the first and second debriefing sessions, the two groups with the highest total gains from the second preassessment to the post-assessment were Properties of Quadrilaterals (Course Section \#2) with a $33.3 \%$ overall group gain and Properties of Quadrilaterals (Course Section \#1) with a 22.2\% overall group gain. The two groups with the least total gains from the second pre-assessment to the post-assessment were Adding Fractions (Course Section \#1) with a -33.3\% overall group gain and Adding Fractions (Course Section \#2) with a 0\% overall group gain.

\section{Groups with Highest Total Gains}

For the Properties of Quadrilaterals group (Course Section \#2), with an overall group content gain of $33.3 \%$, the percent of total words for each theme for each of the debriefing sessions were calculated, see Table 10 and Figure 6 . For each of the themes, the percent of total words is given in order of debriefing session as follows: Content Knowledge Discussions, 15.568\%, 15.491\%, 11.345\%; Pedagogical Content Knowledge Discussions, 19.242\%, 6.08\%, 0\%; MLS Group Issues, 3.382\%, 2.48\%, 0\%; Lesson Planning within MLS Structure, 61.808\%, $75.952 \%, 88.655 \%$. Most notable is the greatest percentage of words for all the debriefing sessions were used to discuss lesson planning within MLS structure. Also, the least percentage of words were used to discuss MLS group issues during the first two debriefing sessions. No discussion occurred in the third debriefing session related to this theme, which may indicate the presence of the MLS mentor could have served to clarify or mediate any initial group issues. 
Table 10

Percentage of words per theme for debriefing sessions with Properties of Quadrilaterals (Course Section \#2) group

\begin{tabular}{|c|r|r|r|}
\hline Theme & Debrief \#1 & \multicolumn{1}{l|}{ Debrief \#2 } & Debrief \#3 \\
\hline Content Knowledge Discussions & 15.568 & 15.491 & 11.345 \\
\hline Pedagogical Content Knowledge Discussions & 19.242 & 6.08 & 0 \\
\hline MLS Group Issues & 3.382 & 2.48 & 0 \\
\hline Lesson Planning within MLS Structure & 61.808 & 75.952 & 88.655 \\
\hline
\end{tabular}

\section{Properties of Quadrilaterals (Course Section \#2)}

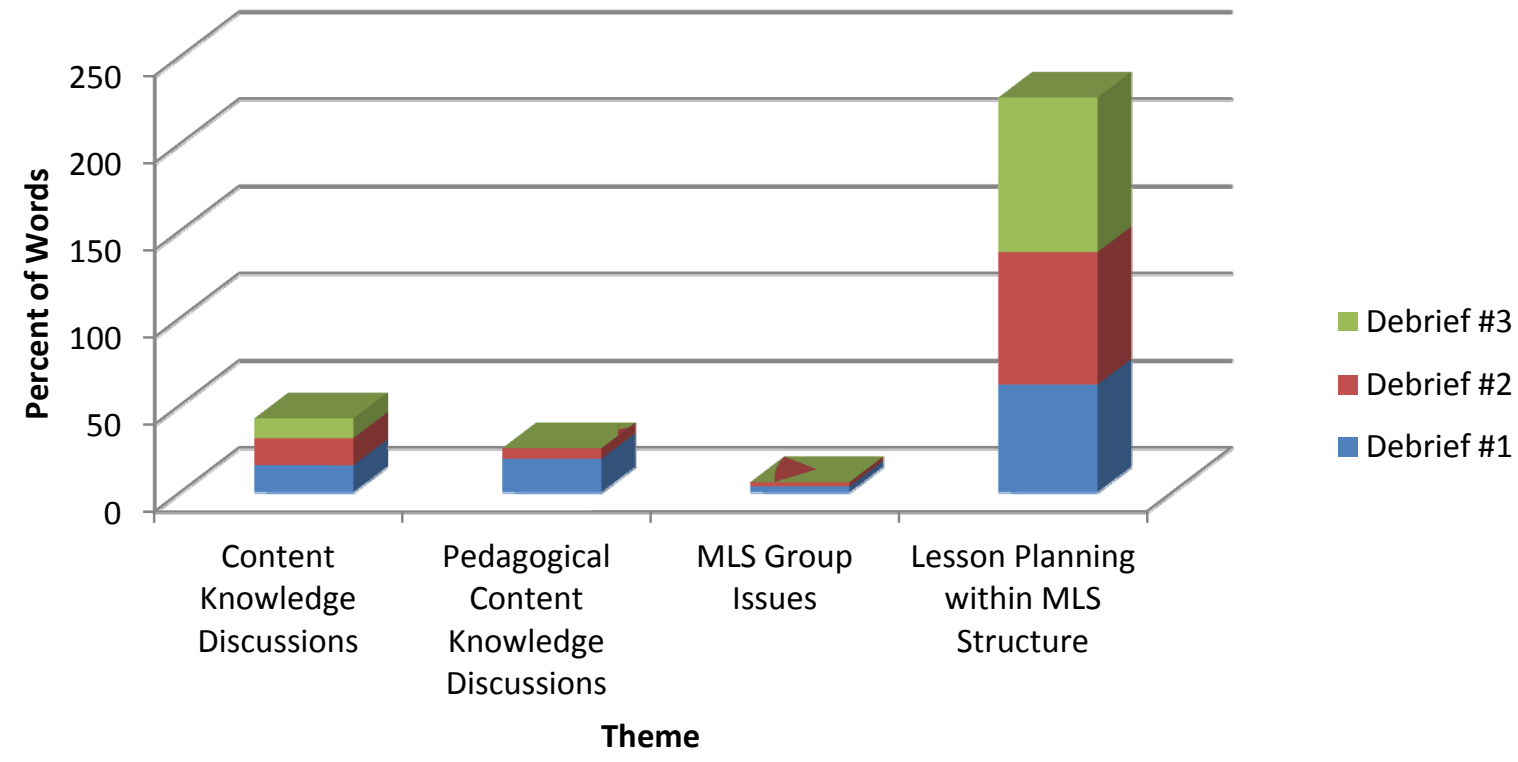

Figure 6. Bar graph of percent of words per theme for each debriefing session for Properties of Quadrilaterals (Course Section \#2) group.

The percent of total words for each of Graeber's (1999) big ideas within the Pedagogical Content Knowledge Discussions for each of the debriefing sessions were calculated for the Properties of Quadrilaterals (Course Section \#2) group, see Table 11 and Figure 7. It should be noted that the percents presented are out of the total percent of words for each debriefing session. For each of the big ideas, the percent of total words is given in order of debriefing session as 
follows: Understanding students' current understanding, $19.242 \%, 0 \%, 0 \%$; Students knowing in one way do not necessarily know in the other(s), $0 \%, 0 \%, 0 \%$; Intuitive knowledge is both an asset and a liability, $0 \%, 0 \%, 0 \%$; Certain instructional characteristics appear to promote retention, $0 \%, 6.08 \%, 0 \%$; Alternative representations and the recognition and analysis of alternative methods are important, $0 \%, 0 \%, 0 \%$. This indicates all the discussions related to Pedagogical Content Knowledge for the first debriefing session were related to understanding students' current understanding and for the second debriefing session, certain instructional characteristics that appear to promote retention were the focus of the discussions.

Table 11

Percentage of words per Graeber's (1999) Big Ideas for Pedagogical Content Knowledge Discussions theme with Properties of Quadrilaterals (Course Section \#2) group

\begin{tabular}{|c|c|c|c|}
\hline Pedagogical Content Knowledge: Big Ideas & Debrief \#1 & Debrief \#2 & Debrief \#3 \\
\hline Understanding students' current understanding & 19.242 & 0 & 0 \\
\hline Students knowing in one way do not necessarily know in the other(s) & 0 & 0 & 0 \\
\hline Intuitive knowledge is both an asset and a liability & 0 & 0 & 0 \\
\hline Certain instructional characteristics appear to promote retention & 0 & 6.08 & 0 \\
\hline $\begin{array}{l}\text { Alternative representations and the recognition and analysis of } \\
\text { alternative methods are important }\end{array}$ & 0 & 0 & 0 \\
\hline
\end{tabular}




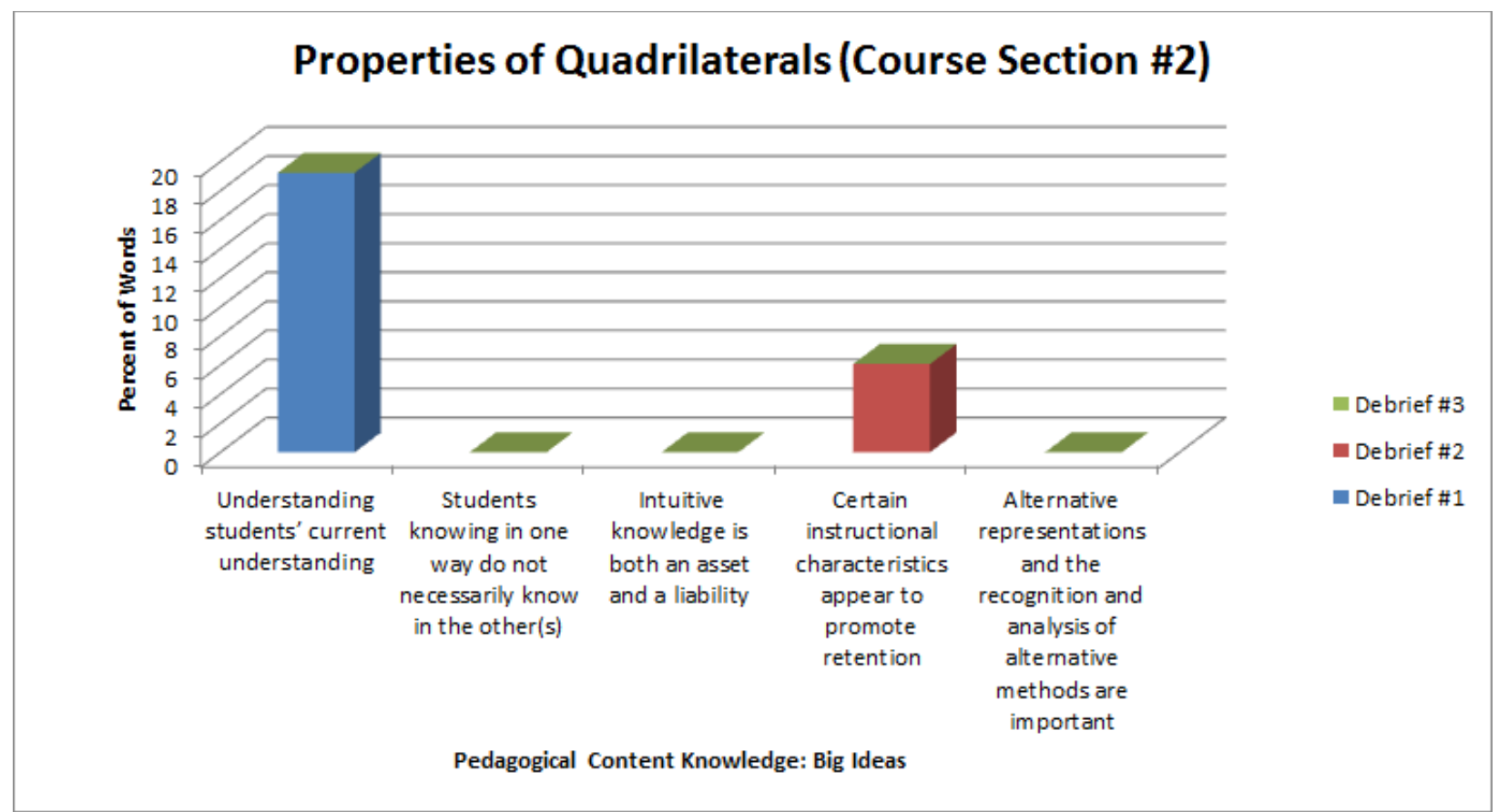

Figure 7. Bar graph of percent of words per Graeber's (1999) Big Idea for Pedagogical Content Knowledge Discussions theme for Properties of Quadrilaterals (Course Section \#2) group.

For the Properties of Quadrilaterals group (Course Section \#1), with an overall group content gain of $22.2 \%$, the percent of total words for each theme for each of the debriefing sessions were calculated, see Table 12 and Figure 8 . For each of the themes, the percent of total words is given in order of debriefing session as follows: Content Knowledge Discussions, 0\%, 3.928\%, 0\%; Pedagogical Content Knowledge Discussions, 20.458\%, 70.873\%, 65.759\%; MLS Group Issues, 0\%, 0\%, 0\%; Lesson Planning within MLS Structure, 79.541\%, 25.199\%, $26.757 \%$. Similar to the other properties of quadrilateral group, this group also spent the majority of the first debriefing session discussing lesson planning within the MLS structure with the rest of the discussions spent on pedagogical content knowledge. However, in the second debriefing session, the distribution of the discussions switches to the most discussions spent on pedagogical content knowledge followed by lesson planning, and some minor discussions related to content knowledge. The distribution of the third debriefing session modeled the second debriefing session distribution in that the most discussions were related to pedagogical content 
knowledge followed by lesson planning within the MLS structure with no discussions related to content knowledge. For all three debriefing sessions, no discussion related to MLS group issues occurred.

Table 12

Percentage of words per theme for debriefing sessions with Properties of Quadrilaterals (Course Section \#1) group

\begin{tabular}{|c|r|r|r|}
\hline Theme & Debrief \#1 & Debrief \#2 & Debrief \#3 \\
\hline Content Knowledge Discussions & 0 & 3.928 & 0 \\
\hline Pedagogical Content Knowledge Discussions & 20.458 & 70.873 & 65.759 \\
\hline MLS Group Issues & 0 & 0 & 0 \\
\hline Lesson Planning within MLS Structure & 79.541 & 25.199 & 26.757 \\
\hline
\end{tabular}

\section{Properties of Quadrilaterals (Course Section \#1)}

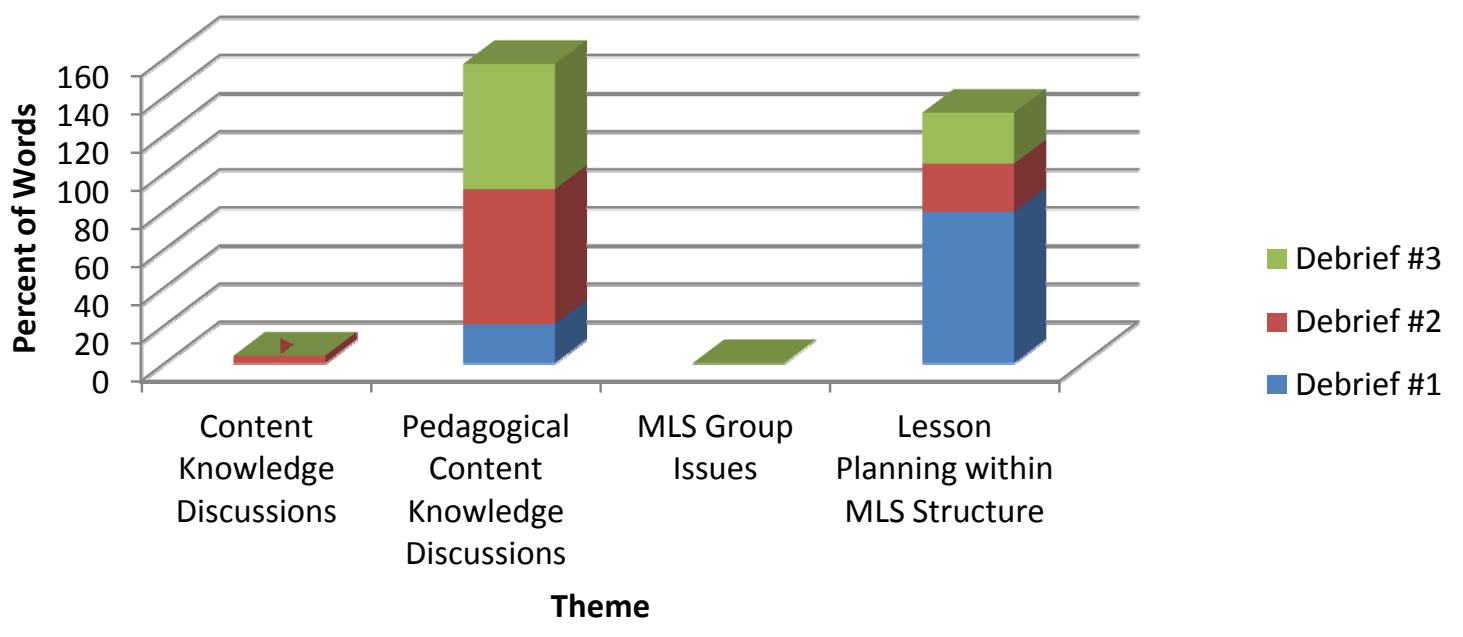

Figure 8. Bar graph of percent of words per theme for each debriefing session for Properties of Quadrilaterals (Course Section \#1) group.

The percent of total words for each of Graeber's (1999) big ideas within the Pedagogical Content Knowledge Discussions for each of the debriefing sessions were calculated for the Properties of Quadrilaterals (Instructor \#1) group, see Table 13 and Figure 9. For each of the big ideas, the percent of totals words is given in order of debriefing session as follows: 
Understanding students' current understanding, 6.419\%, 42.103\%, 22.222\%; Students knowing in one way do not necessarily know in the other(s), $0 \%, 0 \%, 0 \%$; Intuitive knowledge is both an asset and a liability, $0 \%, 0 \%, 0 \%$; Certain instructional characteristics appear to promote retention, $14.039 \%, 0 \%, 43.537 \%$; Alternative representations and the recognition and analysis of alternative methods are important, $0 \%, 28.77 \%, 0 \%$. For this group, most of the discussions related to Pedagogical Content Knowledge for the first and third debriefing sessions were related to certain instructional characteristics that appear to promote retention, while the focus of the second debriefing session discussions was understanding students' current understanding. Also, notable is the remaining discussions related to Pedagogical Content Knowledge for the first and third debriefing sessions was understanding students' current understanding, but for the second debriefing session the remaining discussion was related to alternative representations and the recognition and analysis of alternative methods are important. No discussions, for any of the debriefing sessions, involved students knowing in one way do not necessarily know in the other(s) or intuitive knowledge is both an asset and a liability.

Table 13

Percentage of words per Graeber's (1999) Big Ideas for Pedagogical Content Knowledge Discussions theme with Properties of Quadrilaterals (Course Section \#1) group

\begin{tabular}{|c|r|r|r|}
\hline Pedagogical Content Knowledge: Big Ideas & Debrief \#1 & Debrief \#2 & Debrief \#3 \\
\hline $\begin{array}{c}\text { Understanding students' current understanding } \\
\text { other(s) }\end{array}$ & 6.419 & 42.103 & 22.222 \\
\hline $\begin{array}{r}\text { Intuitive knowledge is both an asset and a liability } \\
\text { retention }\end{array}$ & 0 & 0 & 0 \\
\hline $\begin{array}{r}\text { Certain instructional characteristics appear to promote } \\
\text { Alternative representations and the recognition and analysis of } \\
\text { alternative methods are important }\end{array}$ & 14.039 & 0 & 43.537 \\
\hline
\end{tabular}




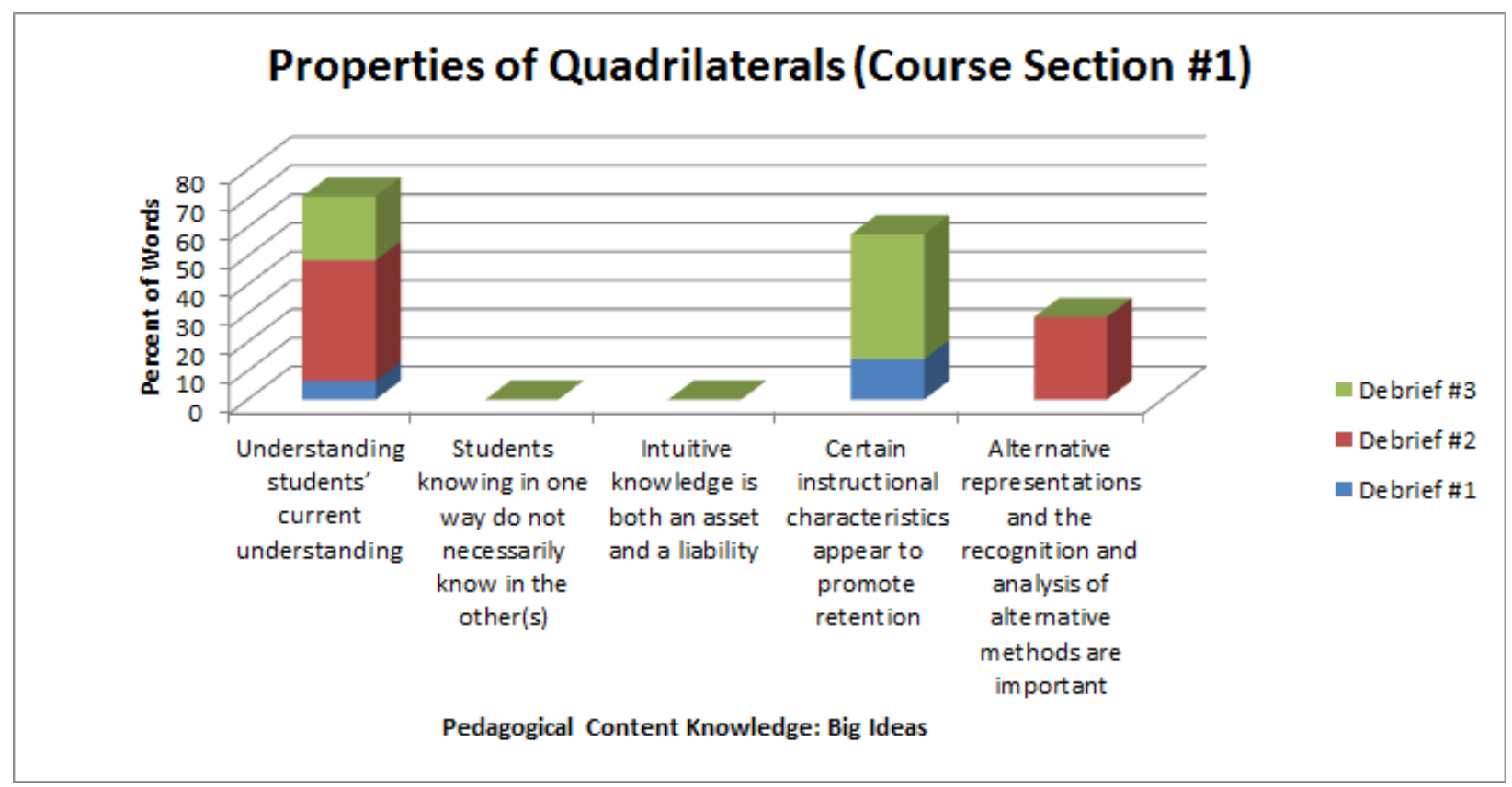

Figure 9. Bar graph of percent of words per Graeber's (1999) Big Ideas for Pedagogical Content Knowledge Discussions theme for Properties of Quadrilaterals (Course Section \#1) group.

\section{Groups with Least Total Gains}

For the Adding Fractions (Course Section \#1), with an overall group content gain of $33.3 \%$, the percent of total words for each theme for each of the debriefing sessions were calculated, see Table 14 and Figure 10. For each of the themes, the percent of total words is given in order of debriefing session as follows: Content Knowledge Discussions, 10.245\%, 10.974\%, 0\%; Pedagogical Content Knowledge Discussions, 37.029\%, 64.274\%, 60.736\%; MLS Group Issues, 0\%, 0\%, 0\%; Lesson Planning within MLS Structure, 52.726\%, 24.753\%, 39.264\%. Again, for the first debriefing session, the greatest percent of words were for discussions related to lesson planning within MLS structure followed by Pedagogical Content Knowledge discussions and then Content Knowledge discussions. However, for the second and third debriefing sessions, Pedagogical Content Knowledge was discussed the most followed by lesson planning within the MLS structure and for the second debriefing session, the least 
discussion related to Content Knowledge. For all three debriefing sessions, no discussion related to MLS group issues occurred.

Table 14

Percentage of words per theme for debriefing sessions with Adding Fractions (Course Section \#1) group

\begin{tabular}{|c|r|r|r|}
\hline Theme & Debrief \#1 & Debrief \#2 & Debrief \#3 \\
\hline Content Knowledge Discussions & 10.245 & 10.974 & 0 \\
\hline Pedagogical Content Knowledge Discussions & 37.029 & 64.274 & 60.736 \\
\hline MLS Group Issues & 0 & 0 & 0 \\
\hline Lesson Planning within MLS Structure & 52.726 & 24.753 & 39.264 \\
\hline
\end{tabular}

\section{Adding Fractions (Course Section \#1)}

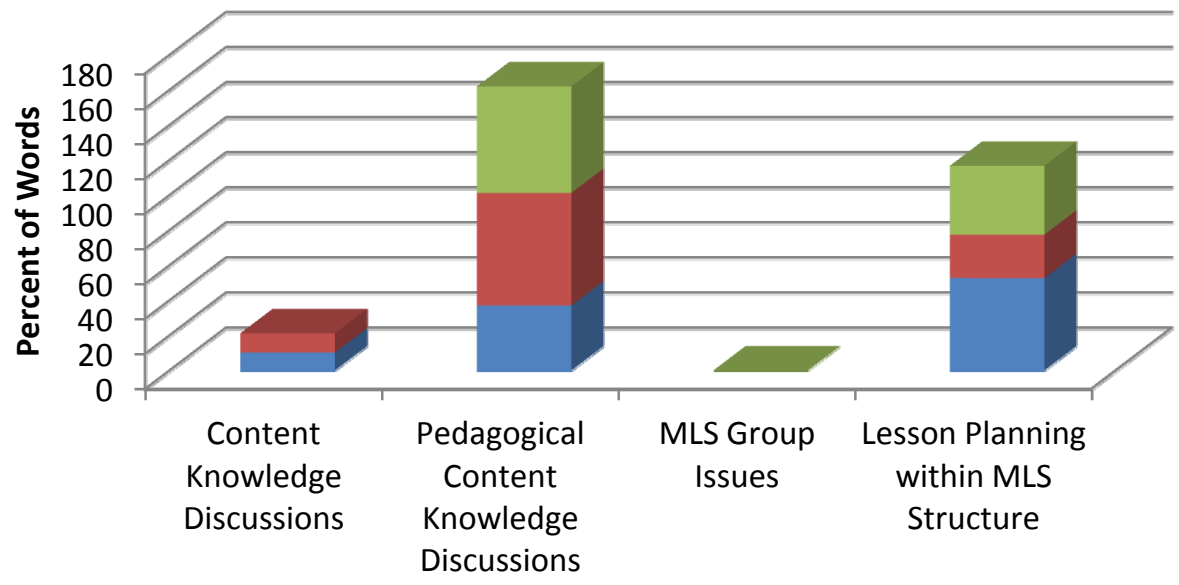

Theme

Figure 10. Bar graph of percent of words per theme for each debriefing session for Adding Fractions (Course Section \#1) group.

The percent of total words for each of Graeber's (1999) big ideas within the Pedagogical Content Knowledge Discussions for each of the debriefing sessions were calculated for the Adding Fractions (Course Section \#1) group, see Table 15 and Figure 11. For each of the big ideas, the percent of total words is given in order of debriefing session as follows: Understanding 
students' current understanding, 18.275\%, 64.274\%, 60.736\%; Students knowing in one way do not necessarily know in the other(s), 0\%, 0\%, 0\%; Intuitive knowledge is both an asset and a liability, $0 \%, 0 \%, 0 \%$; Certain instructional characteristics appear to promote retention, $1.738 \%$, $0 \%, 0 \%$; Alternative representations and the recognition and analysis of alternative methods are important, $17.016 \%, 0 \%, 0 \%$. For this group, most of the discussions related to Pedagogical Content Knowledge for all the debriefing sessions were related to understanding students' current understanding. Also, notable is for the second and third debriefing sessions, understanding students' current understanding, was the only one of Graeber's (1999) five big ideas discussed. Only during the first debriefing session did some discussion related to certain instructional characteristics appear to promote retention and alternative representations and the recognition and analysis of alternative methods are important. No discussions, for any of the debriefing sessions, involved students knowing in one way do not necessarily know in the other(s) or intuitive knowledge is both an asset and a liability.

Table 15

Percentage of words per Graeber's (1999) Big Ideas for Pedagogical Content Knowledge Discussions theme for Adding Fractions (Course Section \#1) group

\begin{tabular}{|c|r|r|r|}
\hline Pedagogical Content Knowledge: Big Ideas & Debrief \#1 & Debrief \#2 & Debrief \#3 \\
\hline $\begin{array}{c}\text { Understanding students' current understanding } \\
\text { Students knowing in one way do not necessarily know in the } \\
\text { other(s) }\end{array}$ & 18.275 & 64.274 & 60.736 \\
\hline $\begin{array}{c}\text { Intuitive knowledge is both an asset and a liability } \\
\text { retention }\end{array}$ & 0 & 0 & 0 \\
\hline $\begin{array}{c}\text { Certain instructional characteristics appear to promote } \\
\text { Alternative representations and the recognition and analysis } \\
\text { of alternative methods are important }\end{array}$ & 1.738 & 0 & 0 \\
\hline
\end{tabular}




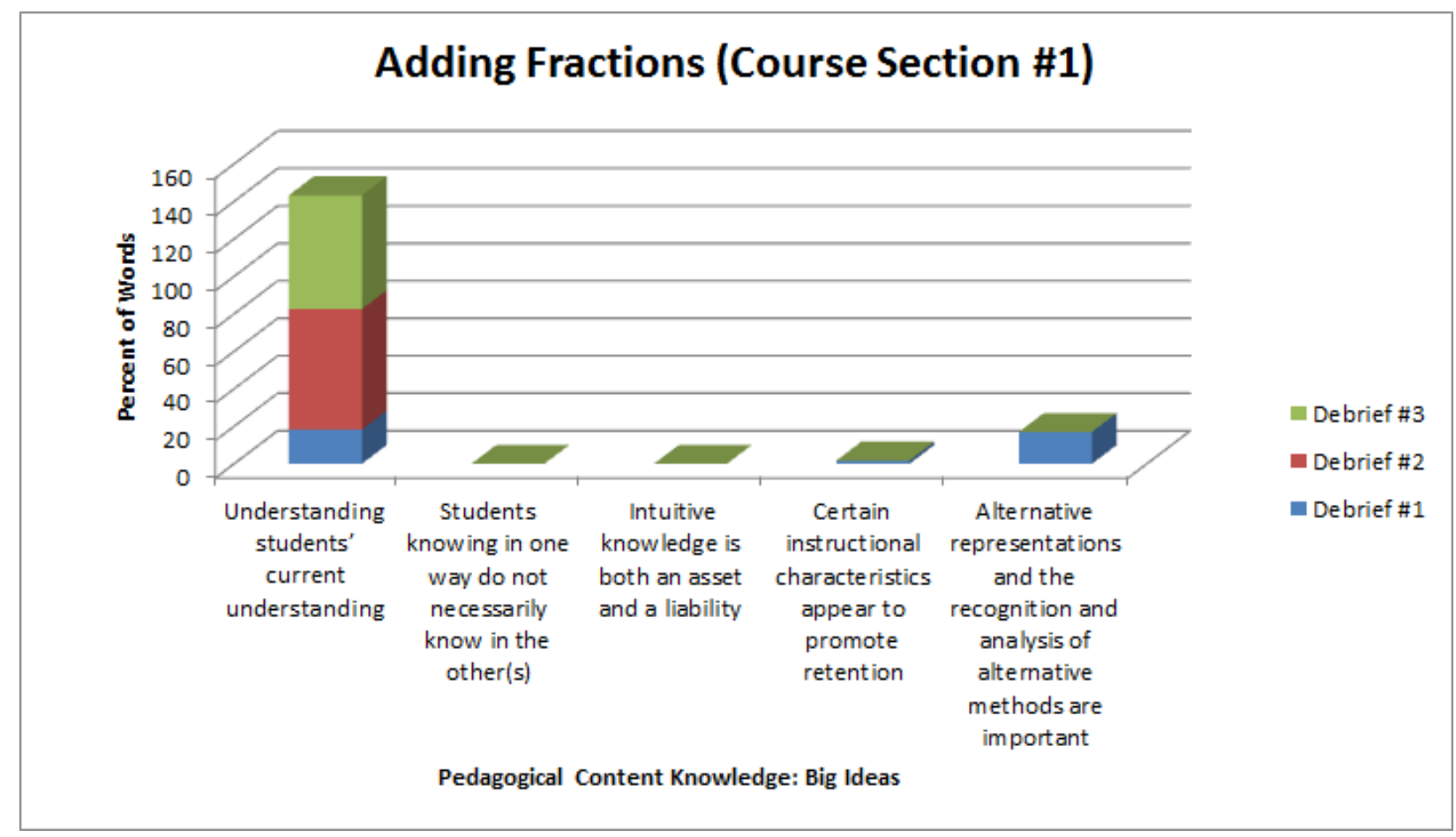

Figure 11. Bar graph of percent of words per Graeber's (1999) Big Ideas for Pedagogical Content Knowledge Discussions theme for Adding Fractions (Course Section \#1) group.

For the Adding Fractions (Course Section \#2), with an overall group content gain of 0\%, the percent of total words for each theme for each of the debriefing sessions were calculated, see Table 16 and Figure 12. For each of the themes, the percent of totals words is given in order of debriefing session as follows: Content Knowledge Discussions, 7.581\%, 11.907\%, 51.109\%; Pedagogical Content Knowledge Discussions, 67.182\%, 59.417\%, 9.6\%; MLS Group Issues, 17.606\%, 6.288\%, 0\%; Lesson Planning within MLS Structure, 7.631\%, 13.64\%, 39.29\%. For this group, the most discussed theme for the first two debriefing sessions was Pedagogical Content Knowledge and for the third debriefing session, the most discussed theme was Content Knowledge. Also, notable is for the first two debriefing sessions, this group had discussions related to MLS group issues, but for the third debriefing session, no discussion was related to this theme. This could indicate that the presence of the MLS mentor during the first two debriefing sessions helped to resolve the MLS group issues. 
Table 16

Percentage of words per theme for debriefing sessions with Adding Fractions (Course Section \#2) group

\begin{tabular}{|c|r|r|r|}
\hline Theme & Debrief \#1 & Debrief \#2 & Debrief \#3 \\
\hline Content Knowledge Discussions & 7.581 & 11.907 & 51.109 \\
\hline Pedagogical Content Knowledge Discussions & 67.182 & 59.417 & 9.6 \\
\hline MLS Group Issues & 17.606 & 6.288 & 0 \\
\hline Lesson Planning within MLS Structure & 7.631 & 13.64 & 39.29 \\
\hline
\end{tabular}

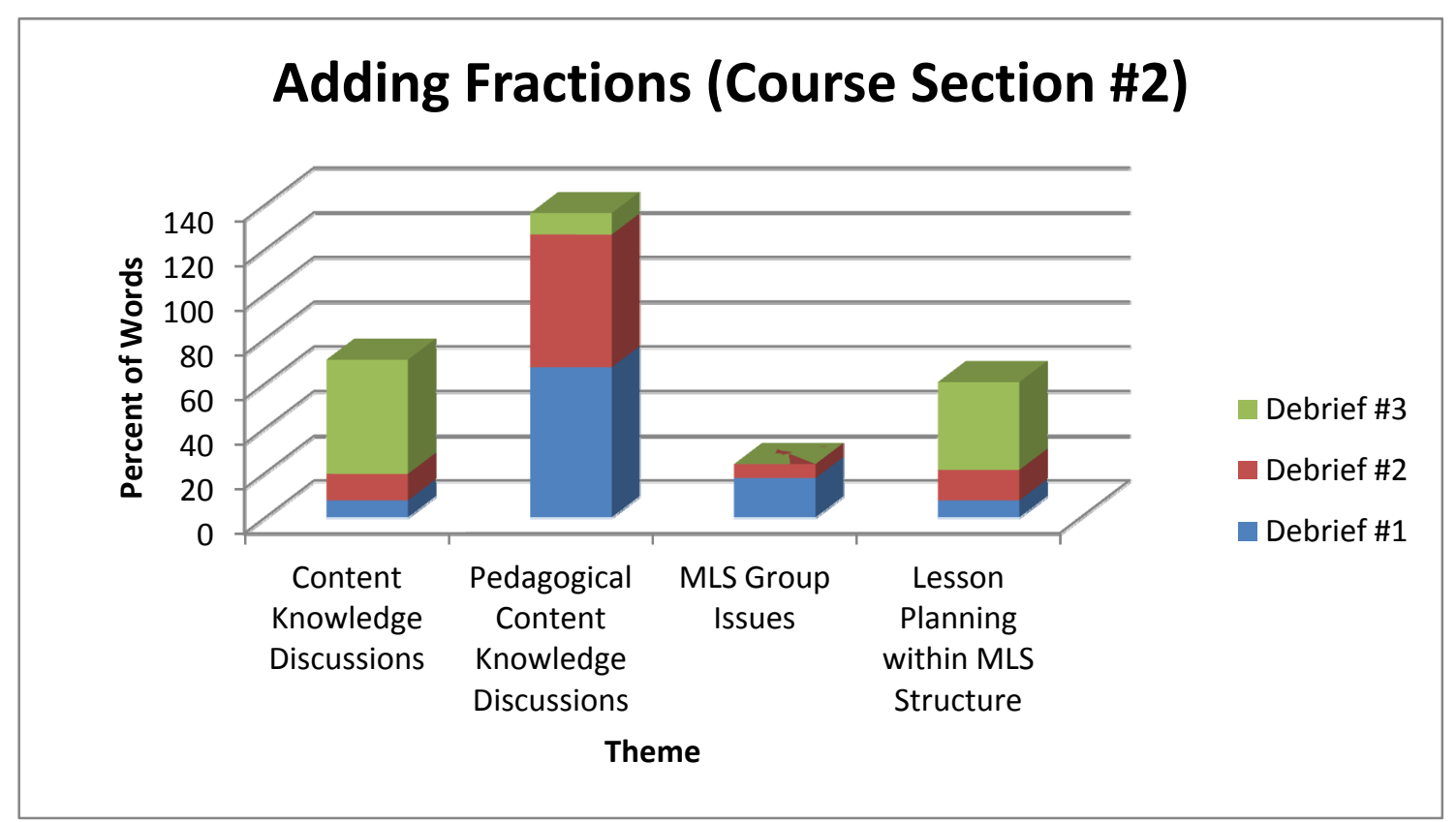

Figure 12. Bar graph of percent of words per theme for each debriefing session for Adding Fractions (Course Section \#2) group.

The percent of total words for each of Graeber's (1999) big ideas within the Pedagogical Content Knowledge Discussions for each of the debriefing sessions were calculated for the Adding Fractions (Course Section \#2) group, see Table 17 and Figure 13. For each of the big ideas, the percent of totals words is given in order of debriefing session as follows:

Understanding students' current understanding, $0 \%, 0 \%, 0 \%$; Students knowing in one way do not necessarily know in the other(s), $0 \%, 0 \%, 0 \%$; Intuitive knowledge is both an asset and a liability, $0 \%, 0.82 \%, 0 \%$; Certain instructional characteristics appear to promote retention, 
$46.782 \%, 40.462 \%, 9.6 \%$; Alternative representations and the recognition and analysis of alternative methods are important, $20.4 \%, 18.135 \%, 0 \%$. For this group, most of the Pedagogical Content Knowledge discussions for all the debriefing sessions were related to certain instructional characteristics appear to promote retention. Also, notable is for the first and second debriefing sessions, alternative representations and the recognition and analysis of alternative methods are important, was the only other of Graeber's (1999) five big ideas discussed. No discussions, for any of the debriefing sessions, involved understanding students' current understanding or students knowing in one way do not necessarily know in the other(s). Minimal discussion related to intuitive knowledge is both an asset and a liability took place in the second debriefing session only.

Table 17

Percentage of words per Graeber's (1999) Big Ideas for Pedagogical Content Knowledge Discussions theme for Adding Fractions (Course Section \#2) group

\begin{tabular}{|c|r|r|r|}
\hline Pedagogical Content Knowledge: Big Ideas & Debrief \#1 & Debrief \#2 & Debrief \#3 \\
\hline $\begin{array}{c}\text { Understanding students' current understanding } \\
\text { other(s) }\end{array}$ & 0 & 0 & 0 \\
\hline $\begin{array}{c}\text { Students knowing in one way do not necessarily know in the } \\
\text { Intuitive knowledge is both an asset and a liability } \\
\text { retention }\end{array}$ & 0 & 0 & 0 \\
\hline $\begin{array}{c}\text { Certain instructional characteristics appear to promote } \\
\begin{array}{c}\text { Alternative representations and the recognition and analysis of } \\
\text { alternative methods are important }\end{array}\end{array}$ & 0 & 0.82 & 0 \\
\hline \multicolumn{2}{|c}{46.782} & 40.462 & 9.6 \\
\hline
\end{tabular}




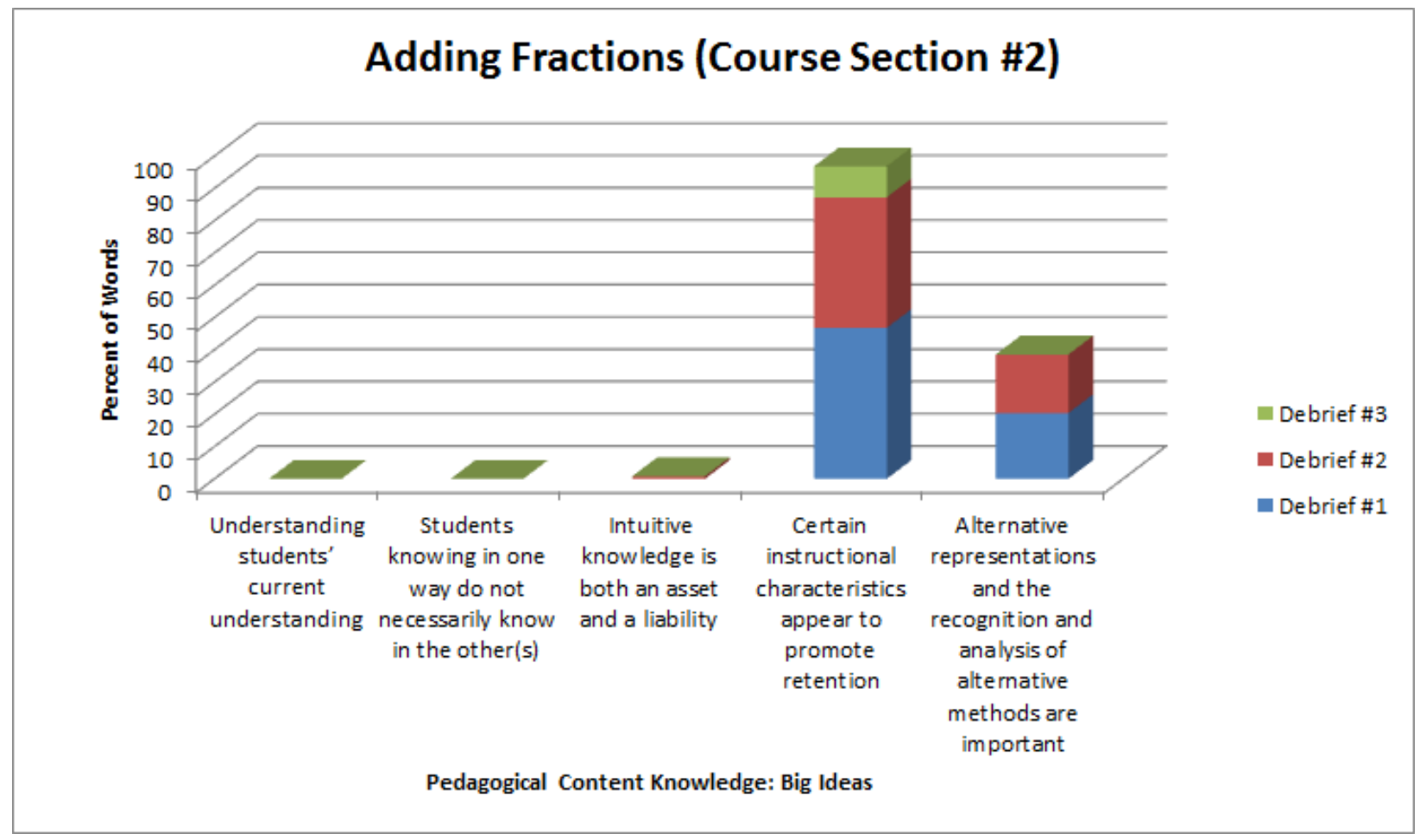

Figure 13. Bar graph of percent of words per Graeber's (1999) Big Ideas for Pedagogical Content Knowledge Discussions theme for Adding Fractions (Course Section \#2) group.

Interestingly, findings from the analysis of the discourse with respect to major themes were similar for all groups in the first interaction structure, which may relate to the presence of the MLS mentor during the first two debriefing sessions. Results from the analysis of the discourse with respect to the content knowledge theme revealed discussions related to the topic were minimal when compared to the other themes, which could indicate the results of the content analysis were dependent on other factors such as the work the group did independent of debriefing sessions. However, pedagogical content knowledge and lesson planning within the MLS structure were heavily discussed throughout the debriefing sessions for all groups, which could explain the growth in pedagogical content knowledge found through the analysis of lesson plans for this structure. 
Further analysis of the pedagogical content knowledge discussions with respect to Graeber's (1999) big ideas demonstrated no group had any discussion during any of the debriefing sessions related to students knowing in one way do not necessarily know in the other(s) or intuitive knowledge is both an asset and a liability. This suggests the MLS mentor may need to make an effort to address these aspects of pedagogical content knowledge during debriefing sessions given that the analysis of the lesson plans for growth with respect to these two big ideas was less when compared to the other three big ideas. Also, with respect to the MLS group issues theme, three of the four groups (the two higher groups and one of the lower groups) had some discussions related to the theme during the first two debriefing sessions, but none during the last debriefing session. This suggests the presence of the MLS mentor during the first two debriefing sessions may have been helpful in resolving MLS group issues as no group had any discussion related to the theme during the last debriefing session.

\section{Interaction Structure \#2}

For the second interaction structure, in which the MLS mentor participated only in the first debriefing session, no group had a negative overall gain for content knowledge as measured. The two groups with the highest total gains from the second pre-assessment to the postassessment were Multiplying Fractions (Course Section \#2) with a 33.3\% overall group gain and Fractions, Decimals, and Percents (Course Section \#1) with a 25\% overall group gain. The two groups with the least overall gain from the second pre-assessment to the post-assessment was the Multiplying Fractions (Course Section \#1) group with a 0\% overall group gain and Addition of two-digit numbers (Course Section \#1) with a $0 \%$ overall gain. 


\section{Groups with Highest Total Gains}

For the Multiplying Fractions (Course Section \#2), with a 33.3\% overall group gain, the percent of total words for each theme for each of the debriefing sessions were calculated, see Table 18 and Figure 14. However, no data was available for the second debriefing session. For each of the themes, the percent of total words is given in order of debriefing session as follows: Content Knowledge Discussions, 30.1\%, 0\%; Pedagogical Content Knowledge Discussions, 12.59\%, 0\%; MLS Group Issues, 3.95\%, 0\%; Lesson Planning within MLS Structure, 53.36\%, $100 \%$. Most notable is the greatest percentage of words for the debriefing sessions available were used to discuss lesson planning within MLS structure. Also, no other theme was discussed in the last debriefing session. The first debriefing session did include discussions related to the other themes with Content Knowledge discussions as the second most discussed theme followed by Pedagogical Content Knowledge discussions, and MLS group issues. It is important to note that no discussion was related to MLS group issues during the last debriefing session, which could indicate the MLS mentor's presence in the first debriefing session was critical.

Table 18

Percentage of words per theme for debriefing sessions with Multiplying Fractions (Course Section \#2) group

\begin{tabular}{|c|r|r|r|}
\hline Theme & Debrief \#1 & Debrief \#2 & \multicolumn{2}{|l|}{ Debrief \#3 } \\
\hline Content Knowledge Discussions & 30.1 & No data & 0 \\
\hline Pedagogical Content Knowledge Discussions & 12.59 & No data & 0 \\
\hline MLS Group Issues & 3.95 & No data & \\
\hline Lesson Planning within MLS Structure & 53.36 & No data & 100 \\
\hline
\end{tabular}




\section{Multiplying Fractions (Course Section \#2)}

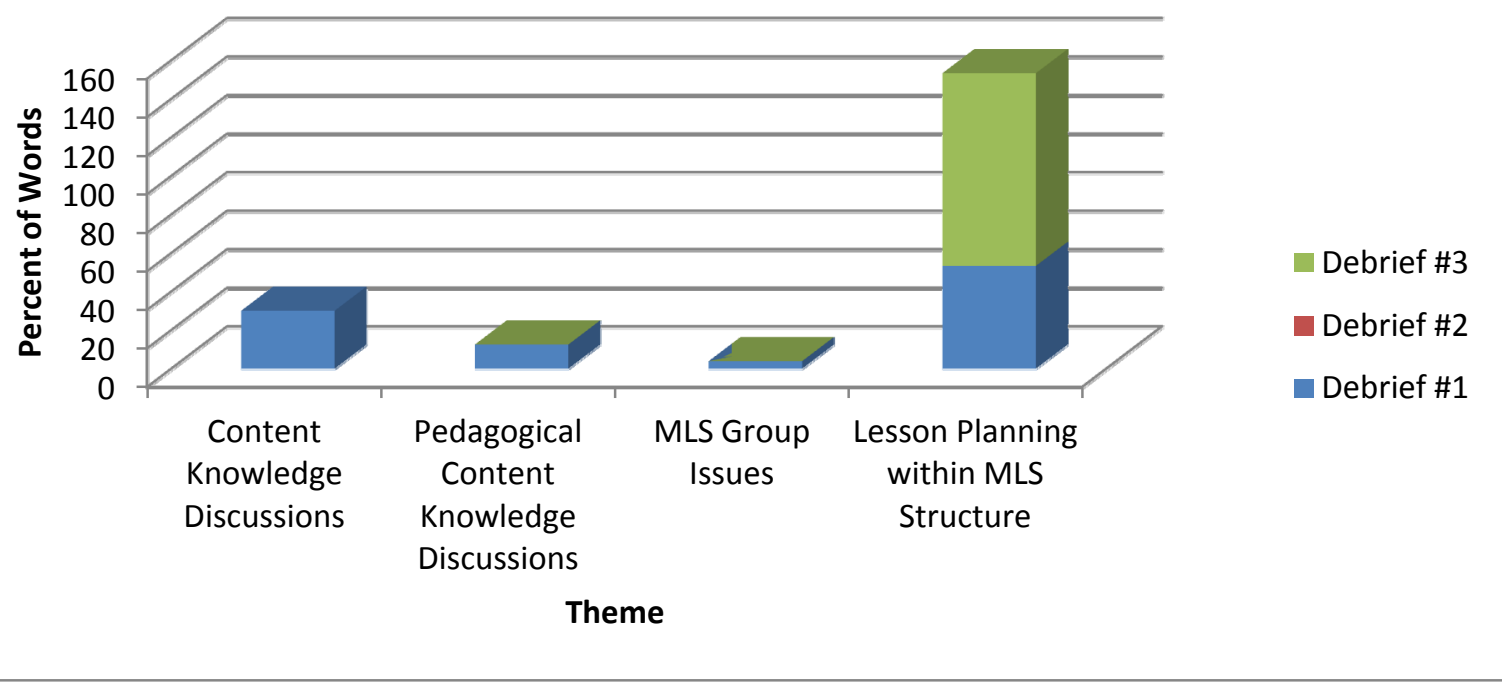

Figure 14. Bar graph of percent of words per theme for each debriefing session for Multiplying Fractions (Course Section \#2) group.

The percent of total words for each of Graeber's (1999) big ideas within the Pedagogical Content Knowledge Discussions for each of the debriefing sessions were calculated for the Multiplying Fractions (Course Section \#2) group, see Table 19 and Figure 15. For each of the big ideas, the percent of totals words is given in order of debriefing session as follows: Understanding students' current understanding, 0\%, 0\%; Students knowing in one way do not necessarily know in the other(s), 0\%,0\%; Intuitive knowledge is both an asset and a liability, $0 \%, 0 \%$; Certain instructional characteristics appear to promote retention, $12.59 \%, 0 \%$;

Alternative representations and the recognition and analysis of alternative methods are important, $0 \%, 0 \%$. For this group, the only Pedagogical Content Knowledge discussion recorded occurred in the first debriefing session and was related to certain instructional characteristics appear to promote retention. No discussions were related to any of Graeber's (1999) other five big ideas. 
Table 19

Percentage of words per Graeber's (1999) Big Ideas for Pedagogical Content Knowledge Discussions theme for Multiplying Fractions (Course Section \#2) group

\begin{tabular}{|c|r|r|r|}
\hline Pedagogical Content Knowledge: Big Ideas & Debrief \#1 & Debrief \#2 & Debrief \#3 \\
\hline $\begin{array}{c}\text { Understanding students' current understanding } \\
\text { other(s) }\end{array}$ & 0 & No data & \\
\hline $\begin{array}{c}\text { Intuitive knowledge is both an asset and a liability } \\
\text { retention }\end{array}$ & 0 & No data & \\
\hline $\begin{array}{c}\text { Certain instructional characteristics appear to promote } \\
\text { Alternative representations and the recognition and analysis of } \\
\text { alternative methods are important }\end{array}$ & 12.59 & No data & \\
\hline
\end{tabular}

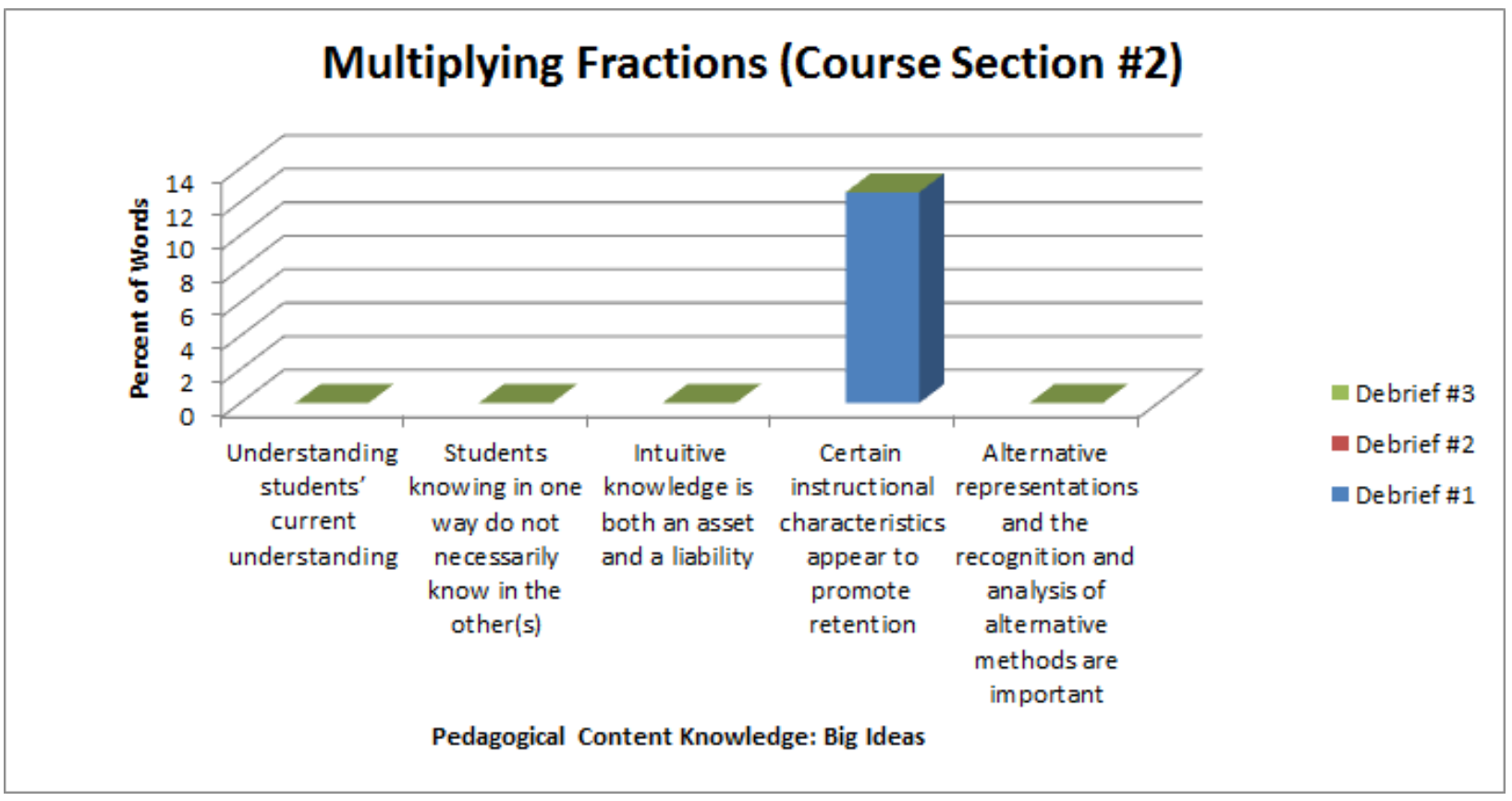

Figure 15. Bar graph of percent of words per Graeber's (1999) Big Ideas for Pedagogical Content Knowledge Discussions theme for Multiplying Fractions (Course Section \#2) group.

For the Fractions, Decimals, and Percents (Course Section \#3), with a 25\% overall group gain, the percent of total words for each theme for each of the debriefing sessions were calculated, see Table 20 and Figure 16. No data was available for the third debriefing session. 
For each of the themes, the percent of totals words is given in order of debriefing session as follows: Content Knowledge Discussions, 29\%, 0\%; Pedagogical Content Knowledge Discussions, 55.329\%, 96.332\%; MLS Group Issues, 0\%, 0\% Lesson Planning within MLS Structure, $28.74 \%, 3.667 \%$. For this group, the greatest percentage of words for the debriefing sessions available were used to discuss pedagogical content knowledge. Interestingly, the first debriefing session, which included the MLS mentor, had the most diverse discussions as the group discussed content knowledge and lesson planning within the MLS structure. During the second debriefing session, for which the MLS mentor was absent, only minimal discussion related to lesson planning within the MLS structure.

Table 20

Percentage of words per theme for debriefing sessions with Fractions, Decimals, and Percents (Course Section \#3) group

\begin{tabular}{|c|r|r|r|}
\hline Theme & Debrief \#1 & Debrief \#2 & Debrief \#3 \\
\hline Content Knowledge Discussions & 29 & 0 & No data \\
\hline Pedagogical Content Knowledge Discussions & 55.329 & 96.332 & No data \\
\hline MLS Group Issues & 0 & 0 & No data \\
\hline Lesson Planning within MLS Structure & 28.74 & 3.667 & No data \\
\hline
\end{tabular}




\section{Fractions, Decimals, and Percents (Course Section \#3)}

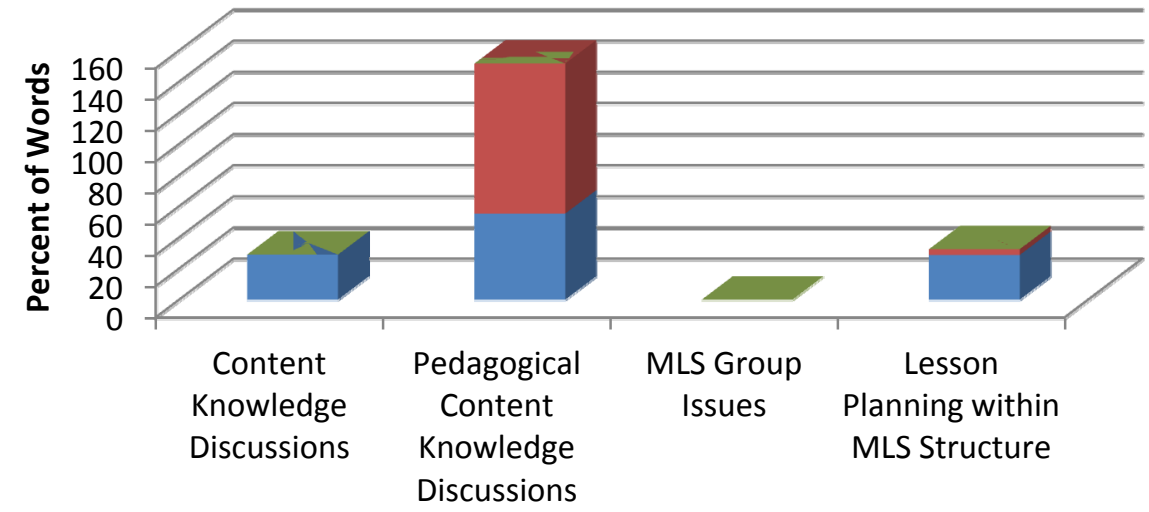

Debrief \#3

Debrief \#2

Debrief \#1

Theme

Figure 16. Bar graph of percent of words per theme for each debriefing session for Fractions, Decimals, and Percents (Course Section \#3) group.

The percent of total words for each of Graeber's (1999) big ideas within the Pedagogical Content Knowledge Discussions for each of the debriefing sessions were calculated for the Multiplying Fractions (Course Section \#2) group, see Table 21 and Figure 17. For each of the big ideas, the percent of totals words is given in order of debriefing session as follows:

Understanding students' current understanding, 0\%, 24.5\%; Students knowing in one way do not necessarily know in the other(s), 0\%, 0\%; Intuitive knowledge is both an asset and a liability, $0 \%, 0 \%$; Certain instructional characteristics appear to promote retention, $39.09 \%, 71.882 \%$;

Alternative representations and the recognition and analysis of alternative methods are important, $16.239 \%, 0 \%$. For this group, certain instructional characteristics appear to promote retention, was discussed most during both debriefing sessions. Alternative representations and the recognition and analysis of alternative methods are important was discussed only during the first debriefing session and understanding students' current understanding was discussed only during 
the second debriefing session. No discussion involved students knowing in one way do not necessarily know in the other(s) and intuitive knowledge is both an asset and a liability.

Table 21

Percentage of words per Graeber's (1999) Big Ideas for Pedagogical Content Knowledge Discussions theme for Fractions, Decimals, and Percents (Course Section \#3) group

\begin{tabular}{|c|r|r|l|}
\hline Pedagogical Content Knowledge: Big Ideas & $\begin{array}{l}\text { Debrief } \\
\# 1\end{array}$ & $\begin{array}{l}\text { Debrief } \\
\# 2\end{array}$ & $\begin{array}{l}\text { Debrief } \\
\# 3\end{array}$ \\
\hline Understanding students' current understanding & 0 & 24.45 & No data \\
\hline Students knowing in one way do not necessarily know in the other(s) & 0 & 0 & No data \\
\hline Intuitive knowledge is both an asset and a liability & 0 & 0 & No data \\
\hline Certain instructional characteristics appear to promote retention & 39.09 & 71.882 & No data \\
\hline $\begin{array}{c}\text { Alternative representations and the recognition and analysis of alternative } \\
\text { methods are important }\end{array}$ & 16.239 & 0 & No data \\
\hline
\end{tabular}

\section{Fractions, Decimals, and Percents (Course Section \#3)}

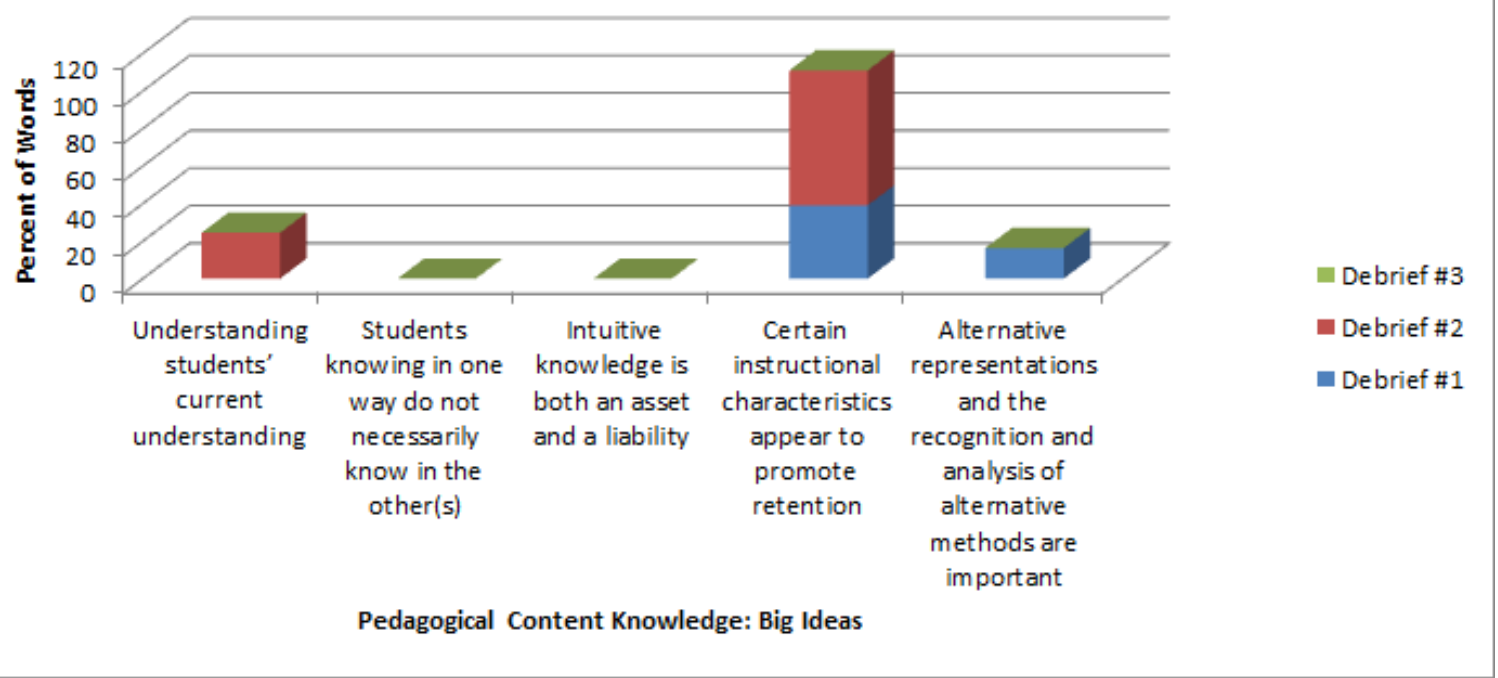

Figure 17. Bar graph of percent of words per Graeber's (1999) Big Ideas for Pedagogical Content Knowledge Discussions theme for Fractions, Decimals, and Percents (Course Section \#3) group. 


\section{Groups with Least Total Gains}

For the Multiplying Fractions (Course Section \#1), with a $0 \%$ overall group gain, the percent of total words for each theme for each of the debriefing sessions were calculated, see Table 22 and Figure 18. However, no data was available for the second debriefing session. For each of the themes, the percent of totals words is given in order of debriefing session as follows: Content Knowledge Discussions, 3.73\%, 27.374, 8.345\%; Pedagogical Content Knowledge Discussions, 39.808\%, 41.036\%, 41.992; MLS Group Issues, 49.78\%, 3.682\%, 0\%; Lesson Planning within MLS Structure, 6.688\%, 27.907\%, 49.664\%. This group appeared to have had a serious group issue as a substantial portion of the first debriefing session involved discussion related to MLS group issues. It would seem the presence of the MLS mentor helped resolve the issues as the second debriefing session involved minimal discussion related to MLS group issues and no discussion during the third debrief session. Aside from the MLS group issues, pedagogical content knowledge appeared to an important theme to this group as a major portion of all three debriefing sessions was spent on the topic. Minor discussions related to content knowledge and lesson planning within the MLS structure were also a part of all three debriefing sessions.

Table 22

Percentage of words per theme for debriefing sessions with Multiplying Fractions (Course Section \#1) group

\begin{tabular}{|c|r|r|r|}
\hline Theme & Debrief \#1 & Debrief \#2 & \multicolumn{2}{|l|}{ Debrief \#3 } \\
\hline Content Knowledge Discussions & 3.73 & 27.374 & 8.345 \\
\hline Pedagogical Content Knowledge Discussions & 39.808 & 41.036 & 41.992 \\
\hline MLS Group Issues & 49.78 & 3.682 & 0 \\
\hline Lesson Planning within MLS Structure & 6.688 & 27.907 & 49.664 \\
\hline
\end{tabular}




\section{Multiplying Fractions (Course Section \#1)}

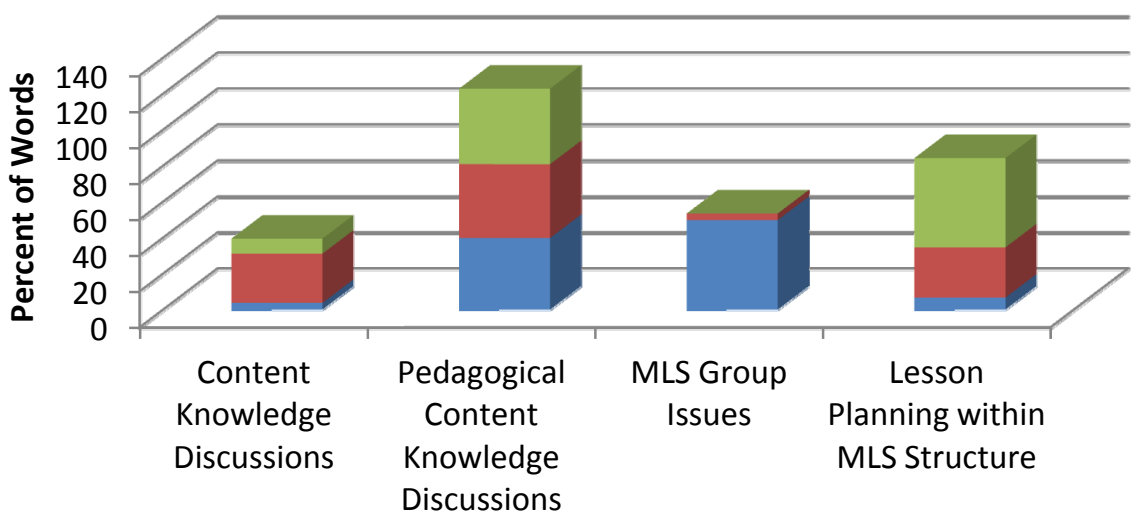

Debrief \#3

Debrief \#2

Debrief \#1

Theme

Figure 18. Bar graph of percent of words per theme for each debriefing session for Multiplying Fractions (Course Section \#1) group.

The percent of total words for each of Graeber's (1999) big ideas within the Pedagogical Content Knowledge Discussions for each of the debriefing sessions were calculated for the Multiplying Fractions (Course Section \#1) group, see Table 23 and Figure 19. For each of the big ideas, the percent of totals words is given in order of debriefing session as follows:

Understanding students' current understanding, 19.41\%, 18.992\%, 8.614\%; Students knowing in one way do not necessarily know in the other(s), $0 \%, 0 \%, 0 \%$; Intuitive knowledge is both an asset and a liability, $0 \%, 0 \%, 0 \%$; Certain instructional characteristics appear to promote retention, $16.01 \%, 10.901 \%, 0 \%$; Alternative representations and the recognition and analysis of alternative methods are important, $4.388 \%, 11.143 \%, 33.378 \%$. For this group, three of Graeber's (1999) big ideas were discussed throughout all the debriefing sessions. Understanding students' current understanding, was discussed most during the first and second debriefing sessions. While alternative representations and the recognition and analysis of alternative methods are important was discussed most during the third debriefing session. Certain instructional characteristics appear to promote retention was discussed during the first and 
second debriefing sessions only. Again no discussion involved students knowing in one way do not necessarily know in the other(s) and intuitive knowledge is both an asset and a liability.

Table 23

Percentage of words per Graeber's (1999) Big Ideas for Pedagogical Content Knowledge Discussions theme for Fractions, Decimals, and Percents (Course Section \#1) group

\begin{tabular}{|c|c|c|c|}
\hline Pedagogical Content Knowledge: Big Ideas & Debrief \#1 & Debrief \#2 & Debrief \#3 \\
\hline Understanding students' current understanding & 19.41 & 18.992 & 8.614 \\
\hline Students knowing in one way do not necessarily know in the other(s) & 0 & 0 & 0 \\
\hline Intuitive knowledge is both an asset and a liability & 0 & 0 & 0 \\
\hline Certain instructional characteristics appear to promote retention & 16.01 & 10.901 & 0 \\
\hline $\begin{array}{l}\text { Alternative representations and the recognition and analysis of } \\
\text { alternative methods are important }\end{array}$ & 4.388 & 11.143 & 33.378 \\
\hline
\end{tabular}

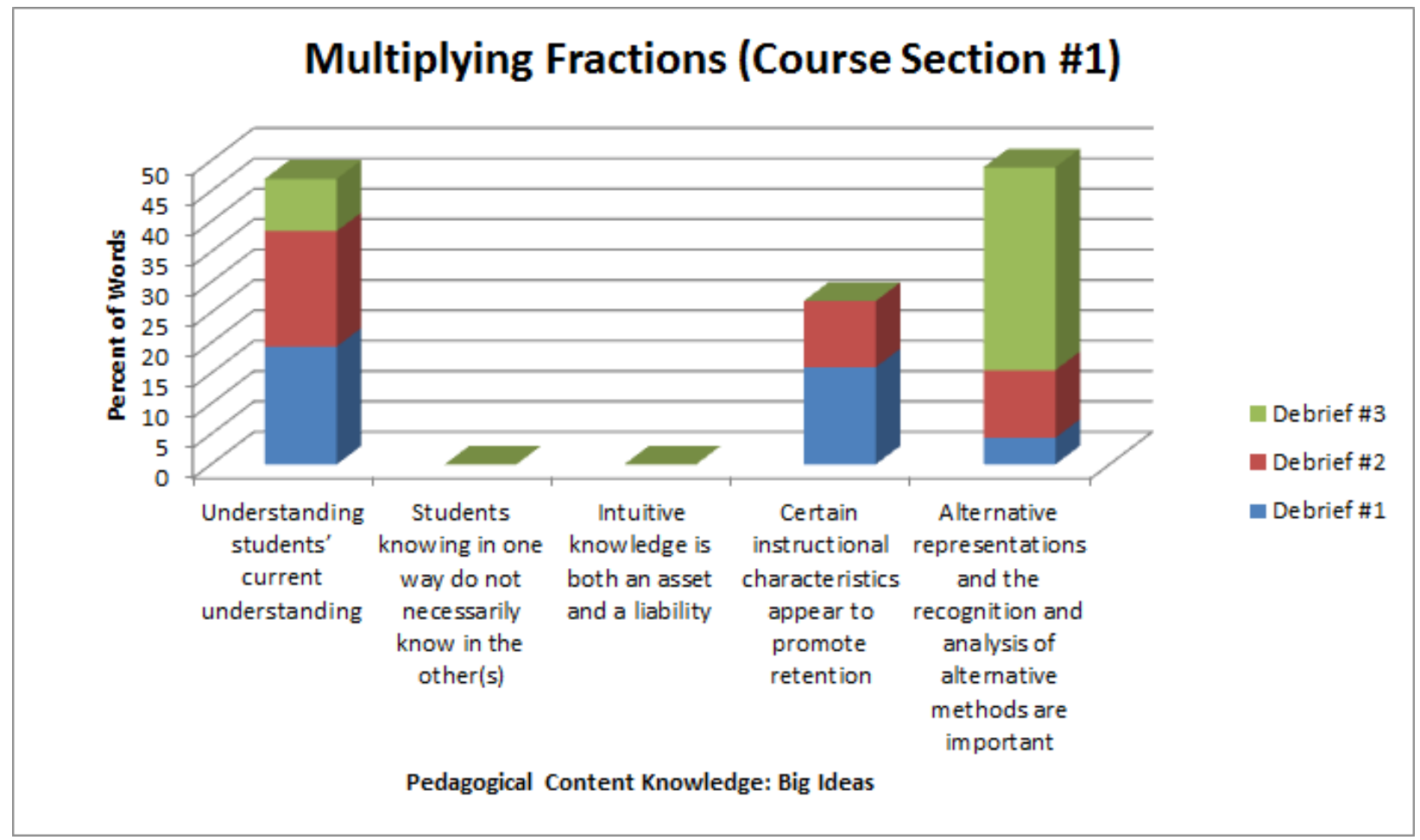

Figure 19. Bar graph of percent of words per Graeber's (1999) Big Ideas for Pedagogical Content Knowledge Discussions theme for Fractions, Decimals, and Percents (Course Section \#3) group. 
For the Addition of two-digit numbers (Course Section \#1), with a $0 \%$ overall group gain, the percent of total words for each theme for each of the debriefing sessions were calculated, see Table 24 and Figure 20. For each of the themes, the percent of totals words is given in order of debriefing session as follows: Content Knowledge Discussions, 6.477\%, 11.86, 0\%; Pedagogical Content Knowledge Discussions, 23.144\%, 49.596\%, 68.323; MLS Group Issues, 0\%, 0\%, 11.947\%; Lesson Planning within MLS Structure, 30.378\%, 38.544\%, 5.188\%. This group's discussions seemed to center around pedagogical content knowledge and lesson planning within the MLS structure discussions throughout the debriefing sessions. Minor discussion related to content knowledge occurred during the first two debriefing sessions. Interestingly, the group appeared to have an issue during the third debriefing session, which could have played a role in the outcome of their MLS experience.

Table 24

Percentage of words per theme for debriefing sessions with Addition of two-digit numbers (Course Section \#1) group

\begin{tabular}{|c|r|r|r|}
\hline Theme & Debrief \#1 & Debrief \#2 & Debrief \#3 \\
\hline Content Knowledge Discussions & 6.477 & 11.86 & 0 \\
\hline Pedagogical Content Knowledge Discussions & 23.144 & 49.596 & 68.323 \\
\hline MLS Group Issues & 0 & 0 & 11.947 \\
\hline Lesson Planning within MLS Structure & 70.378 & 38.544 & 5.188 \\
\hline
\end{tabular}




\section{Addition of two-digit numbers (Course Section \#1)}

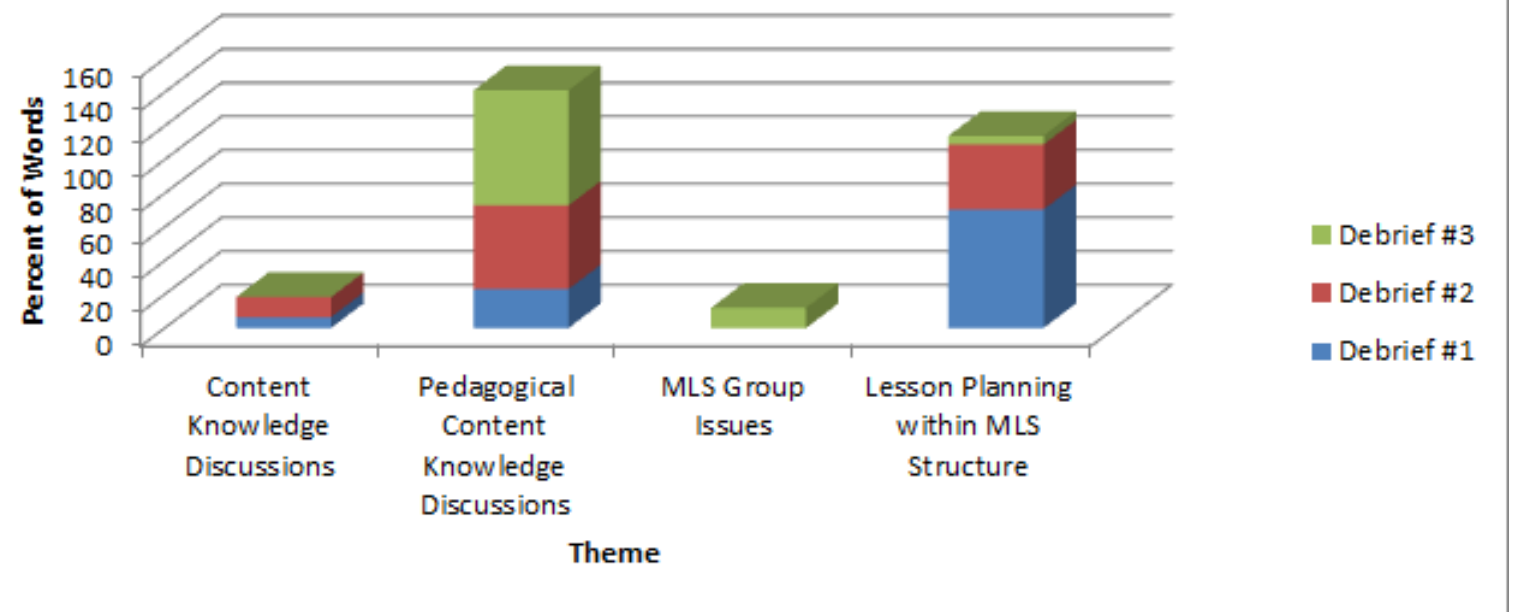

Figure 20. Bar graph of percent of words per theme for each debriefing session for Addition of two-digit numbers (Course Section \#1) group.

The percent of total words for each of Graeber's (1999) big ideas within the Pedagogical Content Knowledge Discussions for each of the debriefing sessions were calculated for the Additon of two-digit numbers (Course Section \#1) group, see Table 25 and Figure 21. For each of the big ideas, the percent of totals words is given in order of debriefing session as follows: Understanding students' current understanding, 9.607\%, 0\%, 0\%; Students knowing in one way do not necessarily know in the other(s), $0 \%, 0 \%, 0 \%$; Intuitive knowledge is both an asset and a liability, $0 \%, 0 \%, 0 \%$; Certain instructional characteristics appear to promote retention, $8.952 \%$, $27.493 \%, 65.349 \%$; Alternative representations and the recognition and analysis of alternative methods are important, $4.585 \%, 22.102 \%, 2.975 \%$. For this group, certain instructional characteristics appear to promote retention and alternative representations and the recognition and analysis of alternative methods are important were the focus of all three debriefing sessions. Some minor discussion related to understanding students' current understanding occurred during the first debriefing session, but no discussion involved students knowing in one way do not necessarily know in the other(s) and intuitive knowledge is both an asset and a liability. 
Table 25

Percentage of words per Graeber's (1999) Big Ideas for Pedagogical Content Knowledge Discussions theme for Addition of two-digit numbers (Course Section \#1) group

\begin{tabular}{|c|r|r|r|}
\hline Pedagogical Content Knowledge: Big Ideas & Debrief \#1 & Debrief \#2 & Debrief \#3 \\
\hline Understanding students' current understanding & 9.607 & 0 & 0 \\
\hline Students knowing in one way do not necessarily know in the other(s) & 0 & 0 & 0 \\
\hline Intuitive knowledge is both an asset and a liability & 0 & 0 & 0 \\
\hline $\begin{array}{r}\text { Certain instructional characteristics appear to promote retention } \\
\text { Alternative representations and the recognition and analysis of }\end{array}$ & 8.952 & 27.493 & 65.349 \\
\hline & 4.585 & 22.102 & 2.975 \\
\hline
\end{tabular}

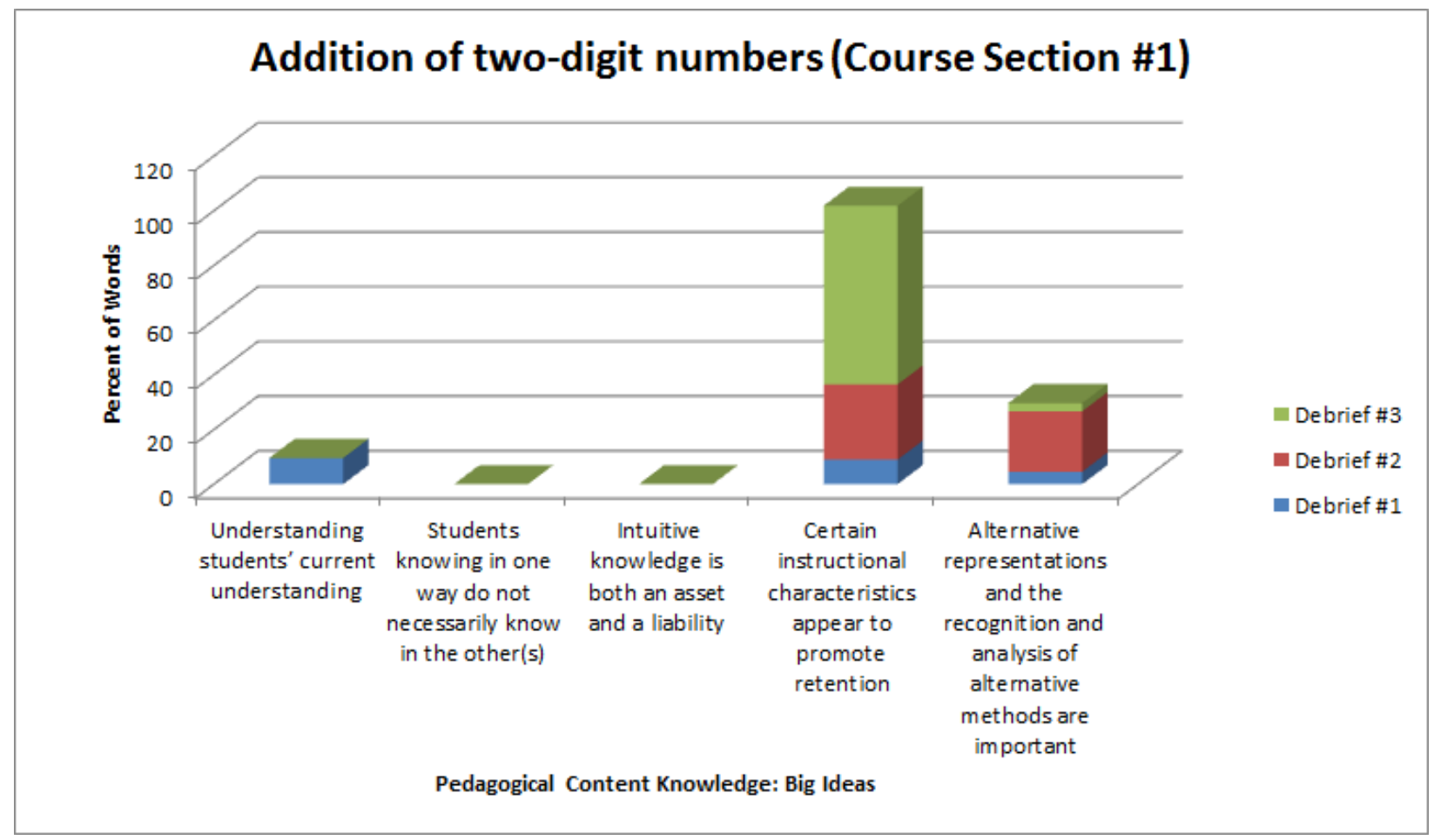

Figure 21. Bar graph of percent of words per Graeber's (1999) Big Ideas for Pedagogical Content Knowledge Discussions theme for Addition of two-digit numbers (Course Section \#3) group.

Similar to the first interaction structure, analysis of the discourse revealed few differences amongst the high and low selected groups. Again content knowledge discussions were minimal in comparison to discussions related to other themes, which supports the idea that growth in 
content knowledge may be a consequence of participants' actions independent of the debriefing sessions. Pedagogical content knowledge and lesson planning within the MLS structure discussions were again the focus of all the debriefing sessions, which supports the growth revealed by the analysis of the lesson plans from participants in this interaction structure. Also, similar to the findings from the first interaction structure, further analysis of pedagogical content knowledge discussions with respect to Graeber's (1999) big ideas for interaction structure two, revealed none of the groups discussed students knowing in one way do not necessarily know in the other(s) and intuitive knowledge is both an asset and a liability during any of the debriefing sessions. This supports the idea that the MLS mentor may also need to make an effort to address these aspects of pedagogical content knowledge during debriefing sessions as the analysis of the lesson plans revealed some growth for the second big idea and no growth for the third big idea. With respect to the MLS group issues theme, one of the low performing groups had the most discussion related to this topic during the first debriefing session followed minimal discussion in the second debriefing session and no discussing during the last debriefing session. This suggests the presence of the MLS mentor during the first debriefing session may have helped resolve the issues early in the cycle.

\section{Interaction Structure \#3}

For the third interaction structure, in which the MLS mentor participated in the last debriefing session only, the two groups with the highest total gains from the second preassessment to the post-assessment were Area Formulas (Course Section \#2) with a 37.5\% overall group gain and Area Formulas (Course Section \#3) with a 25\% overall group gain. The two groups with the least total gains from the second pre-assessment to the post-assessment were 
Comparing Fractions (Course Section \#1) with a -16.7\% overall group gain and Developing Equations (Course Section \#1) with a 0\% overall group gain.

\section{Groups with Highest Total Gains}

For the Area Formulas (Course Section \#2), with an overall group content gain of $37.5 \%$, the percent of total words for each theme for each of the debriefing sessions were calculated, see Table 26 and Figure 22. For each of the themes, the percent of totals words is given in order of debriefing session as follows: Content Knowledge Discussions, 30.414\%, 0\%, 32.166\%;

Pedagogical Content Knowledge Discussions, 43.779\%, 20.879\%, 20.683\%; MLS Group Issues, 0\%, 0\%, 0\%; Lesson Planning within MLS Structure, 25.806\%, 79.121\%, 47.154\%.

Interestingly, for the first debriefing session, the most discussions were related to pedagogical content knowledge followed by content knowledge, and then lesson planning within the MLS structure. This distribution changed for the second debriefing session, where lesson planning within the MLS structure became the most discussed theme followed by pedagogical content knowledge with no discussion of content knowledge. During the last debriefing session, in which the MLS mentor was present, the most discussed theme again was lesson planning within the MLS structure followed by content knowledge and then pedagogical content knowledge. No discussion occurred in the any of the debriefing sessions related to MLS group issues.

Table 26

Percentage of words per theme for debriefing sessions with Properties of Quadrilaterals (Course Section \#2) group

\begin{tabular}{|c|r|r|r|}
\hline Theme & Debrief \#1 & Debrief \#2 & Debrief \#3 \\
\hline Content Knowledge Discussions & 30.414 & 0 & 32.166 \\
\hline Pedagogical Content Knowledge Discussions & 43.779 & 20.879 & 20.683 \\
\hline MLS Group Issues & 0 & 0 & 0 \\
\hline Lesson Planning within MLS Structure & 25.806 & 79.121 & 47.154 \\
\hline
\end{tabular}




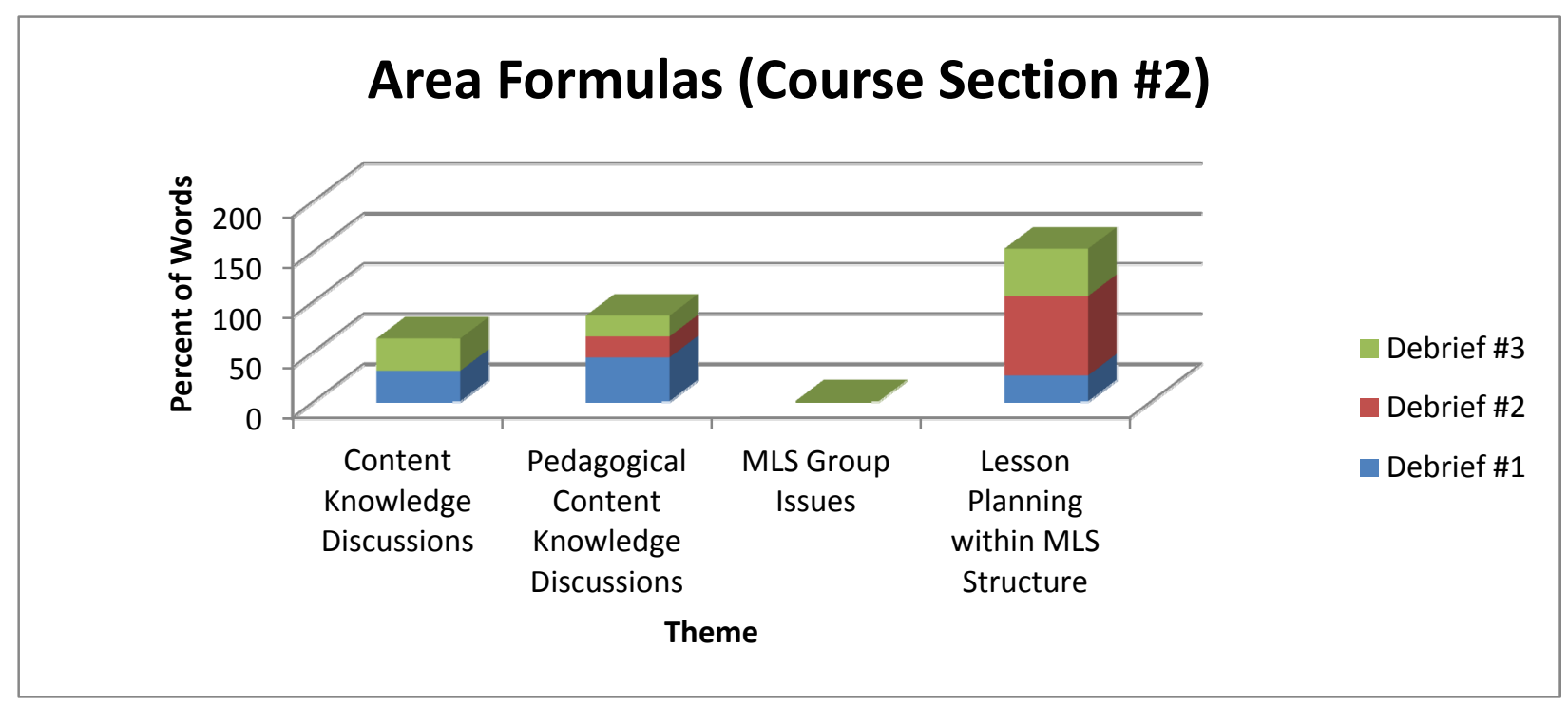

Figure 22. Bar graph of percent of words per theme for each debriefing session for Properties of Quadrilaterals (Course Section \#2) group.

The percent of total words for each of Graeber's (1999) big ideas within the Pedagogical Content Knowledge Discussions for each of the debriefing sessions were calculated for the Area Formulas (Course Section \#2) group, see Table 27 and Figure 23. It should be noted that the percents presented are out of the total percent of words for each debriefing session. For each of the big ideas, the percent of totals words is given in order of debriefing session as follows: Understanding students' current understanding, $14.286 \%, 0 \%, 8.918 \%$; Students knowing in one way do not necessarily know in the other(s), $0 \%, 0 \%, 0 \%$; Intuitive knowledge is both an asset and a liability, $0 \%, 0 \%, 0 \%$; Certain instructional characteristics appear to promote retention, $29.493 \%, 20.879 \%, 11.765 \%$; Alternative representations and the recognition and analysis of alternative methods are important, $0 \%, 0 \%, 0 \%$. This indicates all the discussions related to pedagogical content knowledge for the first and third debriefing sessions were related to understanding students' current understanding and certain instructional characteristics appear to promote retention. During the second debriefing session, only certain instructional characteristics appear to promote retention was discussed. No discussion was related students 
knowing in one way do not necessarily know in the other(s), intuitive knowledge is both an asset and a liability, or alternative representations and the recognition and analysis of alternative methods are important.

Table 27

Percentage of words per Graeber's (1999) Big Ideas for Pedagogical Content Knowledge Discussions theme with Area Formulas (Course Section \#2) group

\begin{tabular}{|l|l|l|l|}
\hline Pedagogical Content Knowledge: Big Ideas & Debrief \#1 & Debrief \#2 & Debrief \#3 \\
\hline Understanding students' current understanding & 14.286 & 0 & 8.918 \\
\hline Students knowing in one way do not necessarily know in the other(s) & 0 & 0 & 0 \\
\hline Intuitive knowledge is both an asset and a liability & 0 & 0 & 0 \\
\hline Certain instructional characteristics appear to promote retention & 29.493 & 20.879 & 11.765 \\
\hline $\begin{array}{l}\text { Alternative representations and the recognition and analysis of } \\
\text { alternative methods are important }\end{array}$ & 0 & 0 & \\
\hline
\end{tabular}

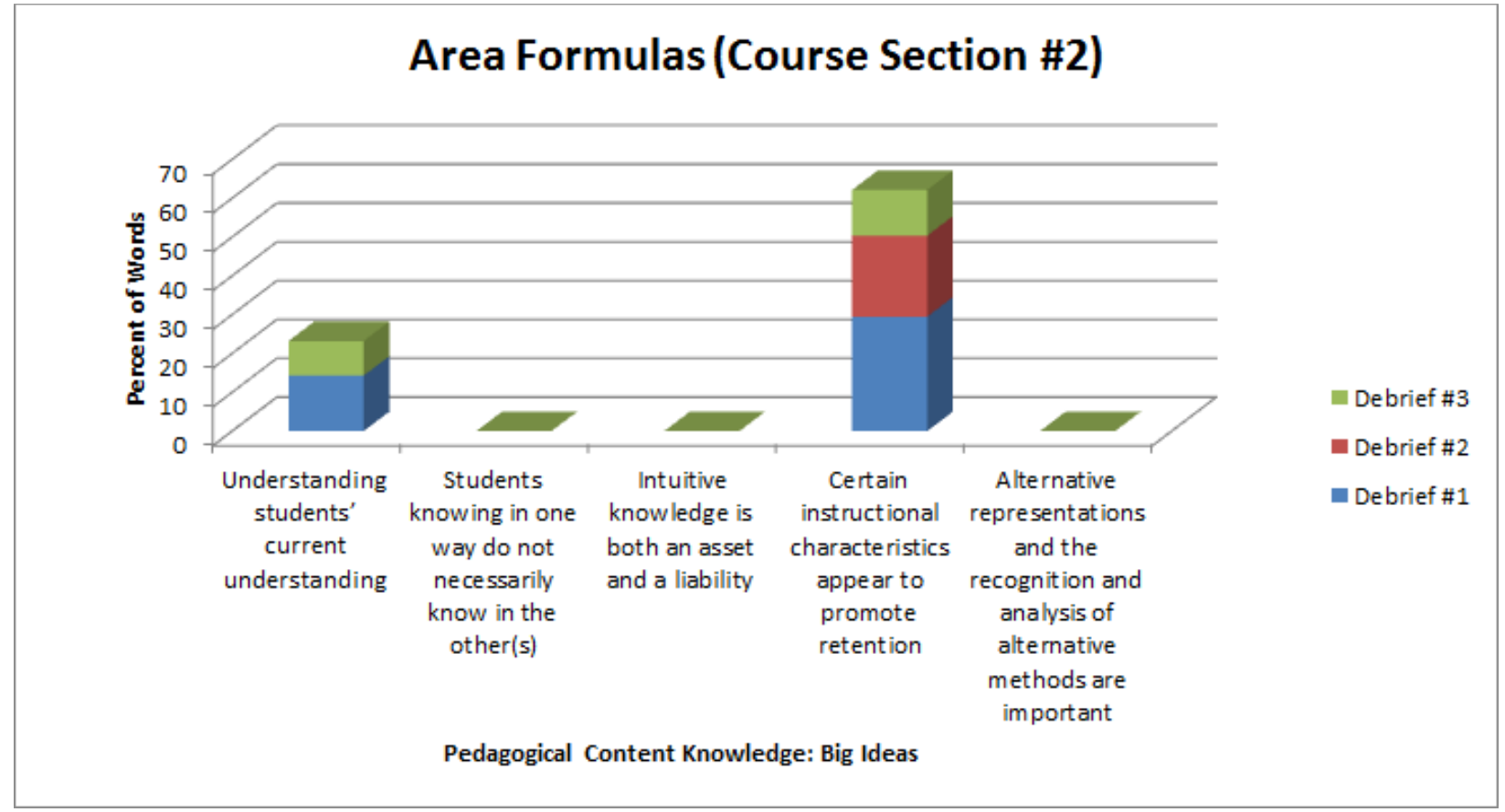

Figure 23. Bar graph of percent of words per Graeber's (1999) Big Idea for Pedagogical Content Knowledge Discussions theme for Area Formulas (Course Section \#2) group.

For the Area Formulas (Course Section \#3), with an overall group content gain of 25\%, the percent of total words for each theme for each of the debriefing sessions were calculated, see 
Table 28 and Figure 24. For each of the themes, the percent of totals words is given in order of debriefing session as follows: Content Knowledge Discussions, $0 \%, 0 \%, 0 \%$; Pedagogical Content Knowledge Discussions, 90.409\%, 33.548\%, 54.445\%; MLS Group Issues, 0\%, 66.451\%, 14.736\%; Lesson Planning within MLS Structure, 9.591\%, 0\%, 27.368\%. Similar to the other area formulas group, this group also spent the majority of the first debriefing session discussing pedagogical content knowledge with minor discussion of lesson planning within the MLS structure. However, during the second debriefing session, the group appears to have encountered some issues as most of the discussions related to MLS group issues with some discussion of pedagogical content knowledge. For the third debriefing session, which included the MLS mentor, the focus of the discussions was again pedagogical content knowledge followed by lesson planning within the MLS structure and MLS group issues. This may indicate that the MLS group issue was related to pedagogical content knowledge. Also, it is important note, content knowledge was not discussed during any of the debriefing sessions.

Table 28

Percentage of words per theme for debriefing sessions with Area Formulas (Course Section \#3) group

\begin{tabular}{|c|r|r|r|}
\hline Theme & Debrief \#1 & Debrief \#2 & Debrief \#3 \\
\hline Content Knowledge Discussions & 0 & 0 & 0 \\
\hline Pedagogical Content Knowledge Discussions & 90.409 & 33.548 & 54.445 \\
\hline MLS Group Issues & 0 & 66.451 & 14.736 \\
\hline Lesson Planning within MLS Structure & 9.591 & 0 & 27.368 \\
\hline
\end{tabular}




\section{Area Formulas (Course Section \#3)}

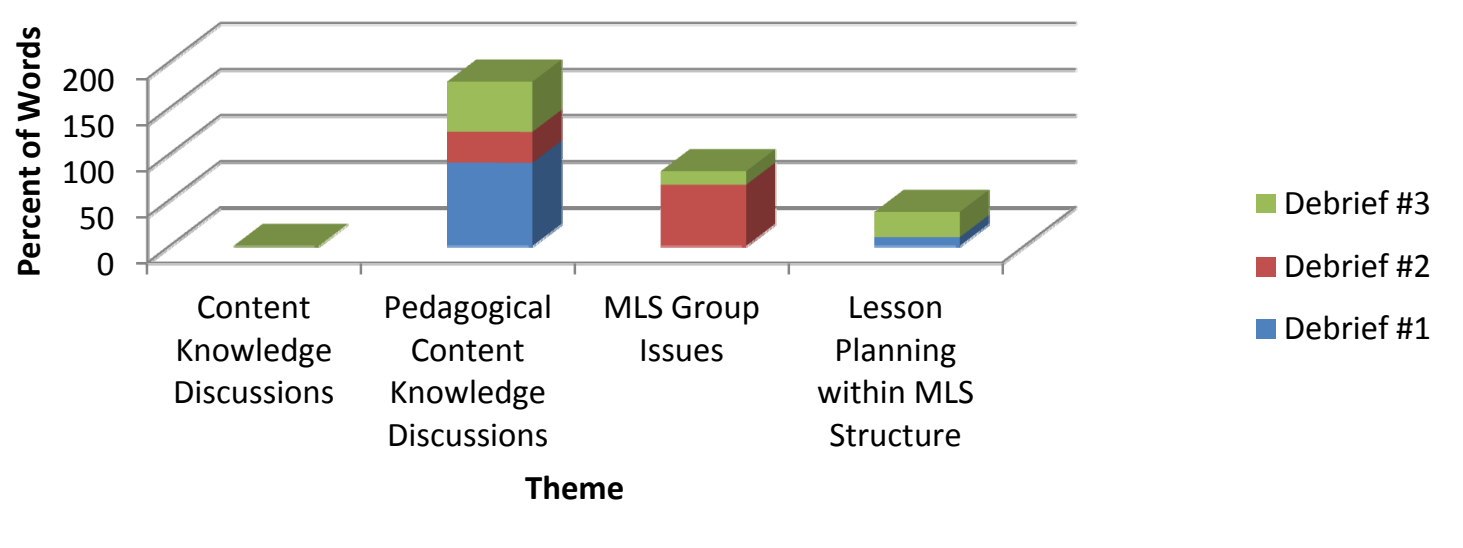

Figure 24. Bar graph of percent of words per theme for each debriefing session for Area Formulas (Course Section \#3) group.

The percent of total words for each of Graeber's (1999) big ideas within the Pedagogical Content Knowledge Discussions for each of the debriefing sessions were calculated for the Properties of Quadrilaterals (Course Section \#1) group, see Table 29 and Figure 25. For each of the big ideas, the percent of totals words is given in order of debriefing session as follows: Understanding students' current understanding, 6.419\%, 42.103\%, 22.222\%; Students knowing in one way do not necessarily know in the other(s), $0 \%, 0 \%, 0 \%$; Intuitive knowledge is both an asset and a liability, $0 \%, 0 \%, 0 \%$; Certain instructional characteristics appear to promote retention, $14.039 \%, 0 \%, 43.537 \%$; Alternative representations and the recognition and analysis of alternative methods are important, $0 \%, 28.77 \%, 0 \%$. For this group, most of the discussions related to Pedagogical Content Knowledge for the first and third debriefing sessions were related to certain instructional characteristics appear to promote retention, while the focus of the second debriefing session discussions was understanding students' current understanding. Also, notable is the remaining discussions related to Pedagogical Content Knowledge for the first and third debriefing sessions was understanding students' current understanding, but for the second debriefing session the remaining discussion was related to alternative representations and the 
recognition and analysis of alternative methods are important. No discussions, for any of the debriefing sessions, involved students knowing in one way do not necessarily know in the other(s) or intuitive knowledge is both an asset and a liability.

Table 29

Percentage of words per Graeber's (1999) Big Ideas for Pedagogical Content Knowledge Discussions theme for Area Formulas (Course Section \#3) group

\begin{tabular}{|c|r|r|r|}
\hline Pedagogical Content Knowledge: Big Ideas & Debrief \#1 & Debrief \#2 & Debrief \#3 \\
\hline $\begin{array}{c}\text { Understanding students' current understanding } \\
\text { other(s) }\end{array}$ & 6.419 & 42.103 & 22.222 \\
\hline $\begin{array}{c}\text { Students knowing in one way do not necessarily know in the } \\
\text { retention }\end{array}$ & 0 & 0 & 0 \\
\hline $\begin{array}{c}\text { Certain instructional characteristics appear to promote } \\
\text { alternative representations and the recognition and analysis of } \\
\text { alternative methods are important }\end{array}$ & 14.039 & 0 & 43.537 \\
\hline
\end{tabular}

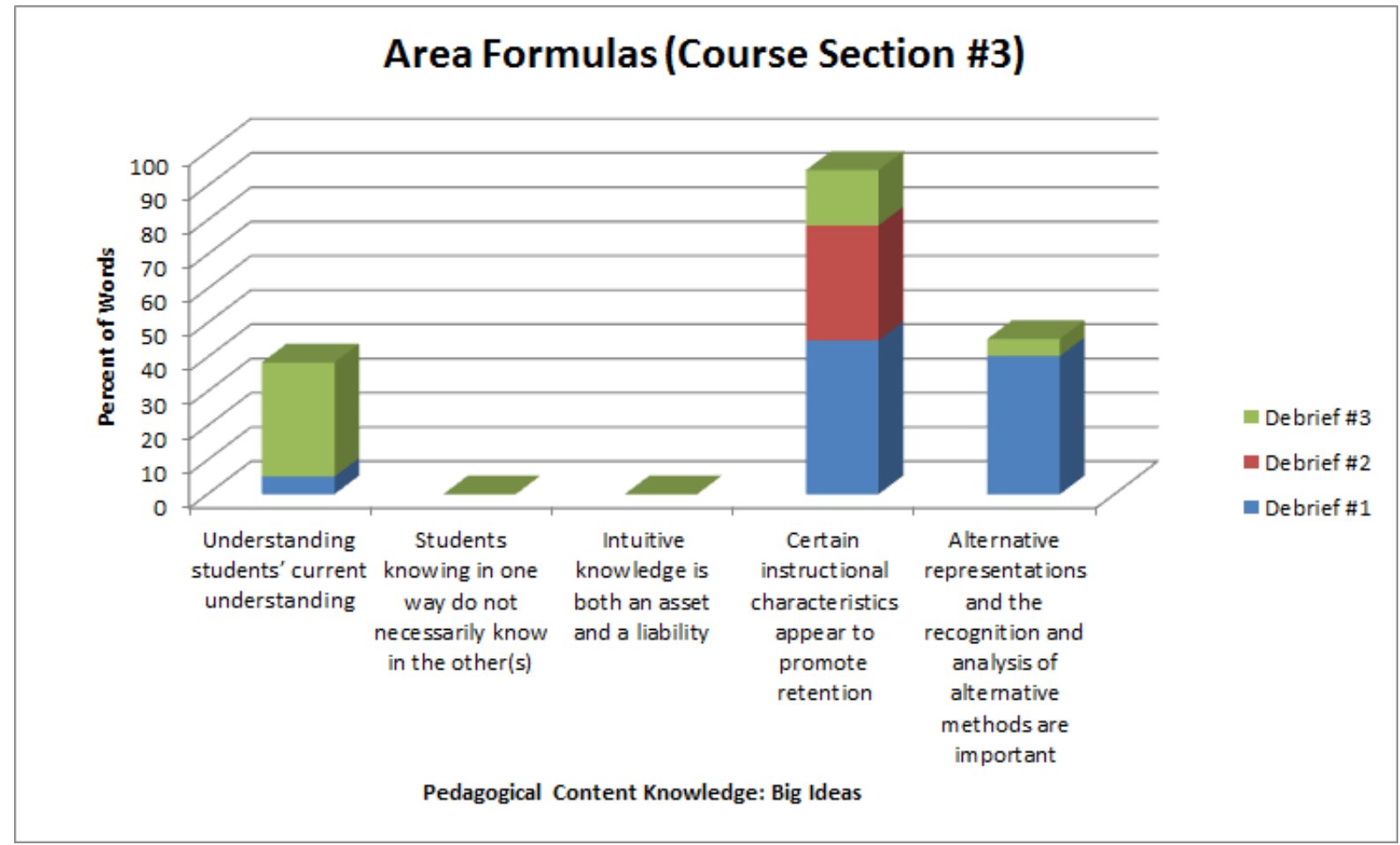

Figure 25. Bar graph of percent of words per Graeber's (1999) Big Ideas for Pedagogical Content Knowledge Discussions theme for Area Formulas (Course Section \#3) group. 
For the Comparing Fractions (Course Section \#1), with an overall group content gain of $16.7 \%$, the percent of total words for each theme for each of the debriefing sessions were calculated, see Table 30 and Figure 26. For each of the themes, the percent of totals words is given in order of debriefing session as follows: Content Knowledge Discussions, $0 \%, 0 \%$, 4.911\%; Pedagogical Content Knowledge Discussions, 64.677\%, 92.486\%, 41.647\%; MLS Group Issues, 0\%, 0\%, 27.395\%; Lesson Planning within MLS Structure, 35.323\%, 7.514\%, $26.047 \%$. Similar to the two highest overall content gains' groups, pedagogical content knowledge discussions were the focus of the debriefing sessions. For the comparing fractions group, this was the case for all three debriefing sessions. Interestingly, although during the first two debriefing sessions without the MLS mentor, the group had no discussions related to MLS group issues, during the third debriefing session, the theme was discussed. This may indicate that in the absence of the MLS mentor, the group did not address issues that were needed for the group to progress in their lesson development. Also, important to note is lesson planning within the MLS structure was discussed in all three debriefing sessions, while content knowledge was discussed minimally during the last debriefing session. The lack of content knowledge discussion may explain the lack of growth in this area for this group.

Table 30

Percentage of words per theme for debriefing sessions with Comparing Fractions (Course Section \#1) group

\begin{tabular}{|c|l|l|l|}
\hline Theme & Debrief \#1 & Debrief \#2 & Debrief \#3 \\
\hline Content Knowledge Discussions & 0 & 0 & 4.911 \\
\hline Pedagogical Content Knowledge Discussions & 64.677 & 92.486 & 41.647 \\
\hline MLS Group Issues & 0 & 0 & 27.395 \\
\hline Lesson Planning within MLS Structure & 35.323 & 7.514 & 26.047 \\
\hline
\end{tabular}




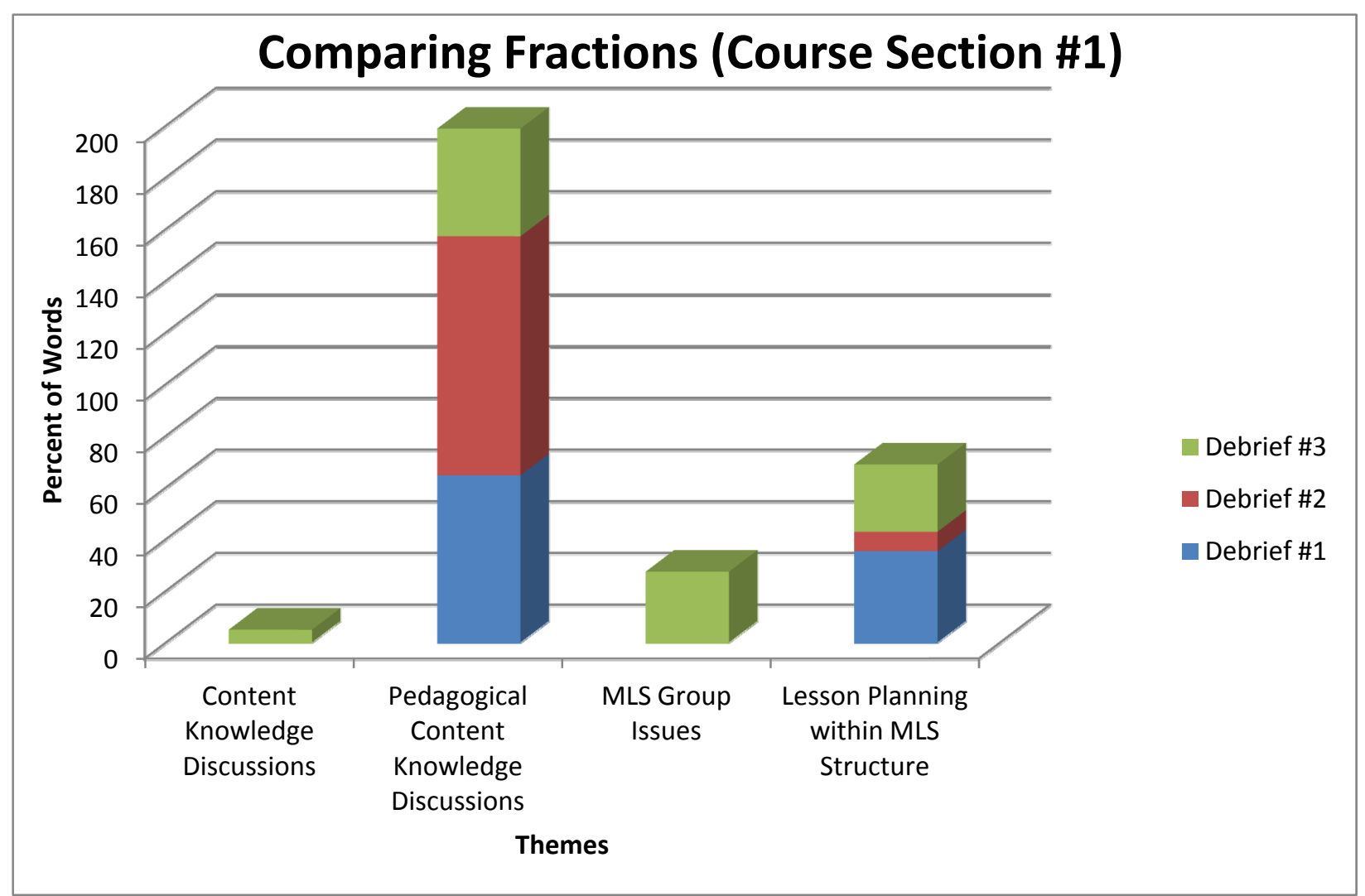

Figure 26. Bar graph of percent of words per theme for each debriefing session for Adding Fractions (Course Section \#1) group.

The percent of total words for each of Graeber's (1999) big ideas within the Pedagogical Content Knowledge Discussions for each of the debriefing sessions were calculated for the Comparing Fractions (Course Section \#3) group, see Table 31 and Figure 27. For each of the big ideas, the percent of totals words is given in order of debriefing session as follows:

Understanding students' current understanding, 0\%, 28.902\%, 13.072\%; Students knowing in one way do not necessarily know in the other(s), $0 \%, 0 \%, 0 \%$; Intuitive knowledge is both an asset and a liability, $0 \%, 0 \%, 0 \%$; Certain instructional characteristics appear to promote retention, $64.677 \%, 63.584 \%, 18.127 \%$; Alternative representations and the recognition and analysis of alternative methods are important, $0 \%, 0 \%, 10.448 \%$. For this group, most of the discussions related to pedagogical content knowledge for all the debriefing sessions were related to certain instructional characteristics appear to promote retention. Also, notable is for the 
second and third debriefing sessions, understanding students' current understanding, was the only one of Graeber's (1999) five big ideas discussed. During the third debriefing session, some discussion was related to alternative representations and the recognition and analysis of alternative methods are important. No discussions, for any of the debriefing sessions, involved students knowing in one way do not necessarily know in the other(s) or intuitive knowledge is both an asset and a liability.

Table 31

Percentage of words per Graeber's (1999) Big Ideas for Pedagogical Content Knowledge Discussions theme for Adding Fractions (Course Section \#1) group

\begin{tabular}{|c|r|r|r|}
\hline $\begin{array}{c}\text { Pedagogical Content Knowledge: Big Ideas } \\
\text { Understanding students' current understanding }\end{array}$ & $\begin{array}{l}\text { Debrief } \\
\# 1\end{array}$ & $\begin{array}{l}\text { Debrief } \\
\# 2\end{array}$ & \multicolumn{1}{l|}{$\begin{array}{l}\text { Debrief } \\
\# 3\end{array}$} \\
\hline $\begin{array}{c}\text { Students knowing in one way do not necessarily know in the } \\
\text { other(s) }\end{array}$ & 0 & 28.902 & 13.072 \\
\hline $\begin{array}{c}\text { Intuitive knowledge is both an asset and a liability } \\
\text { Certain instructional characteristics appear to promote retention }\end{array}$ & 0 & 0 & 0 \\
\hline $\begin{array}{c}\text { Alternative representations and the recognition and analysis of } \\
\text { alternative methods are important }\end{array}$ & 0 & 0 & 0 \\
\hline
\end{tabular}

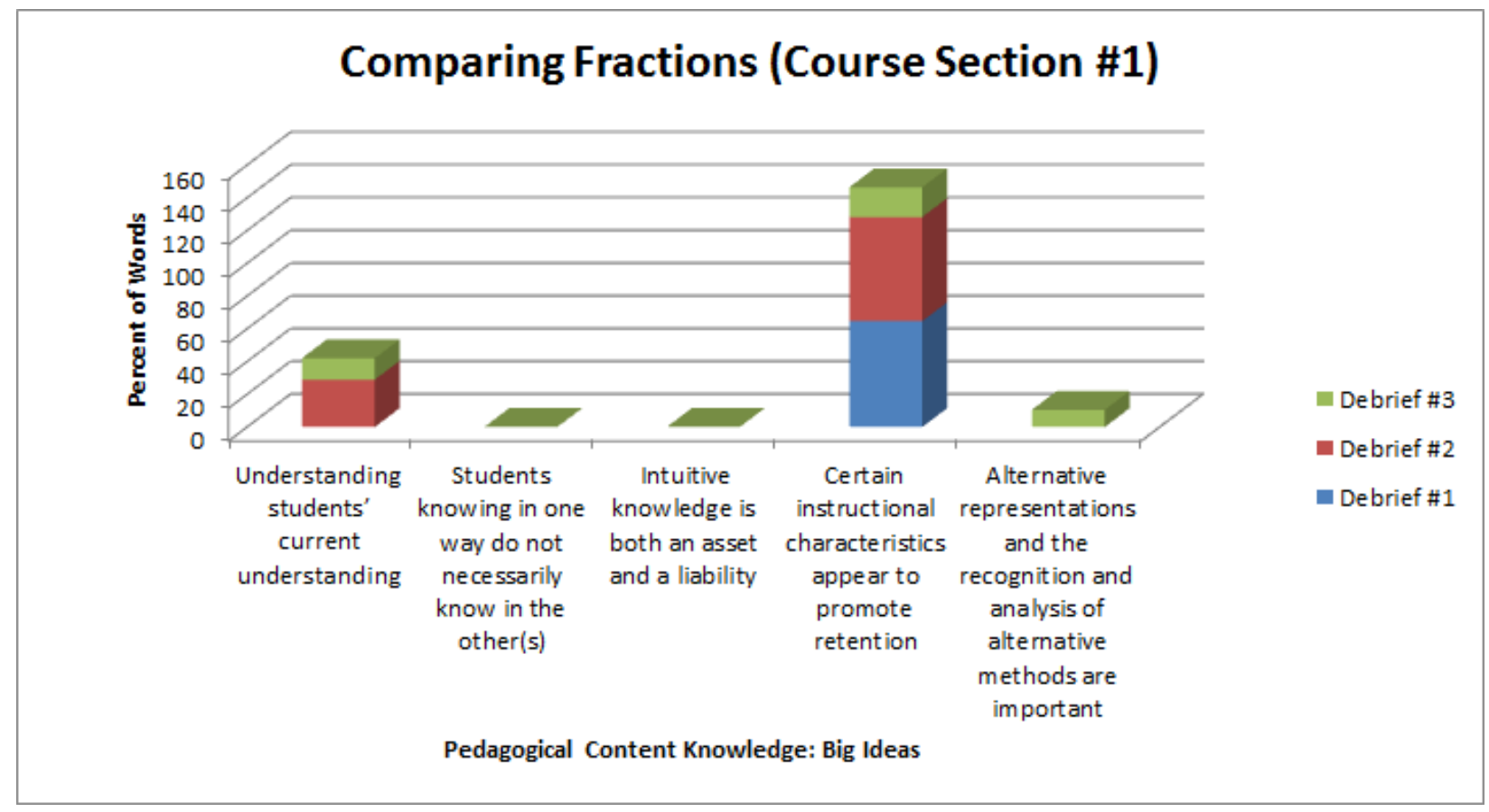

Figure 27. Bar graph of percent of words per Graeber's (1999) Big Ideas for Pedagogical Content Knowledge Discussions theme for Comparing Fractions (Course Section \#1) group. 


\section{Groups with Least Total Gains}

For the Developing Equations group (Course Section \#1), with an overall group content gain of $0 \%$, the percent of total words for each theme for each of the debriefing sessions were calculated, see Table 32 and Figure 28. For each of the themes, the percent of totals words is given in order of debriefing session as follows: Content Knowledge Discussions, $0 \%, 0 \%, 0 \%$; Pedagogical Content Knowledge Discussions, 39.942\%, 60.714\%, 57.124\%; MLS Group Issues, 0\%, 0\%, 1.943\%; Lesson Planning within MLS Structure, 60.058\%, 39.286\%, 40.929\%. For this group, the most discussed theme for the first debriefing session was lesson planning within the MLS structure. For the other two debriefing sessions, the most discussed theme was pedagogical content knowledge followed by lesson planning within the MLS structue. No discussion during any of the debriefing sessions related to content knowledge, which could explain the lack of growth in content knowledge for this group. Also, it should be noted that during the third debriefing session, some minimal discussion related to MLS group issues took place.

Table 32

Percentage of words per theme for debriefing sessions for Developing Equations (Course Section \#1) group

\begin{tabular}{|c|r|r|r|}
\hline \multicolumn{1}{|l|}{ Theme } & \multicolumn{1}{c|}{ Debrief \#1 } & \multicolumn{1}{l|}{ Debrief \#2 } & \multicolumn{1}{l|}{ Debrief \#3 } \\
\hline Content Knowledge Discussions & 0 & 0 & 0 \\
\hline Pedagogical Content Knowledge Discussions & 39.942 & 60.714 & 57.124 \\
\hline MLS Group Issues & 0 & 0 & 1.943 \\
\hline Lesson Planning within MLS Structure & 60.058 & 39.286 & 40.929 \\
\hline
\end{tabular}




\section{Developing Equations (Course Section \#1)}

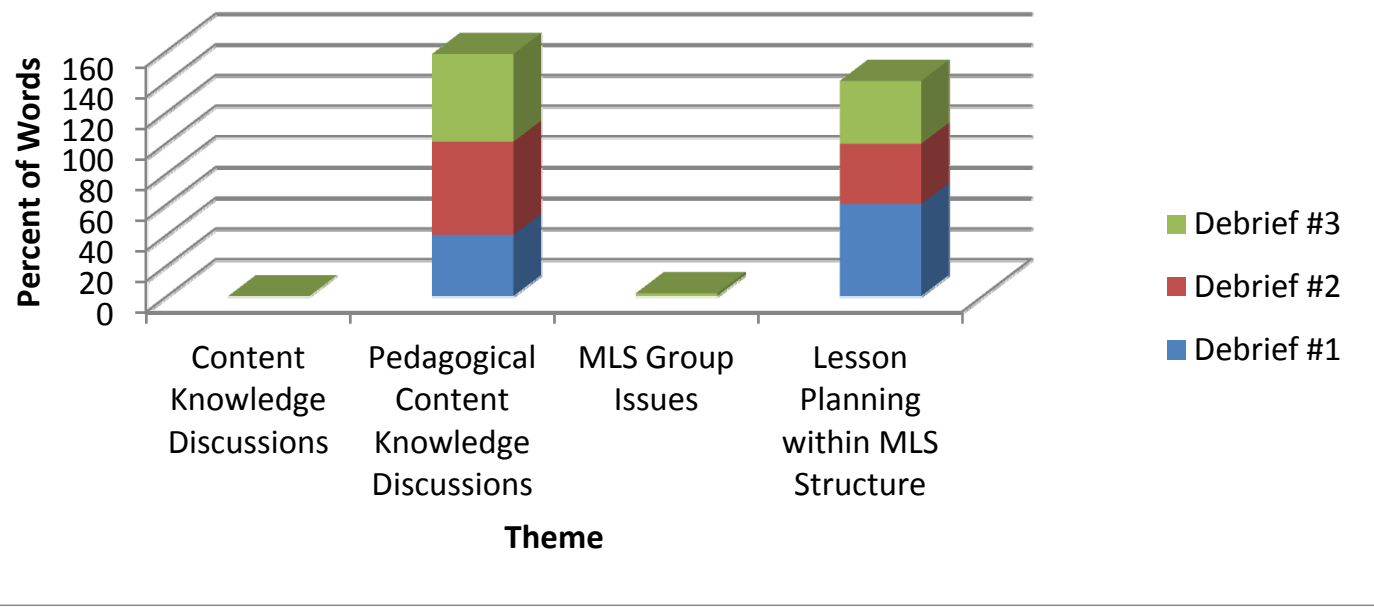

Figure 28. Bar graph of percent of words per theme for each debriefing session for Developing Equations (Course Section \#1) group.

The percent of total words for each of Graeber's (1999) big ideas within the Pedagogical Content Knowledge Discussions for each of the debriefing sessions were calculated for the Developing Equations group (Course Section \#1), see Table 33 and Figure 29. For each of the big ideas, the percent of totals words is given in order of debriefing session as follows: Understanding students' current understanding, 0\%, 27.381\%, 18.021\%; Students knowing in one way do not necessarily know in the other(s), 0\%, 0\%, 0\%; Intuitive knowledge is both an asset and a liability, $0 \%, 0 \%, 0 \%$; Certain instructional characteristics appear to promote retention, 39.942\%, 33.333\%, 25.852\%; Alternative representations and the recognition and analysis of alternative methods are important, $0 \%, 0 \%, 13.251 \%$. For this group, most of the pedagogical content knowledge discussions for all the debriefing sessions were related to certain instructional characteristics appear to promote retention. For the second and third debriefing sessions, understanding students' current understanding, was also discussed heavily. Minimal discussion related to alternative representations and the recognition and analysis of alternative methods are important took place in the third debriefing session only. No discussions, for any of 
the debriefing sessions, involved students knowing in one way do not necessarily know in the other(s) or intuitive knowledge is both an asset and a liability.

Table 33

Percentage of words per Graeber's (1999) Big Ideas for Pedagogical Content Knowledge Discussions theme for Developing Equations (Course Section \#1) group

\begin{tabular}{|c|c|c|c|}
\hline Pedagogical Content Knowledge: Big Ideas & $\begin{array}{l}\text { Debrief } \\
\# 1\end{array}$ & $\begin{array}{l}\text { Debrief } \\
\# 2\end{array}$ & $\begin{array}{l}\text { Debrief } \\
\# 3\end{array}$ \\
\hline Understanding students' current understanding & 0 & 27.381 & 18.021 \\
\hline $\begin{array}{l}\text { Students knowing in one way do not necessarily know in the } \\
\text { other(s) }\end{array}$ & 0 & 0 & 0 \\
\hline Intuitive knowledge is both an asset and a liability & 0 & 0 & 0 \\
\hline $\begin{array}{l}\text { Certain instructional characteristics appear to promote } \\
\text { retention }\end{array}$ & 39.942 & 33.333 & 25.852 \\
\hline $\begin{array}{c}\text { Alternative representations and the recognition and analysis of } \\
\text { alternative methods are important }\end{array}$ & 0 & 0 & 13.251 \\
\hline
\end{tabular}

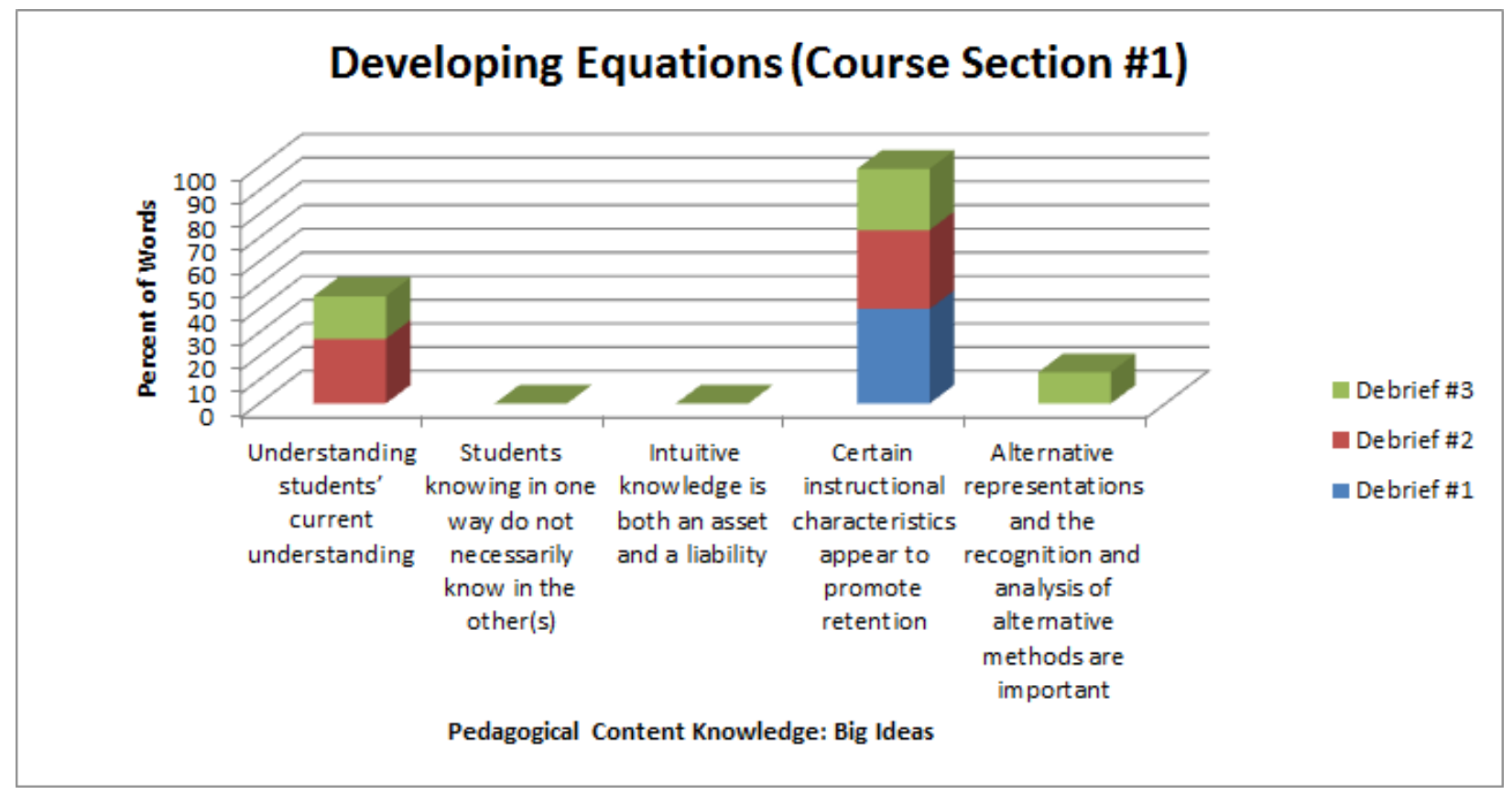

Figure 29. Bar graph of percent of words per Graeber's (1999) Big Ideas for Pedagogical Content Knowledge Discussions theme for Developing Equations (Course Section \#1) group.

Results of the analysis of the four selected groups for the third interaction structure were similar to those from the first and second interaction structure. Again, content knowledge was discussed minimally with the exception of the highest performing content knowledge group. 
Also, similar to the findings from the first and second interaction structure, pedagogical content knowledge and lesson planning within the MLS structure were both heavily discussed themes. However, in contrast to the findings from the first and second interaction structures, the results from the analysis of the lesson plans did not reveal much growth in pedagogical content knowledge for two of the big ideas and no growth at all for three of the big ideas. This suggests the quality of the discussions during the debriefing sessions without the MLS mentor may not have been similar to the discussions in which the MLS mentor was present.

As with the first and second interaction structure analysis, further analysis of the discourse with respect to Graeber's (1999) big ideas for pedagogical content knowledge, revealed no group had any discussion during any of the debriefing sessions related to students knowing in one way do not necessarily know in the other(s) or intuitive knowledge is both an asset and a liability. However, for all four selected groups, certain instructional characteristics appear to promote retention, was the most heavily discussed big idea followed by understanding students' current understanding. Minimal discussion related to alternative representations and the recognition and analysis of alternative methods are important. Two of the four groups had no discussions related to MLS group issues, while the other two group had discussions beginning in the second and third debriefing sessions, respectively. This could indicate the need for the MLS mentor to participate in the first debriefing sessions, so that a delay in addressing these issues does not occur.

\section{Feedback Survey}

Average responses to the Likert-type items on the feedback survey were calculated per interaction structure and as a whole group, see Table 34. As discussed previously, statements were rated on a 1 to 5 scale, with a score of 5 indicating strongly agree and a score of 1 
indicating strongly disagree. Some items were phrased in the negative form of the statement. After analysis, items were grouped according to statements related to assigned interaction structure and themes from the discourse analysis, which included content knowledge, pedagogical content knowledge, lesson planning, and MLS related questions.

A total of three items on the feedback survey were intended to capture participants' perception of how their assigned interaction structure affected various pieces of the MLS experience. For the first item, "Input from the MLS Mentor (i.e., instructor) was helpful as we designed our lesson," average responses for each interaction structure were 4.65, 4.75, and 3.71 and standard deviations of $0.49,0.44$, and 1.51 , respectively with a complete group average of 4.31 and a standard deviation of 1.11. For the second item, "Input from the MLS Mentor (ie., instructor) was helpful in understanding the content of our lesson," average responses for each interaction structure were $4.04,3.8$, and 3.5 and standard deviations of $1.11,1.54$, and 1.32 , respectively with a complete group average of 3.76 and a standard deviation of 1.32 . For the third item, "I would have liked to have more input or feedback on our group lesson from the MLS Mentor (ie., instructor) during the teaching and analysis phases of the project," average responses for each interaction structure were $2.78,3.3$, and 3.7 and standard deviations of 1.09, 1.34 , and 1.12 , respectively with a complete group average of 3.30 and a standard deviation of 1.22. Overall, these results suggest participants assigned to interaction structure three would have liked more feedback from the MLS mentor when compared with participants assigned to the first two interaction structures. Given that for interaction structure three, the MLS mentor only participated in the last debriefing session, this result indicates participants valued the presence of the MLS mentor in the earlier debriefing sessions. 
A total of three items on the feedback survey were intended to capture participants' perception of how their assigned interaction structure affected development of their content knowledge during the MLS experience. For the first item, "Planning together with other group members helped me deepen my knowledge of the mathematics topic we taught," average responses for each interaction structure were 4.09, 3.9, and 4.04 and standard deviations of 1.04, 0.79 , and 0.96 , respectively with a complete group average of 4.01 and a standard deviation of 0.93. For the second item, "The actual teaching of the lesson helped me deepen my understanding of the mathematics topic," average responses for each interaction structure were $4.17,4.3$, and 4.32 and standard deviations of $0.72,1.13$, and 0.90 , respectively with a complete group average of 4.27 and a standard deviation of 0.91 . For the third item, "Preparing to teach this topic and planning the lesson caused me to engage in mathematical reasoning and problem solving," average responses for each interaction structure were 4.17, 4.3, and 4.29 and standard deviations of $0.83,0.66$, and 0.66 , respectively with a complete group average of 4.29 and a standard deviation of 0.71 . For each interaction structure, average responses to all items related to content knowledge were high and differences in averages were minimal. This suggests participants, irrespective of assigned interaction structure, felt their content knowledge had developed as a consequence of their participation in MLS.

One item on the feedback survey was intended to capture participants' perception of how their assigned interaction structure affected development of their pedagogical content knowledge during the MLS experience. For the item, "MLS deepened my ability to anticipate student responses to mathematical questions and tasks," average responses for each interaction structure were $4.11,3.95$, and 4.11 and standard deviations of $0.93,0.76$, and 0.88 , respectively with a complete group average of 4.06 and a standard deviation of 0.85 . For the first and third 
interaction structure, average responses to all items related to pedagogical content knowledge were the same and high with the average response from participants in the second interaction structure only slightly less. This suggests participants, irrespective of assigned interaction structure, felt their pedagogical content knowledge had developed as a consequence of their participation in MLS, which supports the findings from the pedagogical content analysis portion of this study.

A total of four items on the feedback survey were intended to capture participants' perception of how their assigned interaction structure affected development of their lesson planning ability during the MLS experience. For the first item, "Planning together with other group members helped me broaden my knowledge of possible ways of teaching the desired lesson," average responses for each interaction structure were 4.35, 4.25, and 4.14 and standard deviations of $1.03,0.79$, and 1.01 , respectively with a complete group average of 4.24 and a standard deviation of 0.95 . For the second item, "Analyzing each others teaching of the lesson helped me think more deeply about my own teaching," average responses for each interaction structure were $4.61,4.65$, and 4.54 and standard deviations of $0.66,0.49$, and 0.58 , respectively with a complete group average of 4.59 and a standard deviation of 0.57 . For the third item, "Feedback from my group members helped me understand my teaching strengths and areas for improvement," average responses for each interaction structure were 4.35, 4.3, and 4.36 and standard deviations of $0.93,0.57$, and 0.91 , respectively with a complete group average of 4.34 and a standard deviation of 0.83 . For the fourth item, "Teaching strategies I learned through MLS will be useful when I teach elementary school students," average responses for each interaction structure were $4.43,4.7$, and 4.46 and standard deviations of $0.73,0.47$, and 0.74 , respectively with a complete group average of 4.52 and a standard deviation of 0.67 . For each 
interaction structure, average responses to all items related to lesson planning were high and differences in averages were minimal. For the fourth item, the average response from the second interaction structure was slightly higher when compared to the first and third interaction structure average responses, but not significantly higher. Overall, the results indicate all participants regardless of assigned interaction structure felt their lesson planning had developed as a consequence of their participation in MLS.

Two items on the feedback survey were intended to capture participants' perception of the MLS experience in general. For the first item, "When I analyzed my group members' teaching of the lesson, my concern for their feelings influenced my assessment and feedback," average responses for each interaction structure were $2.74,2.68$, and 3.18 and standard deviations of $1.25,1.10$, and 1.22 , respectively with a complete group average of 2.89 with a standard deviation of 1.20. These averages would correspond to a neutral rating by the participants indicating the participants were not overly concerned with their group members' feelings when providing feedback. For the second item, "I would not like to engage in MLS in other contexts (ie. other courses or as a practicing teacher)" average responses for each interaction structure were 1.83, 2.3, and 2.14 and standard deviations of $0.72,0.98$, and 1.04, respectively with a complete group average of 2.08 with a standard deviation of 0.94 . This suggests participants in all assigned interaction structures valued the MLS experience and would have liked to have engaged in similar experiences in other courses. 
Table 34

Average Response and Standard Deviatons to Likert-type Statements on Feedback Survey

\begin{tabular}{|c|c|c|c|c|c|}
\hline & Item & $\begin{array}{c}\text { Int Str 1 } \\
\text { Average \& } \\
\text { Standard } \\
\text { Deviation }\end{array}$ & $\begin{array}{c}\text { Int Str 2 } \\
\text { Average \& } \\
\text { Standard } \\
\text { Deviation }\end{array}$ & $\begin{array}{c}\text { Int Str 3 } \\
\text { Average \& } \\
\text { Standard } \\
\text { Deviation }\end{array}$ & $\begin{array}{l}\text { All Part } \\
\text { Average \& } \\
\text { Standard } \\
\text { Deviation }\end{array}$ \\
\hline \multirow{3}{*}{ 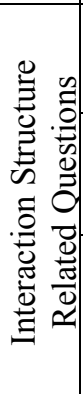 } & $\begin{array}{l}\text { Input from the MLS Mentor (ie., instructor) was } \\
\text { helpful as we designed our lesson. }\end{array}$ & $\begin{array}{l}4.65 \\
0.49^{*}\end{array}$ & $\begin{array}{l}4.75 \\
0.44\end{array}$ & $\begin{array}{l}3.71 \\
1.51\end{array}$ & $\begin{array}{l}4.31 \\
1.11\end{array}$ \\
\hline & $\begin{array}{l}\text { Input from the MLS Mentor (ie., instructor) was } \\
\text { helpful in understanding the content of our } \\
\text { lesson. }\end{array}$ & $\begin{array}{l}4.04 \\
1.11\end{array}$ & $\begin{array}{l}3.8 \\
1.54\end{array}$ & $\begin{array}{l}3.5 \\
1.32\end{array}$ & $\begin{array}{l}3.76 \\
1.32\end{array}$ \\
\hline & $\begin{array}{l}\text { I would have liked to have more input or } \\
\text { feedback on our group lesson from the MLS } \\
\text { Mentor (ie., instructor) during the teaching and } \\
\text { analysis phases of the project. }\end{array}$ & $\begin{array}{l}2.78 \\
1.09\end{array}$ & $\begin{array}{l}3.3 \\
1.34\end{array}$ & $\begin{array}{l}3.7 \\
1.12\end{array}$ & $\begin{array}{l}3.30 \\
1.22\end{array}$ \\
\hline \multirow{3}{*}{ 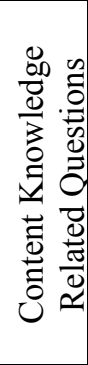 } & $\begin{array}{l}\text { Planning together with other group members } \\
\text { helped me deepen my knowledge of the } \\
\text { mathematics topic we taught. }\end{array}$ & $\begin{array}{l}4.09 \\
1.04\end{array}$ & $\begin{array}{l}3.9 \\
0.79\end{array}$ & $\begin{array}{l}4.04 \\
0.96\end{array}$ & $\begin{array}{l}4.01 \\
0.93\end{array}$ \\
\hline & $\begin{array}{l}\text { The actual teaching of the lesson helped me } \\
\text { deepen my understanding of the mathematics } \\
\text { topic. }\end{array}$ & $\begin{array}{l}4.17 \\
0.72\end{array}$ & $\begin{array}{l}4.3 \\
1.13\end{array}$ & $\begin{array}{l}4.32 \\
0.90\end{array}$ & $\begin{array}{l}4.27 \\
0.91\end{array}$ \\
\hline & $\begin{array}{l}\text { Preparing to teach this topic and planning the } \\
\text { lesson caused me to engage in mathematical } \\
\text { reasoning and problem solving. }\end{array}$ & $\begin{array}{l}4.17 \\
0.83\end{array}$ & $\begin{array}{l}4.3 \\
0.66\end{array}$ & $\begin{array}{l}4.29 \\
0.66\end{array}$ & $\begin{array}{l}4.25 \\
0.71\end{array}$ \\
\hline 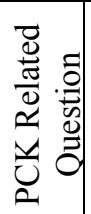 & $\begin{array}{l}\text { MLS deepened my ability to anticipate student } \\
\text { responses to mathematical questions and tasks. }\end{array}$ & $\begin{array}{l}4.11 \\
0.93\end{array}$ & $\begin{array}{c}3.95 \\
0.76\end{array}$ & $\begin{array}{l}4.11 \\
0.88\end{array}$ & $\begin{array}{l}4.06 \\
0.85\end{array}$ \\
\hline \multirow{4}{*}{ 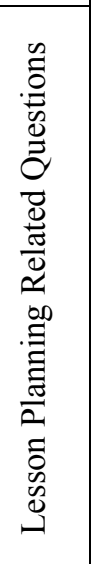 } & $\begin{array}{l}\text { Planning together with other group members } \\
\text { helped me broaden my knowledge of possible } \\
\text { ways of teaching the desired lesson. }\end{array}$ & $\begin{array}{l}4.35 \\
1.03\end{array}$ & $\begin{array}{l}4.25 \\
0.79\end{array}$ & $\begin{array}{l}4.14 \\
1.01\end{array}$ & $\begin{array}{l}4.24 \\
0.95\end{array}$ \\
\hline & $\begin{array}{l}\text { Analyzing each others teaching of the lesson } \\
\text { helped me think more deeply about my own } \\
\text { teaching. }\end{array}$ & $\begin{array}{l}4.61 \\
0.66\end{array}$ & $\begin{array}{l}4.65 \\
0.49\end{array}$ & $\begin{array}{l}4.54 \\
0.58\end{array}$ & $\begin{array}{l}4.59 \\
0.57\end{array}$ \\
\hline & $\begin{array}{l}\text { Feedback from my group members helped me } \\
\text { understand my teaching strengths and areas for } \\
\text { improvement. }\end{array}$ & $\begin{array}{c}4.35 \\
0.93\end{array}$ & $\begin{array}{l}4.3, \\
0.57\end{array}$ & $\begin{array}{c}4.36, \\
0.91\end{array}$ & $\begin{array}{l}4.34, \\
0.83\end{array}$ \\
\hline & $\begin{array}{l}\text { Teaching strategies I learned through MLS will } \\
\text { be useful when I teach elementary school } \\
\text { students. }\end{array}$ & $\begin{array}{l}4.43 \\
0.73\end{array}$ & $\begin{array}{l}4.7 \\
0.47\end{array}$ & $\begin{array}{c}4.46, \\
0.74\end{array}$ & $\begin{array}{l}4.52, \\
0.67\end{array}$ \\
\hline \multirow{2}{*}{ 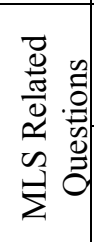 } & $\begin{array}{l}\text { When I analyzed my group members' teaching } \\
\text { of the lesson, my concern for their feelings } \\
\text { influenced my assessment and feedback. }\end{array}$ & $\begin{array}{c}2.74 \\
1.25\end{array}$ & $\begin{array}{c}2.68 \\
1.10\end{array}$ & $\begin{array}{c}3.18, \\
1.22\end{array}$ & $\begin{array}{c}2.89 \\
1.20\end{array}$ \\
\hline & $\begin{array}{l}\text { I would not like to engage in MLS in other } \\
\text { contexts (ie. other courses or as a practicing } \\
\text { teacher). }\end{array}$ & $\begin{array}{l}1.83 \\
0.72\end{array}$ & $\begin{array}{l}2.3, \\
0.98\end{array}$ & $\begin{array}{c}2.14, \\
1.04\end{array}$ & $\begin{array}{c}2.08, \\
0.94\end{array}$ \\
\hline
\end{tabular}

*Average based on Strongly Agree (5), Agree (4), Nuetral (3), Disagree (2), Strongly Disagree (1). 


\section{Chapter V}

\section{Discussion, Recommendations and Conclusions}

\section{Summary of Findings}

This chapter presents a summary of the current study, a discussion of the findings along with recommendations for future research based on these findings. This study aimed to determine whether a relationship existed between elementary preservice teachers' mathematical teacher knowledge development and participation in one of three mentor interaction structures during MLS. Growth in mathematical teacher knowledge, for this study, was investigated in terms of development in both mathematical content knowledge and pedagogical content knowledge. Additionally, this study undertook to investigate what aspects of the discourse during debriefing sessions, for the respective interaction structures, helped support development of elementary preservice teachers' mathematical teacher knowledge.

One hundred-three elementary preservice teachers enrolled in five sections of MAE 4310 voluntarily participated in the current study that included the completion of an MLS cycle according to one of three assigned interaction structures within three of the sections. Seventytwo participants completed MLS while enrolled in one of three sections of the course and an additional thirty-one elementary preservice teachers were enrolled in two different sections of MAE 4310 that did not complete MLS. All participants were enrolled in MAE 4310 during the spring semester of 2011 at Florida International University.

To measure growth in mathematical content knowledge, results from an initial preassessment, a second pre-assessment administered prior to beginning the MLS cycle, and a postassessment were analyzed. Participants in the comparison group completed only the initial preassessment and post-assessment. To measure growth in pedagogical content knowledge, group 
lesson plans submitted by participants as part of their MLS final report were analyzed. After completing the MLS cycle, participants completed a feedback survey to capture participants' perceptions of how completion of the experience contributed to their growth in mathematical teacher knowledge. Finally, transcripts of the discourse during debriefing sessions were analyzed and used to inform findings from the analysis of the development of mathematical teacher knowledge.

Results from the analysis for growth in mathematical content knowledge revealed that participants grew in their understanding of the mathematics measured by the assessment instrument irrespective of their assigned interaction structure. Additionally, participants who completed MLS in their respective course grew more in their knowledge of the mathematics measured by the assessment instrument when compared to those participants who did not complete MLS in their course. Results from the analysis for growth in pedagogical content knowledge with respect to Graeber's (1999) framework revealed the most growth for all big ideas occurred in the first interaction structure in which the MLS mentor was present during both the first and second debriefing sessions.

Analysis of the discourse during the debriefing sessions revealed four major themes were the subject of the discussions for all interaction structures. These themes included content knowledge, pedagogical content knowledge, MLS group issues, and lesson planning within the MLS structure. Interestingly, pedagogical content knowledge and lesson planning within the MLS structure were the two themes discussed most by all three interaction structures. However, the analysis of the lesson plans for growth in pedagogical content knowledge revealed significant growth for participants assigned to the first and second interaction structure, but little or no growth for those assigned to the third interaction structure. This suggests a higher quality of 
pedagogical content knowledge discussions for participants assigned to the first and second interaction structure, in which the MLS mentor was present.

Results of the feedback survey revealed participants in all interaction structures felt their content knowledge, pedagogical content knowledge, and lesson planning ability had increased as a consequence of their participation in the MLS process. Participants assigned to the first and second interaction structures indicated they would not have liked more feedback from their MLS mentor and valued the feedback they had received. In contrast, participants in the third interaction structure indicated they would have liked more feedback from their MLS mentor and did not find the feedback they had received helpful.

\section{Discussion of the Findings}

This study has several important implications for educators who intend to implement MLS either in school districts as a professional development process or within courses at the university level. The following is a discussion of each implication.

\section{Growth in Content Knowledge as a consequence of participation in MLS}

First, similar to the findings of M. Fernandez $(2005,2010)$ for secondary preservice teachers participating in MLS, elementary preservice teachers who completed MLS increased their understanding of the mathematics measured by the pre- and post-assessment instruments when compared to the non-MLS group, as well as separately. However, the comparison of the gain scores by interaction structure was not significant. Although the content knowledge growth of MLS participants appears to have occurred irrespective of mentor interaction structure, these results must be interpreted cautiously for multiple reasons. As stated previously, limitations related to the instruments used to measure growth in content knowledge may have skewed the findings. For example, the use of only one question per topic may not be sufficient to measure 
knowledge growth for that topic. Another issue that may have affected the outcome of the content knowledge findings was the choice to not vary topics across the interaction structures. It is possible that certain topics may have been more challenging than others, which would mean more growth would be possible within such concepts.

It is important to note that the analysis of the discourse of the MLS groups during debriefing sessions revealed participants who either had a misunderstanding or incorrect notion related to the content of their assigned topic tended to identify and clarify their issue in the presence of the MLS mentor. For example, during the first debriefing session with the multiplying fractions group, as the lesson tasks were discussed it became clear to the MLS mentor that the group had posed questions that were aimed at developing division of fractions rather than multiplying fractions. While other members of the group began to realize the confusion in the wording in the problems they had posed during the debriefing session, one member continued to struggle with this idea as seen in the following exchange:

Christy: What I mean by the dividing by multiplying is that our whole problem like the whole worksheet that we have is that we have half a cookie and we want to divide into like friends, right? So, we want to get a fraction of the cookie for each friend, right? So, we're dividing, but in the end like what we're doing is we're multiplying. So, I think that we need to tell them like okay, when you're dividing-- Like when you're dividing a fraction into more fractions that you're coming like that you end up kind of multiplying to get that final fraction that each persons going to get.

Lara: You want to change the whole idea of the--

Christy: Well, we don't really have to change it. I think we just have to, I guess, point it out. So like if we say "Okay, you have half a cookie, so you put one-half and you have four friends, like what fraction of the cookie will one person out of the four friends that you have get?"

MLS Mentor: Okay. So, maybe you want to change the wording to be more like what you're saying now?

Christy: Yes. 
Through the discussion with the MLS mentor and her other group members, eventually the participant began to resolve the issue. However, had the MLS mentor not been present it may have been that the group would have continued to use the problems they had posed in subsequent lessons. For example, during the third debriefing session of the comparing fractions group, the MLS mentor discovered that the group member that had taught the last lesson had not understood her topic as seen in the following excerpt:

Gina: No, I didn't have counters. I didn't understand the counters. I sucked at fractions. MLS Mentor: But no, no, no. Gilma, you have to understand the mathematics of your lesson before you go to teach your lesson.

Gina: Yes.

As the discussion continued, it became clear that the other members of the group, also, did not fully understand the lesson. Although, the MLS mentor helped to clarify the content issue for this group during the third debriefing session, this resolution did not take place until after all the lessons had been taught and the error had already been made. Thus, the presence of the MLS mentor during the earlier debriefing sessions is a valuable tool in helping to identify and resolve content issues before the lessons are taught and incorrect notions or ideas are passed onto to other elementary preservice teachers.

\section{Richer PCK discussions with MLS mentor}

While all participants grew in their understanding of pedagogical content knowledge, those participants assigned to the first interaction structure grew the most. Participants assigned to the second interaction structure showed moderate growth overall for pedagogical content knowledge, but some groups in this interaction structure regressed with respect to Graeber's (1999) big ideas. Participants assigned to the third interaction structure grew very little. Analysis of the discourse during debriefing sessions revealed a substantial portion of the 
discussions were related to pedagogical content knowledge indicating a difference in the quality of these discussions exists in the absence of the MLS mentor.

The contrast in the discussion quality between those MLS groups assigned to the different interaction structures indicates the MLS mentor is a key component in establishing richer and more reflective discourse during the debriefing sessions. It is possible that the richer discussions that occur when the MLS mentor was present are were a consequence of the group members reaching the Zone of Proximal Development, which according to Vygotsky (1999) is the place where the internalization of new ideas occurs. Furthermore, according to Vygotsky's sociocultural theory, individuals learn through social interaction with others. Thus, to operate in the Zone of Proximal Development, learners should be grouped so that members with a high understanding of a particular concept are mixed with those who possess a lesser understanding. Given that MLS groups are structured so that members are mixed in terms of their ability, the differences in gains amongst groups can be explained by the presence of the MLS mentor. The questions posed by the MLS mentor challenged participants ideas related to pedagogical content knowledge whereas the questions raised by the participants in the absence of the MLS mentor were related to superficial aspects of lessons.

For example, during the first debriefing session of the properties of quadrilateral group, in which the MLS mentor was present, group members expressed their views as to why the students in their lesson had not used the manipulatives they had provided. The group seemed to conclude that their students had not used the manipulatives because their students were their peers and not actual third grade students. The MLS mentor challenged their conclusion by asking if they had structured their lesson so as to require the use of manipulatives, see below:

Jan: I feel exactly the same. We were all discussing, we gave them a ruler and protractor so they are going to really use it. 
Moderator: Right

Jan: So because they did not take that, I mean they did not take it into mind that they are third graders so, if they know that it is a triangle, they just put it down, they did not actually go measure it.

Moderator: Measure it.

Jan: So we have to go about a different way but that we make sure that they use them.

Moderator: You could remind them next time, remember you guys are in the third grade class, and I will make a like a note to say you are supposed to behave but still you are third grade. But did you structure that, like did you direct them to use their rulers and protractors and stuff to measure things? Because if you just have it out there and did not tell them to use it...

The MLS mentor's question pushed the group members to consider whether they had developed a lesson that provided the opportunity for their students to use the manipulatives. These group members along with their MLS mentor operated in the Zone of Proximal Development. Thus, the elementary preservice teachers' were sufficiently challenged by the questions posed by the MLS mentor so as to allow for growth and development in their ideas. The interaction described with this example was typical of the debriefing sessions that included the MLS mentor. In contrast, debriefing sessions that did not include the MLS mentor tended to include discourse that was more descriptive of the lesson. For example, the following is an excerpt from the first debriefing session for the comparing fractions group, in which the MLS mentor was not present:

Daniella: ...The first thing that Michelle did in the lesson was that she explained what a fraction is and then after that she demonstrated how to simplify a fraction. Once she finished explaining how one can simplify a fraction, she explained to the class that they were going to be comparing fractions using fraction strips. And then, she passed out a handout in which each problem had two fractions. 
The excerpt presented above was representative of the remainder of this debriefing session. No group member posed questions to challenge the structure of the lesson and so the group members were unable to reach the Zone of Proximal Development.

A second contributing factor to the discrepancy in the quality of the discussions between elementary preservice teachers assigned to the first and third interaction structures may have been the participants concern for their group members' feelings. Based on the feedback survey results, participants' assigned to the third interaction structure had a slightly higher rating when asked whether their concern for their group members' feelings was a factor in their assessment and evaluation of their peers' lesson. The presence of the MLS mentor during the first and second debriefing sessions may have established a precedence for how to discuss a lesson while remaining impartial.

The importance of the MLS mentor in facilitating preservice teachers' learning during the MLS process is aligned with findings from M. Fernandez (2010) and M. Fernandez and Zilliox (2011). However, the present study suggests that the extent of the interaction of the MLS mentor with the elementary preservice teachers is an essential consideration. Elementary preservice teachers assigned to the second interaction, in which the MLS mentor was present only during the first debriefing session, either grew minimally or regressed in terms of their pedagogical content knowledge from the first lesson to the third lesson. This appears to be in contrast with findings from M. Fernandez (2010), who found substantive growth in secondary mathematics teachers' ability to plan lessons that were student-centered given only one formal meeting with the MLS mentor during their first or second MLS group debriefing sessions. However, the MLS mentor in M. Fernandez (2010) was available as a resource and interacted with the participants throughout the MLS experience, not only the formal meeting; she "observed and watched 
videotapes of the MLS lessons and interacted with the MLS groups in and out of class, sometimes providing written questions for the groups to consider" (p. 354) and "engaged MLS group members in brief discussions about their group cooperation in order to support their efforts to cooperate effectively" (p. 354).

The present study with elementary preservice teachers confirms the importance of the MLS mentor as part of the MLS experience and the need for the MLS mentor to help preservice teachers think more deeply about their teaching and student learning of mathematics. For a mentor to strictly meet during one or no debriefing sessions with a MLS group does not sufficiently facilitate preservice teacher learning. Structuring the MLS experience so that the MLS mentor formally meets each group during two debriefing meetings adds a level of accountability for the MLS mentor to interact with and monitor the progress of each group across the experience. However, formally meeting with the MLS groups during their first or second debriefing and continual monitoring and interacting with the groups or individual participants throughout the experience may provide comparable support for the preservice teachers' learning. However, more research is needed to investigate this possibility.

\section{Value of formative vs. summative interactions with MLS mentor}

Analysis of the discourse also revealed that groups assigned to the first and second interaction structures who encountered issues either with the mathematics of their lesson, planning the lesson or with group collaboration tended to resolve their issues early in the MLS process. In contrast, groups assigned to the third interaction structure tended to struggle with these issues until the third debriefing session in which the MLS mentor was present. Additionally, results of the feedback survey revealed elementary preservice teachers assigned to the third interaction structure would have liked more feedback from the MLS mentor as well as 
did not value the feedback they received as much as the preservice teachers assigned to the first and second interaction structures. This suggests the formative feedback provided by the MLS mentor in the first and second interaction structures was more valued by elementary preservice teachers than summative feedback. This is in alignment with the ideas of M. Fernandez and Zilliox (2011) who noted the importance of the MLS mentor in providing formative feedback during the lesson study cycles.

\section{Insights related to Graeber's (1999) five big ideas}

Another informative finding from this study is the lack of discussion during debriefing sessions related to two of Graeber's (1999) five big ideas. These two big ideas were students knowing in one way do not necessarily know in the other(s) and intuitive knowledge is both an asset and a liability. This finding may explain the moderate to minimal growth found in these areas during the analysis of the lesson plans. This suggests one possibility to help improve growth with respect to these two big ideas is to request the MLS mentor make a concerted effort to address all of Graeber's five big ideas during the debriefing sessions.

Also, through the completion of this study, a rubric for analyzing lesson plans, see Appendix D, was developed based on Graeber's (1999) framework for pedagogical content knowledge. This rubric concretizes Graber's framework in ways that may be useful for researchers, project evaluators, mathematics educators, professional development facilitators and others that seek to use lesson plans to record individuals' levels of mathematical pedagogical content knowledge or assess growth in individuals' mathematical pedagogical content knowledge while partaking in professional learning experiences and projects. Through the use of the rubric, preservice and practicing teachers may also reflect on their use of pedagogical content 
knowledge in their own lessons and assess their own ability to plan mathematics lessons that are rich in terms of pedagogical content knowledge.

\section{Recommendations for Future Research}

In view of the findings resulting from this study, recommendations for future research can be made. First, given the inconclusive results related to the content knowledge piece of this study, a new study might investigate MLS participants' growth in this area with the same varied interaction structures, but using an improved instrument to measure gains in content knowledge. Additionally, a similar study might vary the topics amongst the interaction structures to better understand whether the use of the same topics in this study played a role in the outcome of the content analysis.

Next, future research might investigate whether similar findings related to content and pedagogical content knowledge result when the interaction structure is varied with secondary mathematics preservice teachers or practicing teachers as well as other disciplines such as science. Also, researchers might investigate other aspects of the MLS process besides the discourse during debriefing sessions that might contribute to growth in mathematical teacher knowledge such as in-class planning time and work participants conduct out-of-class related to the MLS.

Finally, a longitudinal study could be conducted to follow elementary preservice teachers into their classrooms as they become teachers. It would be interesting to investigate their ability to lesson plan after completing MLS. Also, it would valuable to investigate whether differences exist in their students' learning when compared to teachers who have not participated in MLS. 


\section{Conclusions}

This study found that participation in the MLS process helped to increase elementary preservice teachers' mathematical teacher knowledge irrespective of their assigned interaction structure. However, elementary preservice teachers assigned to the first interaction structure, in which the MLS mentor participated in two of the three debriefing sessions, grew more in their understanding of pedagogical content knowledge, tended to resolve group issues with mathematics, pedagogy, and collaboration earlier, and valued the experience more than elementary preservice teachers assigned to the other interaction structures. This suggests the formative feedback and presence of the MLS mentor during the first two debriefing sessions was an important factor in the development of the mathematical teacher knowledge of the elementary preservice teachers who participated in this study during the MLS process. However, as these results are based on work with elementary preservice teachers, future research might investigate the outcome of the MLS process with varied interaction structures with secondary preservice teachers or practicing teachers. 


\section{References}

Burkhardt, H. \& Schoenfeld, A.H. (2003). Improving educational research: Toward a more useful, more influential, and better-funded enterprise. Educational Researcher, 32(9), 314.

Cochran-Smith, M. (1999) Learning to teach for social justice. In G. Griffin (Ed.), The Education of Teachers: The Ninety-eighth yearbook of the national society for the study of education (pp. 114-144). Chicago: University of Chicago Press.

Darling-Hammond, L. (2001). Successful teacher education programs in the era of reform: An essay review of studies of excellence in teacher education. Teaching and Teacher Education, 17(5), 645-651.

Fernandez, C., Cannon, J., \& Chokshi, S. (2003). A US-Japan lesson study collaboration reveals critical lenses for examining practice. Teaching and Teacher Education, 19(2), 171-185.

Fernandez, M.L. (2005). Learning through microteaching lesson study in teacher preparation. Action in Teacher Education, 26(4), 37-47.

Fernandez, M. L. (2006). Prospective teachers' perspectives on microteaching lesson study. Education, 127(2), 203-215.

Fernandez, M. L. (2010). Investigating how and what prospective teachers learn through microteaching lesson study. Teaching and Teacher Education, 26 (2), 351-562.

Fernandez, M. L. \& Zilliox, J. (2011). "Approaches to lesson study in prospective teacher education.” In L. Hart, A. Alston, \& A. Murata (Eds.), Lesson study across mathematics education contexts (85-102). London: Springer Inc.

Gall, M.D., Gall, J.P., \& Borg, W.R. (2007). Educational Researcher: An Introduction: (Eighth Edition). Boston: Pearson.

Graeber, A.O. (1999). Forms of knowing mathematics: What preservice teachers should learn. Educational Studies in Mathematics, 38(1), 189-208.

Hesse-Biber, S.N. (2010). Mixed methods research: Merging theory with practice. New York, NY: The Guilford Press.

Hinkle, D.E., Wiersma, W., \& Jurs, S.G. (2003). Applied statistics for the behavioral sciences: (Fifth Edition). Boston: Houghton Mifflin.

Lewis, C., Perry, R., \& Hurd, J. (2004). A deeper look at lesson study. Educational Leadership, 61(5), 18-22.

Lewis, C., Perry, R., \& Murata, A. (2006). How should research contribute to instructional 
improvement? The case of lesson study. Educational Researcher, 35(3), 3-14.

Matthews, M., Hlas, C.S., \&Finken, T.M. (2009). Using lesson study and four-column lesson planning with preservice teachers: Lessons from lessons. Mathematics Teacher, 102(7), 504-509.

McMahon, M.T. \& Hines, E. (2008). Lesson Study with Preservice Teachers. Mathematics Teacher, 102(3), 186-191.

Menon, R. (2000). Should the United States emulate Singapore's education system to achieve Singapore's success in the TIMSS? Mathematics Teaching in the Middle School. 5(6), 345-347.

Miles, M.B. \& Huberman, A.M. (1994). Early steps in analysis. In Qualitative Data Analysis: An Expanded Sourcebook ( $2^{\text {nd }}$ Edition).Thousand Oaks: Sage.

National Center for Education Statistics. (1999 and 2007). Trends in International Mathematics and Science Study. Retrieved from http://nces.ed.gov/timss/results.asp

National Mathematics Advisory Panel. (2008). Foundations for success: The final report of National Mathematics Advisory Panel. Washington, DC: U.S. Department of Education.

Paige, R. (2002, November 18). Opening remarks to the Forum on Rigorous Evidence: The key to progress in education? Lessons from medicine, welfare, and other fields. Retrieved December 23, 2004, from http://www.excelgov.org

Parks, A.N. (2007). Messy learning: Preservice teachers' lesson study conversations about mathematics and students. Teaching and Teacher Education, 24(5), 1200-1216.

Post, G., \&Varoz, S. (2008). Lesson-study groups with prospective and practicing teachers. Teaching Children Mathematics, 14(8), 472-478.

Puchner, L.D. \& Taylor, A.R. (2006). Lesson study, collaboration and teacher efficacy: Stories from two school-based math lesson study groups. Teaching and Teacher Education, 22(7), 922-934.

Shulman, L.S. (1986). Those who understand knowledge growth in teaching. Educational Researcher, 15(2), 4-14.

Sims, L., \& Walsh, D. (2008). Lesson study with preservice teachers: Lessons from lessons. Teaching and Teacher Education, 25(5), 724-733.

Stigler, J.W. \& Hiebert, J. (1999). The teaching gap: Best ideas from the world's teachers for improving education in the classroom. New York: The Free Press.

Takahashi, A. \& Yoshida, M. (2004). Ideas for Establishing Lesson-Study Communities. 
Teaching Children Mathematically, 10(9), 436-443.

Taylor, A.R., Anderson, S., Meyer, K., Wagner, M.K., \& West, C. (2005). Lesson study: A professional development model for mathematics reform. The Rural Educator, 26(2), 1722.

Van Hiele, P.M. (1959). The Child's Thought and Geometry. In D. Fuys, D. Geddes, \& R. Tischler (Eds.) (1959/1985): English Translation of Selected Writings of Dina van HieleGeldof and Pierre M. van Hiele, (pp. 243-52). ERIC/SMEAC

Vygotsky, L. (1978). Mind in society (M. Cole, S. Scribner, V. John-Steiner, \& E. Souberman, Trans.). Cambridge, MA: Harvard University (Original work published 1934). 


\section{APPENDICES}

Appendix A: Pre/Post-Assessment 
Name:

Group \#: Date:

Group Topic: Group members:

\section{MLS Pre/Post-assessment}

1. Solve the following problem using two different strategies:

Tommy was on page 48 of a book he was reading. If he reads 65 more pages tomorrow, what page of the book will he be on tomorrow?

2. Use a diagram to solve the following problem:

Jack and Jill shared a pizza. Jack ate $\frac{2}{3}$ of a pizza. Jane ate $\frac{1}{6}$ of the pizza. How much of the pizza did they eat together? 
3. Use a fraction model to solve the following problem:

Laura took $\frac{2}{5}$ of a batch of cookies to school. Mark ate $\frac{1}{4}$ of the cookies Laura brought to school. How much of the original batch of cookies did Mark eat?

4. Using a diagram, explain how you would compare $\frac{4}{5}$ and $\frac{7}{8}$. 
5. Give the formulas for the area of each polygon:
a. triangle
b. parallelogram
c. rectangle
d. trapezoid

6. Explain how the formulas in question 5 are related.

7. Shade 12 boxes in the grid below.

\begin{tabular}{|l|l|l|l|l|l|l|l|l|l|}
\hline & & & & & & & & & \\
\hline & & & & & & & & & \\
\hline & & & & & & & & & \\
\hline & & & & & & & & & \\
\hline
\end{tabular}

a. What fraction represents the shaded region in the grid?

b. What percent represents the shaded region in the grid?

c. What decimal represents the shaded region in the grid? 
8. Using base ten models, show three equivalent representations of 486 .

9. Make a drawing to solve the following problem:

A bag has 872 jelly beans. Michael and his three friends want to share them equally. How many jelly beans will Michael and each of his friends get? 
10. Create a table that illustrates the properties of the following quadrilaterals: square, rectangle, parallelogram, trapezoid, kite, and rhombus.

11. List two pairs of numbers that will satisfy the following equation:

$$
2+\square=\square+5
$$


Appendix B: Microteaching Lesson Study Report Guidelines 
Micro-teaching Lesson Study Group Project (Adapted from the work of M. Fernandez $(2005,2010)$,

Process Goal for project lesson is to develop students' mathematical reasoning and ability to study patterns in constructing relationships or concepts through experimenting, analyzing, conjecturing, and defending or justifying mathematical ideas. (Construct-a-Concept or Discovera-Relationship lessons)

\section{Project Report Sections}

Include videotapes of all the lessons with the final project. Follow lesson format given and discussed in class.

\section{$\underline{\text { Section I }}$}

(a) Type pre-lesson thoughts: What dilemmas or difficulties is your group facing with regard to this lesson and its implementation? What lesson related goals does your group have for the students? What student responses, comments, or questions will your group look for as evidence of understanding and achieving lesson goals and objectives.

(b) First Lesson Plan Draft with all supporting materials and handouts.

(c) Videotape of first teaching (approx. 20-30 min)

(d) Teachers watch videotape and each completes an Individual Group Member's VideoLesson Analysis (MSWord version will be for electronic completion as part of report).

\section{$\underline{\text { Section II }}$}

(a) Summary of Observations and Revisions on first teaching for creating Second Lesson Draft: As a group, discuss your observations about the First Teaching of the Lesson including what were the strengths and weaknesses of the lesson particularly with respect to student learning and at least in part in relation to Professional Mathematics Teaching Standards. Type a summary of your group observations and what your group will change the second time the lesson is taught. Be sure to explain why your group is making the changes. Include any new materials or handouts to be used behind the second lesson draft.

(b) Second Lesson Plan Draft with all supporting materials and handouts.

(c) Videotape of second teaching (approx. 20-30 min)

(d) Teachers watch videotape and each completes an Individual Group Member's VideoLesson Analysis (MSWord version will be for electronic completion as part of report).

\section{Section III}

(a) Summary of Observations and Revisions on second teaching for creating Third Lesson Draft: As a group, discuss your observations about the Second Teaching of the Lesson including what were the strengths and weaknesses of the lesson particularly with respect to student learning and at least in part in relation to Professional Mathematics Teaching Standards. Type a summary of your group observations and what your group will change the third time the lesson is taught. Be sure to explain why your group is making the changes. Include any new materials or handouts to be used behind the third lesson draft.

(b) Third Lesson Plan Draft with all supporting materials and handouts.

(c) Videotape of first teaching (approx. 20-30 min) 
(d) Teachers watch videotape and each complete an Individual Group Member's VideoLesson Analysis (MSWord version will be for electronic completion as part of report). Section IV

(a) Summary of Observations and Revisions on third teaching to create final Revised Lesson Plan: As a group, discuss your observations about the Third Teaching of the Lesson including what were the strengths and weaknesses of the lesson particularly with respect student learning and at least in part in relation to Professional Mathematics Teaching Standards. Type a summary of your group observations and what your group will change for the final revised lesson. Be sure to explain why your group is making the changes. Indicate any final

adjustments your group would make to the lesson? Explain why? Include any new materials or handouts behind the final revision lesson plan.

(b) Final Revision Lesson Plan with all supporting materials and handouts.

(c) Suggestions for Teaching the Lesson

Not included in Project will be in class Assessment of Collaboration

As you work together as a group be sure to participate in the following ways:

(1) Contribute ideas to group goal (MLS lessons, MLS report, MLS presentation).

(2) Encourage everyone's participation.

(3) Met all group responsibilities (e.g., timely work, meetings).

(4) Percent contributed to group work (Each member should work toward participating equally) 
Appendix C: Feedback Survey 
Name:

Group \# Date:

Group Topic:

Group members:

Feedback on Micro-Teaching Project (Adapted from the work of M. Fernandez (2005, 2006, 2010)),

(1) What were the two most important things you learned through the Micro-Teaching Study (MLS) Project? Explain why they were important.

(2) (a) How might you develop the relationship between mixed numbers and improper fractions for the first time with your students?

(b) Explain why you would choose the approach in 2(a). 
(3) (a) How might you teach students learning for the first time about adding decimals?

(b) Explain why you would choose the approach in 3(a).

(4) What did you learn about your mathematics topic through the MLS? Be specific.

(5) What did you do to deepen your knowledge of your topic, if anything? Be specific. 
(6) As a teacher, what can you do to develop your own knowledge of the mathematical topic you will be teaching?

For each of the following circle one of the ratings and explain your reasoning.

(7) Planning together with other group members helped me broaden my knowledge of possible ways of teaching the desired lesson.

\begin{tabular}{|c|c|c|c|c|}
\hline Strongly Agree & Agree & Neutral & Disagree & $\begin{array}{c}\text { Strongly } \\
\text { Disagree }\end{array}$ \\
\hline
\end{tabular}

Explain:

(8) Analyzing each other's teaching of the lesson helped me think more deeply about my own teaching.

\begin{tabular}{|c|c|c|c|c|}
\hline Strongly Agree & Agree & Neutral & Disagree & $\begin{array}{c}\text { Strongly } \\
\text { Disagree }\end{array}$ \\
\hline
\end{tabular}

Explain: 
(9) Feedback from my group members helped me understand my teaching strengths and areas for improvement.

\begin{tabular}{|c|c|c|c|c|}
\hline Strongly Agree & Agree & Neutral & Disagree & $\begin{array}{c}\text { Strongly } \\
\text { Disagree }\end{array}$ \\
\hline
\end{tabular}

Explain:

(10) When I analyzed my group members' teaching of the lesson, my concern for their feelings influenced my assessment and feedback.

\begin{tabular}{|c|c|c|c|c|}
\hline Strongly Agree & Agree & Neutral & Disagree & $\begin{array}{c}\text { Strongly } \\
\text { Disagree }\end{array}$ \\
\hline
\end{tabular}

Explain:

(11) Planning together with other group members helped me deepen my knowledge of the mathematics topic we taught.

\begin{tabular}{|c|c|c|c|c|}
\hline Strongly Agree & Agree & Neutral & Disagree & $\begin{array}{c}\text { Strongly } \\
\text { Disagree }\end{array}$ \\
\hline
\end{tabular}

Explain: 
(12) The actual teaching of the lesson helped me deepen my understanding of the mathematics topic.

\begin{tabular}{|c|c|c|c|c|}
\hline Strongly Agree & Agree & Neutral & Disagree & $\begin{array}{c}\text { Strongly } \\
\text { Disagree }\end{array}$ \\
\hline
\end{tabular}

Explain:

(13) Preparing to teach this topic and planning the lesson caused me to engage in mathematical reasoning and problem solving.

\begin{tabular}{|c|c|c|c|c|}
\hline Strongly Agree & Agree & Neutral & Disagree & $\begin{array}{c}\text { Strongly } \\
\text { Disagree }\end{array}$ \\
\hline
\end{tabular}

Explain:

(14) Input from the MLS Mentor (ie., instructor) was helpful as we designed our lesson.

\begin{tabular}{|c|c|c|c|c|}
\hline Strongly Agree & Agree & Neutral & Disagree & $\begin{array}{c}\text { Strongly } \\
\text { Disagree }\end{array}$ \\
\hline
\end{tabular}

Explain: 
(15) Input from the MLS Mentor (ie., instructor) was helpful in understanding the content of our lesson.

\begin{tabular}{|c|c|c|c|c|}
\hline Strongly Agree & Agree & Neutral & Disagree & $\begin{array}{c}\text { Strongly } \\
\text { Disagree }\end{array}$ \\
\hline
\end{tabular}

Explain:

(16) I would have liked to have more input or feedback on our group lesson from the MLS Mentor (ie., instructor) during the teaching and analysis phases of the project.

\begin{tabular}{|c|c|c|c|c|}
\hline Strongly Agree & Agree & Neutral & Disagree & $\begin{array}{c}\text { Strongly } \\
\text { Disagree }\end{array}$ \\
\hline
\end{tabular}

Explain:

(17) I would not like to engage in MLS in other contexts (ie. other courses or as a practicing teacher).

\begin{tabular}{|c|c|c|c|c|}
\hline Strongly Agree & Agree & Neutral & Disagree & $\begin{array}{c}\text { Strongly } \\
\text { Disagree }\end{array}$ \\
\hline
\end{tabular}

\footnotetext{
Explain:
} 
(18) Teaching strategies I learned through MLS will be useful when I teach elementary school students.

\begin{tabular}{|c|c|c|c|c|}
\hline Strongly Agree & Agree & Neutral & Disagree & $\begin{array}{c}\text { Strongly } \\
\text { Disagree }\end{array}$ \\
\hline
\end{tabular}

Explain:

(19) MLS deepened my ability to anticipate student responses to mathematical questions and tasks.

\begin{tabular}{|c|c|c|c|c|}
\hline Strongly Agree & Agree & Neutral & Disagree & $\begin{array}{c}\text { Strongly } \\
\text { Disagree }\end{array}$ \\
\hline
\end{tabular}

Explain:

(20) Other comments or suggestions (continue on back as needed): 
Appendix D: Pre/Post Assessment Rubric 
Pre/Post Assessment Rubric

\begin{tabular}{|c|c|c|c|}
\hline Question & Points & Description* & Total Points \\
\hline \multirow{3}{*}{1} & 0 & Answer incorrect & \multirow{3}{*}{2} \\
\hline & 1 & Used 1 strategy and answer correct $(\mathrm{P})$ & \\
\hline & 1 & Used 2 strategies and answer correct (C) & \\
\hline \multirow{3}{*}{2} & 0 & Answer incorrect & \multirow{3}{*}{2} \\
\hline & 1 & Answer correct $(\mathrm{P})$ & \\
\hline & 1 & Diagram correct $(\mathrm{C})$ & \\
\hline \multirow{3}{*}{3} & 0 & Answer incorrect & \multirow{3}{*}{2} \\
\hline & 1 & Answer correct $(\mathrm{P})$ & \\
\hline & 1 & Fraction model correct $(\mathrm{C})$ & \\
\hline \multirow{3}{*}{4} & 0 & Answer incorrect & \multirow{3}{*}{2} \\
\hline & 1 & Answer correct $(\mathrm{P})$ & \\
\hline & 1 & Diagram correct $(\mathrm{C})$ & \\
\hline \multirow{5}{*}{$5(P)^{* *}$} & 0 & No formula is correct & \multirow{5}{*}{2} \\
\hline & 0.5 & One formula is correct & \\
\hline & 1 & Two formulas are correct & \\
\hline & 1.5 & Three formulas are correct & \\
\hline & 2 & Four formulas are correct & \\
\hline \multirow{4}{*}{$6(C)^{* *}$} & 0 & No relationship is clearly explained & \multirow{4}{*}{2} \\
\hline & 1 & One relationship is clearly explained & \\
\hline & 1.5 & Two relationships are clearly explained & \\
\hline & 2 & All relationships are clearly explained & \\
\hline \multirow{4}{*}{$7(P)$} & 0 & No representation is correct & \multirow{4}{*}{2} \\
\hline & 1 & One representation is correct & \\
\hline & 1.5 & Two representations are correct & \\
\hline & 2 & All representations are correct & \\
\hline \multirow{3}{*}{8} & 0 & No representation is correct & \multirow{3}{*}{2} \\
\hline & 1 & One representation is correct $(\mathrm{P})$ & \\
\hline & 1 & Two or three representations are correct $(\mathrm{C})$ & \\
\hline \multirow{3}{*}{9} & 0 & Answer incorrect; Divided or shared with 3 only & \multirow{3}{*}{2} \\
\hline & 1 & Answer correct $(\mathrm{P})$ & \\
\hline & 1 & Drawing correct $(\mathrm{C})$ & \\
\hline \multirow{7}{*}{$10(P)$} & 0 & No Minimum Defining List (MDL) is correct & \multirow{7}{*}{3} \\
\hline & 0.5 & One MDL is correct & \\
\hline & 1 & Two MDLs are correct & \\
\hline & 1.5 & Three MDLs are correct & \\
\hline & 2 & Four MDLs are correct & \\
\hline & 2.5 & Five MDLs are correct & \\
\hline & 3 & Six MDLs are correct & \\
\hline \multirow{3}{*}{11} & 0 & No pair of numbers is correct & \multirow{3}{*}{2} \\
\hline & 1 & One pair of numbers is correct $(\mathrm{P})$ & \\
\hline & 2 & Two pairs of numbers are correct (C) & \\
\hline
\end{tabular}

*P represents procedural understanding and $\mathrm{C}$ represents conceptual understanding

**Questions 5 and 6 are related to the same topic. Question 5 is the procedural piece of the topic and Question 6 is the conceptual piece of the topic. 
Appendix E: Pedagogical Content Knowledge Rubric 


\section{Rubric for Pedagogical Content Knowledge Analysis Using Graeber's Framework}

\begin{tabular}{|c|c|c|}
\hline Big Idea & Code & Description \\
\hline \multirow{3}{*}{$\begin{array}{l}\text { Understanding students' current } \\
\text { understanding }\end{array}$} & Low & $\begin{array}{l}\text { Lesson plan reflects teacher's attempt to assess current student understanding, but it is not } \\
\text { connected to moving the students ideas forward or the attempt is not sufficiently relevant } \\
\text { to goal of lesson }\end{array}$ \\
\hline & Medium & $\begin{array}{l}\text { Lesson plan reflects teacher's attempt to assess current student understanding, and is } \\
\text { connected to moving student ideas forward in a limited way or when applicable, does not } \\
\text { appear to be grounded in research-based stages of development for a particular topic }\end{array}$ \\
\hline & High & $\begin{array}{l}\text { Lesson plan reflects teacher's knowledge of the stages (research based when applicable) of } \\
\text { student's development associated with particular topics and consideration is made within } \\
\text { the lesson to assess student understanding within such hierarchies with the intent to move } \\
\text { students forward in their development of the topic }\end{array}$ \\
\hline \multirow{3}{*}{$\begin{array}{l}\text { Students knowing in one way do not } \\
\text { necessarily know in the other(s) }\end{array}$} & & Lesson plan reflects development of procedural understanding only \\
\hline & & $\begin{array}{l}\text { Lesson plan reflects development of procedural understanding and an attempt is made to } \\
\text { develop conceptual understanding, but it is limited or does not completely develop the } \\
\text { mathematical idea }\end{array}$ \\
\hline & & $\begin{array}{l}\text { Lesson plan reflects development of conceptual understanding through mathematically } \\
\text { rich experiences and procedural understanding when appropriate }\end{array}$ \\
\hline \multirow{3}{*}{$\begin{array}{l}\text { Intuitive knowledge is both an asset } \\
\text { and a liability }\end{array}$} & & $\begin{array}{l}\text { Lesson plan reflects some knowledge of common preconceptions and misconceptions, but } \\
\text { addresses such notions in ways that fail to develop the correct mathematical idea }\end{array}$ \\
\hline & lium & $\begin{array}{l}\text { Lesson plan reflects knowledge of common preconceptions and misconceptions, but } \\
\text { planned ways to address such notions represent limited development of the correct } \\
\text { mathematical idea }\end{array}$ \\
\hline & & $\begin{array}{l}\text { Lesson plan reflects teacher's knowledge of common preconceptions and misconceptions } \\
\text { through inclusion of carefully planned tasks and responses intended to address such } \\
\text { notions in appropriate ways }\end{array}$ \\
\hline \multirow{3}{*}{$\begin{array}{l}\text { Certain instructional characteristics } \\
\text { appear to promote retention }\end{array}$} & & Lesson plan tasks involve teacher-directing or telling of mathematical ideas \\
\hline & & $\begin{array}{l}\text { Lesson plan tasks involve teacher-guiding the development of mathematical ideas or } \\
\text { attempts to have students work collaboratively but does so ineffectively or does not } \\
\text { require explanations of answers }\end{array}$ \\
\hline & High & $\begin{array}{l}\text { Lesson plan tasks actively involve students in development of their own mathematical } \\
\text { ideas, possibly through collaborative investigations or explorations, and requires } \\
\text { explanations of answers }\end{array}$ \\
\hline \multirow{3}{*}{$\begin{array}{l}\text { Alternative representations and the } \\
\text { recognition and analysis of } \\
\text { alternative methods are important }\end{array}$} & Low & Lesson plan reflects the use of only one mathematical representation \\
\hline & Medium & Lesson plan reflects the use of an alternative mathematical representation \\
\hline & High & $\begin{array}{l}\text { Lesson plan reflects the use of multiple mathematical representations or all appropriate } \\
\text { mathematical representations are used }\end{array}$ \\
\hline
\end{tabular}


Appendix F: Descriptive Codes for Discourse Analysis 
Descriptive Codes for Discourse Analysis:

Reflecting on/Discussing Lesson

Constuctivist Approach

Tasks/lesson structure

Student Engagement

Mathematics

Mathematical Representations

Group Collaboration Issues

Clarifying/Lack of Group Understanding

for

Mathematics

Ways to Get Help in Mathematics

Student Understanding

MLS Process/Group Progress/Grades

Teacher-Directed Instruction

Confidence/Nervous

Questions Posed in Lesson

Not authentic

Lack of Student Understanding
MLS Mentor Actions

Asking/Discussing Question about

Mathematics

Asking Question/Discussing taking constructivist approach

Asking Questions/Discussing tasks/lesson

structure

Asking Questions/Discussing about

student engagement

Asking Questions/Discussing about

mathematical representations

Asking Questions/Discussing about

Student Understanding

Encouraging group discussion

Mediating group collaboration issues

Discussing/Suggesting Ways to Find Help

to Understand Mathematics

Clarifying Mathematics

Discussing Student Solutions

Commenting on Tasks/Lesson Structure

Commenting on Student Engagement

Commenting on Questioning/Preparing

Questions

Commenting on Mathematics

Commenting/Discussing MLS

Process/Group Progress/Grades/Logistics

Commenting on Teacher-Directed

Instruction

Commenting on Ways to be Constructivist

Commenting on Mathematical

Representations

Commenting on Examples

Commenting on Not Authentic 
Appendix G: Discourse Analysis Themes 


\section{Discourse Analysis Themes}

\section{Theme \#1: Content Knowledge Discussion}

Asking/Discussing Question about Mathematics

Asking/Discussing Student Solutions

Commenting on Questioning/Preparing Questions

Commenting on Mathematics

Commenting on Examples

\section{Theme \#2: Pedagogical Content Knowledge Discussions}

Big Idea \#1:Understanding students' current understanding

- Asking Questions/Discussing about student engagement

- Asking Questions/Discussing about Student Understanding

- Commenting on Student Engagement

Big Idea \#2:Students knowing in one way do not necessarily know in the other(s)

- Asking Questions/Discussing Conceptual Understanding

Big Idea \#3:Intuitive knowledge is both an asset and a liability

- Asking Questions/Discussing Student Misconceptions or Errors

Big Idea \#4:Certain instructional characteristics appear to promote retention

- Asking Question/Discussing taking constructivist approach

- Asking Questions/Discussing Teacher-directed instruction

- Commenting on Teacher-Directed Instruction

- Commenting on Ways to be Constructivist

Big Idea \#5:Alternative representations and the recognition and analysis of alternative methods are important

- Asking Questions/Discussing about mathematical representations

- Commenting on Mathematical Representations

\section{Theme \#3: MLS Group Issues}

Group Collaboration Issues

Clarifying Pedagogy

Confidence/Nervousness

Professor Assistance

Content Issue Realization

\section{Theme \#4: Lesson Planning within MLS Structure}

Asking Questions/Discussing tasks/lesson structure

Asking Questions/Discussing Lesson Planning

Encouraging group discussion

Discussing/Suggesting Ways to Find Help to Understand Mathematics

Commenting on Tasks/Lesson Structure

Commenting/Discussing MLS Process/Group Progress/Grades/Logistics

Commenting on Not Authentic 


\section{VITA}

ROXANNE V. MOLINA

\section{EDUCATION}

PH.D. Florida International University Curriculum and Instruction 8/08-4/12

Dissertation Title: Microteaching Lesson Study: Mentor Interaction Structure and its Relation to Elementary Preservice Mathematics Teacher Knowledge Development

M.S. Florida International University Mathematics Education 8/01-8/06

B.S. Florida International University Mathematics \& Honors College 8/95-4/00

\section{PART-TIME ACADEMIC EXPERIENCE}

Florida International University $\quad$ Graduate Assistant $\quad$ Miami, FL 8/08-8/11

- Developed and taught curriculum that incorporated Blackboard as an instructional tool for MAE 4310 (Content and Methods of Teaching Elementary Mathematics), MAE 3651/5655 (Learning Mathematics with Technology), and MAE 6318 (Instruction in Elementary Mathematics).

- Assisted in data collection and analysis and research dissemination for research studies including Algebra Project, Contextual vs. Non-Contextual Problem Solving, and Microteaching Lesson Study

- Assisted in development of IRB Proposals

- Assisted in preparation of Grant Proposal

- Completed TESOL training to teach TESOL infused courses including MAE 4310

Spalding University $\quad$ Adjunct Instructor $\quad$ Louisville, KY 8/07-8/08

- Developed curriculum for and taught College Algebra and Statistical Methods for sixweek accelerated courses designed to meet the needs of non-traditional students.

\section{PUBLICATIONS IN DISCIPLINE}

Articles

Molina, R. \& Fernandez, M. L. (under review). Improving Mathematical Teacher Knowledge through Microteaching Lesson Study (MLS). Dimensions. (peer reviewed)

Proceedings

Molina, R., Fernandez, M.L. \& Nisbet, L. (2011). Analyzing Elementary Preservice Teachers' Development of Content and Pedagogical Content Knowledge in Mathematics through Microteaching Lesson Study. In T. Rocco (Ed.) Proceedings of the $10^{\text {th }}$ Annual College of Education and Graduate Student Network Research Conference. Miami, FL. (peer review) 


\section{PRESENTED PAPERS AND LECTURES}

Molina, R. \& Fernandez, M.L., Examining Elementary Preservice Teachers' Mathematical Teacher Knowledge Development through Participation in Microteaching Lesson Study $(M L S)$, Annual Meeting of the American Educational Research Association, Vancouver, British Columbia, Canada, Apr. 2012.

Molina, R. Nisbet, L. \& Fernandez, M. L., Examining the Effectiveness of Microteaching Lesson Study with Elementary Preservice Teachers, Annual Meeting of the Association of Mathematics Teacher Educators, Irvine, CA, Jan. 2011.

Nisbet, L., Molina, R. \& Fernandez, M. L., Preservice Teachers Problem Solving Strategies and Math Anxiety in Relation to Contextual vs. Non-contextual Problems, Annual Meeting of the Association of Mathematics Teacher Educators, Irvine, CA, Jan. 2011.

Molina, R. \& Fernandez, M. L., The Effect of Microteaching Lesson Study on Elementary Preservice Teacher Content and Pedagogical Content Knowledge, Annual Meeting of the Florida Educational Research Association, Orlando, FL, November 2010. (refereed)

Molina, R., Nisbet, L., \& Fernandez, M. L. Preservice Teachers' Problem Solving Strategies and Math Anxiety in Relation to Contextual vs. Non-Contextual Problems, Annual National Council of Teachers of Mathematics Regional Meeting, New Orleans, LA, October 2010.

Molina, R., Nisbet, L., \& Fernandez, M.L. Examining the Effect of Microteaching Lesson Study Participation on Preservice Teacher Content and Pedagogical Content Knowledge, FCTM Annual Meeting, Oct. 2011. (refereed)

Fernandez, M. L. \& Molina R. Teacher Enacted Curriculum. Algebra Project Meeting, Miami, FL, Jan. 2010.

Fernandez, M. L., Rincon, D., Bowe, G., Molina R., \& Nisbet, L. Investigating AP Dialogues. Algebra Project Meeting, Miami, FL, Jan. 2010.

Fernandez, M. L. \& Roxanne Molina. Written Dialogues as an Instructional Tool in Mathematics. FCTM Annual Meeting, Oct. 2009. (refereed)

PROFESSIONAL HONORS, PRIZES, FELLOWSHIPS

Dissertation Year Fellowship for 2011-2012 awarded by FIU.

\section{OTHER PROFESSIONAL ACTIVITIES}

Reviewer for $10^{\text {th }}$ Annual College of Education and Graduate Student Network Research Conference, 2011.

Reviewer for Association of Mathematics Teacher Education Annual Conference, 2010-2011. 